\title{
New Concepts on Quadripartitioned Bipolar Single Valued Neutrosophic Graph
}

\author{
S. Satham Hussain ${ }^{1}$, G. Muhiuddin ${ }^{2, *}$, N. Durga $^{3}$ and D. Al-Kadi ${ }^{4}$ \\ ${ }^{1}$ PG and Research Department of Mathematics, Jamal Mohamed College (Autonomus), Tiruchirappalli, 620020, India \\ ${ }^{2}$ Department of Mathematics, University of Tabuk, Tabuk, 71491, Saudi Arabia \\ ${ }^{3}$ Division of Mathematics, School of Advanced Sciences, Vellore Institute of Technology-Chennai Campus, Chennai, 600127, India \\ ${ }^{4}$ Department of Mathematics and Statistic, College of Science, Taif University, Taif, 21944, Saudi Arabia \\ *Corresponding Author: G. Muhiuddin. Email: chishtygm@gmail.com
}

Received: 27 April 2021 Accepted: 14 July 2021

\begin{abstract}
The partition of indeterminacy function of the neutrosophic set into the contradiction part and the ignorance part represent the quadripartitioned single valued neutrosophic set. In this work, the new concept of quadripartitioned bipolar single valued neutrosophic graph is established, and the operations on it are studied. The Cartesian product, cross product, lexicographic product, strong product and composition of quadripartitioned bipolar single valued neutrosophic graph are investigated. The proposed concepts are illustrated with examples.
\end{abstract}

\section{KEYWORDS}

Quadripartitioned bipolar single valued neutrosophic graph; operations of quadripartitioned bipolar single valued neutrosophic graph; Cartesian product

\section{Introduction}

Bipolar fuzzy sets are more useful, beneficial, and applicable in real-world situations. It deals with incomplete information since they do not take into account indeterminate or contradictory data, which can be found in a variety of systems [1,2], including belief systems and decisionsupport systems [3,4]. As reality vanishes in a black hole due to Hawking radiation or specific/antiparticular emission, the bipolar domain is the most important condition that persists. In a bipolar fuzzy set, an element with a membership degree of 0 is insignificant to the subsequent property, an element with a membership degree of $(0,1]$ is somewhat satisfied by the property, and an element with a membership degree of $[-1,0]$ is quite satisfied by the implied counter-property. On the other hand, to interpret the degree of true and false membership functions, some restrictions allow only to hold incomplete data, but the handling of indeterminate information remains. Can we see an instance, suppose there are ten patients to check a pandemic during testing. In that time, three patients are having positive, five will have negative, and two are undecided or yet to come. By employing the neutrosophic concepts, it can be expressed as $x(0.3,0.2,0.5)$. For the clear

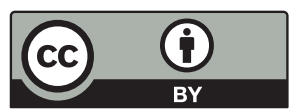

This work is licensed under a Creative Commons Attribution 4.0 International License, which permits unrestricted use, distribution, and reproduction in any medium, provided the original work is properly cited. 
understanding, one can characterize the climate as cold as truth, moderate as indeterminacy and hot as false using a neutrosophic set. Hence the neutrosophic field arises to hold the indeterminacy data as well. It generalizes the aforementioned sets from the philosophical viewpoint. The singlevalued neutrosophic set is the generalization of intuitionistic fuzzy sets and is used expediently to deal with real-world problems, especially in decision support [5]. The computation of belief in that element (truth), they disbelieve in that element (falsehood) and the indeterminacy part of that element with the sum of these three components are strictly less than 1 and it is proposed by Smarandache [6] and references therein.

In the case of neutrosophic sets, indeterminacy function is considered as an individual term and each element $x$ is characterized by a truth-membership function $\mathcal{T}_{A}(x)$, an indeterminacy membership function $\mathcal{I}_{A}(x)$ and a falsity-membership function $\mathcal{F}_{A}(x)$, each of that from the nonstandard unit interval $\left[0^{-}, 1^{+}\right]$. Despite the neutrosophic indeterminacy is independent of the truth and falsity-membership values, but it is more general than the hesitation margin of intuitionistic fuzzy sets. It is not sure whether the indeterminacy values relevant to a particular element correspond to hesitant values about its belonging or not belonging to it. In another way, if a person identifies an indeterminacy membership $\mathcal{I}_{A}(x)$ with a specific event $x$, it becomes difficult to understand whether the person's degree of uncertainty regarding the event's occurrence is $\mathcal{I}_{A}(x)$ or whether the person's degree of uncertainty regarding the event's non-occurrence is $\mathcal{I}_{A}(x)$. As a result, some authors prefer to model the indeterminacy's behaviour in the same way they similar to truth-membership, others may prefer to model it, in the same way, they similar to falsitymembership. Wang et al. [5] initiated the concept of a single-valued neutrosophic set and provide its various properties. It has been widely applied in various fields, such as information fusion in which data are combined from different sensors [7], control theory [8], image processing [9], medical diagnosis [10], decision making [11,12], and graph theory [13-20], etc.

When the indeterminacy portion of the neutrosophic set is divided into two parts, we get four components: 'Contradiction' (both true and false) and 'Unknown' (neither true nor false), that is $\mathcal{T}, \mathcal{C}, \mathcal{U}$ and $\mathcal{F}$ which defines a new set called 'quadripartitioned single valued neutrosophic set', introduced by Chatterjee et al. [21]. This study is completely based on "Belnap's four valued logic" [22] and Smarandache's "Four Numerical valued neutrosophic logic" [23]. By employing the concept of Quadripartitioned Single Valued Neutrosophic Set (QSVNS), this paper presents the quadripartitioned single-valued neutrosophic graphs. Operations on single-valued neutrosophic graphs are studied in [24]. Further, the operations on neutrosophic vague graphs are discussed in [16]. Authors in [25] studied the bipolar quadripartitioned single-valued neutrosophic sets. In $[3,26]$, the authors extensively studied about the concept of bipolar neutrosophic graphs. Moreover, in [27], bipolar single valued neutrosophic graphs are investigated with its related properties. Motivated by the above-mentioned works, to the best of the authors' knowledge, there is no work reported on the concepts of bipolar quadripartioned single-valued neutrosophic graphs with the application. More concepts related to this study have been studied in [28-31]. The major contributions in this work are explained as follows:

1. The notions of Quadripartitioned Bipolar Single Valued Neutrosophic Graphs (QBSVNGs) are introduced. This manuscript makes the first attempt in the literature about the concept in neutrosophic graphs.

2. In addition, the complete and strong QBSVNG are defined. The operations like a Cartesian product, cross product, lexicographic product, strong product and the composition of QBSVNGs with their properties are discussed. 
3. The proposed work will generalise the existing works in the literature $[3,24,25,32]$.

The paper is organized as follows: The basic needed definitions are given in Preliminaries Section 2. The QBSVNGs are introduced and the operations are explained in Section 3 .

\section{Preliminaries}

Definition 2.1 [6] Let $\mathcal{X}$ be a space of points (objects), with generic elements in $\mathcal{X}$ denoted by $x$. A single valued neutrosophic set $\mathcal{A}$ in $\mathcal{X}$ is characterised by truth-membership function $\mathcal{T}_{\mathcal{A}}(\boldsymbol{x})$, indeterminacy-membership function $\mathcal{I}_{\mathcal{A}}(\boldsymbol{x})$ and falsity-membership-function $\mathcal{F}_{\mathcal{A}}(\boldsymbol{x})$.

For each point $x$ in $\mathcal{X}, \mathcal{T}_{\mathcal{A}}(x), \mathcal{I}_{\mathcal{A}}(x), \mathcal{F}_{\mathcal{A}}(x) \in[0,1]$. Also

$\mathcal{A}=\left\{x, \mathcal{T}_{\mathcal{A}}(x), \mathcal{I}_{\mathcal{A}}(x), \mathcal{F}_{\mathcal{A}}(x)\right\}$ and $0 \leq \mathcal{T}_{\mathcal{A}}(x)+\mathcal{I}_{\mathcal{A}}(x)+\mathcal{F}_{\mathcal{A}}(x) \leq 3$

Definition 2.2 [6] A Neutrosophic set $\mathcal{A}$ is contained in another neutrosophic set $\mathcal{B}$, (i.e) $\mathcal{A} \subseteq \mathcal{B}$ if $\forall \mathrm{x} \in \mathcal{X}, \mathcal{T}_{\mathcal{A}}(\mathrm{x}) \leq \mathcal{T}_{\mathcal{B}}(\mathrm{x}), \mathcal{I}_{\mathcal{A}}(\mathrm{x}) \geq \mathcal{I}_{\mathcal{B}}(\mathrm{x})$ and $\mathcal{F}_{\mathcal{A}}(\mathrm{x}) \geq \mathcal{F}_{\mathcal{B}}(\mathrm{x})$

Definition 2.3 [24] A neutrosophic graph is defined as a pair $\mathrm{G}^{*}=(\mathrm{V}, \mathrm{E})$ where

(i) $\mathrm{V}=\left\{\mathrm{v}_{1}, \mathrm{v}_{2}, \ldots, \mathrm{v}_{\mathrm{n}}\right\}$ such that $\mathcal{T}_{\mathcal{A}}: \mathrm{V} \rightarrow[0,1], \mathcal{I}_{\mathcal{A}}: \mathrm{V} \rightarrow[0,1]$ and $\mathcal{F}_{\mathcal{A}}: \mathrm{V} \rightarrow[0,1]$ denote the degrees of truth-membership function, indeterminacy function and falsity-membership function, respectively and

$0 \leq \mathcal{T}_{\mathcal{A}}(\mathrm{v})+\mathcal{I}_{\mathcal{A}}(\mathrm{v})+\mathcal{F}_{\mathcal{A}}(\mathrm{v}) \leq 3, \quad \forall \mathrm{v} \in \mathrm{V}$

(ii) $\mathrm{E} \subseteq \mathrm{V} \times \mathrm{V}$ where $\mathcal{T}_{\mathrm{B}}: \mathrm{E} \rightarrow[0,1], \mathcal{I}_{\mathrm{B}}: \mathrm{E} \rightarrow[0,1]$ and $\mathcal{F}_{\mathrm{B}}: \mathrm{E} \rightarrow[0,1]$ are such that

$\mathcal{T}_{\mathcal{B}}(\mathrm{uv}) \leq \min \left\{\mathrm{T}_{\mathcal{A}}(\mathrm{u}), \mathrm{T}_{\mathcal{A}}(\mathrm{v})\right\}$

$\mathcal{I}_{\mathcal{B}}(\mathrm{uv}) \leq \min \left\{\mathrm{I}_{\mathcal{A}}(\mathrm{u}), \mathrm{I}_{\mathcal{A}}(\mathrm{v})\right\}$,

$\mathcal{F}_{\mathcal{B}}(\mathrm{uv}) \leq \max \left\{\mathrm{F}_{\mathcal{A}}(\mathrm{u}), \mathrm{F}_{\mathcal{A}}(\mathrm{v})\right\}$,

and $0 \leq \mathcal{T}_{\mathcal{B}}(\mathrm{uv})+\mathcal{I}_{\mathcal{B}}(\mathrm{uv})+\mathcal{F}_{\mathcal{B}}(\mathrm{uv}) \leq 3, \quad \forall$ uv $\in$ E.

For more details about the following definitions and results, see the article [21].

Definition 2.4 Let $\mathcal{X}$ be a non-empty set. A quadripartitioned neutrosopohic set (QSVNS) $\mathcal{A}$ over $\mathcal{X}$ characterizes each elements $\mathrm{x}$ in $\mathcal{X}$ by a truth membership function $\mathcal{T}_{\mathcal{A}}$, a contradiction membership function $\mathcal{C}_{\mathcal{A}}$, an ignorance membership function $\mathcal{U}_{\mathcal{A}}$ and a false membership function $\mathcal{F}_{\mathcal{A}}$ such that for each $\mathrm{x} \in \mathcal{X}, \mathcal{T}_{\mathcal{A}}, \mathcal{C}_{\mathcal{A}}, \mathcal{U}_{\mathcal{A}}, \mathcal{F}_{\mathcal{A}} \in[0,1]$ and $0 \leq \mathcal{T}_{\mathcal{A}}(\mathrm{x})+\mathcal{C}_{\mathcal{A}}(\mathrm{x})+\mathcal{U}_{\mathcal{A}}(\mathrm{x})+\mathcal{F}_{\mathcal{A}}(\mathrm{x}) \leq 4$.

Remark 2.5 A QSVNS $\mathfrak{A}$ can be decomposed to yield two SVNS, say, $\mathfrak{A}_{\mathrm{t}}$ and $\mathfrak{A}_{\mathrm{b}}$ where the respective membership functions and these sets are defined as

$\mathcal{T}_{\mathfrak{A}_{\mathrm{t}}}(\mathrm{x})=\mathcal{T}_{\mathfrak{A}}(\mathrm{x})=\mathcal{T}_{\mathfrak{A}_{\mathrm{b}}}(\mathrm{x})$

$\mathcal{I}_{\mathfrak{A}_{\mathrm{t}}}(\mathrm{x})=\mathcal{C}_{\mathfrak{A}}(\mathrm{x}), \mathcal{I}_{\mathfrak{A}_{\mathrm{b}}}(\mathrm{x})=\mathcal{U}_{\mathfrak{A}}(\mathrm{x})$

$\mathcal{F}_{\mathfrak{A}_{\mathrm{t}}}(\mathrm{x})=\mathcal{F}_{\mathfrak{A}}(\mathrm{x})=\mathcal{F}_{\mathfrak{A}_{\mathrm{b}}}(\mathrm{x}), \forall \mathrm{x} \in \mathcal{X}$.

In this respect, we stated that while performing set-theoretic operations over these SVNS, the behaviour of $\mathcal{I}_{\mathfrak{A}_{\mathfrak{t}}}$ is treated similar to that of $\mathcal{T}_{\mathfrak{A}_{\mathrm{t}}}$ while the behaviour of $\mathcal{I}_{\mathfrak{A}_{\mathrm{b}}}$ is modelled in a way similar to that of $\mathcal{F}_{\mathfrak{A}_{\mathrm{b}}}$.

Definition 2.6 A QSVNS is said to be an absolute QSVNS, deoted by $\mathfrak{A}$, if and only if its membership values are respectivley defined as $\mathcal{T}_{\mathfrak{A}}(\mathrm{x})=1, \mathcal{C}_{\mathfrak{A}}(\mathrm{x})=1, \mathcal{U}_{\mathfrak{A}}(\mathrm{x})=0$ and $\mathcal{F}_{\mathfrak{A}}(\mathrm{x})=0$. 
Definition 2.7 Consider two QSVNS $\mathfrak{A}$ and $\mathfrak{B}$ over $\mathcal{X} \cdot \mathfrak{A}$ is said to be contained in $\mathfrak{B}$, denoted by $\mathfrak{A} \subseteq \mathfrak{B}$ if, and only, if $\mathcal{T}_{\mathfrak{A}}(\mathrm{x}) \leq \mathcal{T}_{\mathfrak{B}}(\mathrm{x}), \mathcal{C}_{\mathfrak{A}}(\mathrm{x}) \leq \mathcal{C}_{\mathfrak{B}}(\mathrm{x}), \mathcal{U}_{\mathfrak{A}}(\mathrm{x}) \geq \mathcal{U}_{\mathfrak{B}}(\mathrm{x})$ and $\mathcal{F}_{\mathfrak{A}}(\mathrm{x}) \geq \mathcal{F}_{\mathfrak{B}}(\mathrm{x})$.

Definition 2.8 The complement of a QSVNS $\mathfrak{A}$, is denoted by $\mathfrak{A}^{\mathrm{c}}$ and is defined as $\mathfrak{A}^{\mathrm{c}}=\sum_{\mathrm{i}=1}^{\mathrm{n}}\left\langle\mathcal{F}_{\mathfrak{A}}\left(\mathrm{x}_{\mathrm{i}}\right), \mathcal{U}_{\mathfrak{A}}\left(\mathrm{x}_{\mathrm{i}}\right), \mathcal{C}_{\mathfrak{A}}\left(\mathrm{x}_{\mathrm{i}}\right), \mathcal{T}_{\mathfrak{A}}\left(\mathrm{x}_{\mathrm{i}}\right)\right\rangle, \quad \forall \mathrm{x}_{\mathrm{i}} \in \mathcal{X}$. i.e., $\mathcal{T}_{\mathfrak{A}}^{\mathrm{c}}\left(\mathrm{x}_{\mathrm{i}}\right)=\mathcal{F}_{\mathfrak{A}}\left(\mathrm{x}_{\mathrm{i}}\right), \mathcal{C}_{\mathfrak{A}}^{\mathrm{c}}\left(\mathrm{x}_{\mathrm{i}}\right)=\mathcal{U}_{\mathfrak{A}}\left(\mathrm{x}_{\mathrm{i}}\right), \mathcal{U}_{\mathfrak{A}}^{\mathrm{c}}\left(\mathrm{x}_{\mathrm{i}}\right)=\mathcal{C}_{\mathfrak{A}}\left(\mathrm{x}_{\mathrm{i}}\right), \mathcal{F}_{\mathfrak{A}}^{\mathrm{c}}\left(\mathrm{x}_{\mathrm{i}}\right)=\mathcal{T}_{\mathfrak{A}}\left(\mathrm{x}_{\mathrm{i}}\right), \quad \forall \mathrm{x}_{\mathrm{i}} \in \mathcal{X}$.

Definition 2.9 The union of two QSVNS $\mathfrak{A}$ and $\mathfrak{B}$ is denoted by $\mathfrak{A} \cup \mathfrak{B}$ and is defined as $\mathfrak{A} \cup \mathfrak{B}=\sum_{\mathrm{i}=1}^{\mathrm{n}}\left\langle\mathcal{T}_{\mathfrak{A}}\left(\mathrm{x}_{\mathrm{i}}\right) \vee \mathcal{T}_{\mathfrak{B}}\left(\mathrm{x}_{\mathrm{i}}\right), \mathcal{C}_{\mathfrak{A}}\left(\mathrm{x}_{\mathrm{i}}\right) \vee \mathcal{C}_{\mathfrak{B}}\left(\mathrm{x}_{\mathrm{i}}\right)\right.$ $\left.\mathcal{U}_{\mathfrak{A}}\left(\mathrm{x}_{\mathrm{i}}\right) \wedge \mathcal{U}_{\mathfrak{B}}\left(\mathrm{x}_{\mathrm{i}}\right), \mathcal{F}_{\mathfrak{A}}\left(\mathrm{x}_{\mathrm{i}}\right) \wedge \mathcal{F}_{\mathfrak{B}}\left(\mathrm{x}_{\mathrm{i}}\right)\right\rangle / \mathcal{X}$

Definition 2.10 The intersection of two QSVNS $\mathfrak{A}$ and $\mathfrak{B}$ is denoted by $\mathfrak{A} \cap \mathfrak{B}$ and is defined as

$\mathfrak{A} \cap \mathfrak{B}=\sum_{\mathrm{i}=1}^{\mathrm{n}}\left\langle\mathcal{T}_{\mathfrak{A}}\left(\mathrm{x}_{\mathrm{i}}\right) \wedge \mathcal{T}_{\mathfrak{B}}\left(\mathrm{x}_{\mathrm{i}}\right), \mathcal{C}_{\mathfrak{A}}\left(\mathrm{x}_{\mathrm{i}}\right) \wedge \mathcal{C}_{\mathfrak{B}}\left(\mathrm{x}_{\mathrm{i}}\right)\right.$

$\left.\mathcal{U}_{\mathfrak{A}}\left(\mathrm{x}_{\mathrm{i}}\right) \vee \mathcal{U}_{\mathfrak{B}}\left(\mathrm{x}_{\mathrm{i}}\right), \mathcal{F}_{\mathfrak{A}}\left(\mathrm{x}_{\mathrm{i}}\right) \vee \mathcal{F}_{\mathfrak{B}}\left(\mathrm{x}_{\mathrm{i}}\right)\right\rangle / \mathcal{X}$

Definition 2.11 A quadripartitioned single valued bipolar neutrosophic set (QSVBNS) $\mathfrak{A}$ is $\mathcal{X}$ defined as an object of the form:

$\mathfrak{A}=\left\langle\mathrm{x},(\mathcal{T})_{\mathfrak{A}}^{\mathrm{P}}(\mathrm{x}),(\mathcal{C})_{\mathfrak{A}}^{\mathrm{P}}(\mathrm{x}),(\mathcal{U})_{\mathfrak{A}}^{\mathrm{P}}(\mathrm{x}),(\mathcal{F})_{\mathfrak{A}}^{\mathrm{P}}(\mathrm{x}),(\mathcal{T})_{\mathfrak{A}}^{\mathrm{N}}(\mathrm{x}),(\mathcal{C})_{\mathfrak{A}}^{\mathrm{N}}(\mathrm{x}),(\mathcal{U})_{\mathfrak{A}}^{\mathrm{N}}(\mathrm{x}),(\mathcal{F})_{\mathfrak{A}}^{\mathrm{N}}(\mathrm{x}): \mathrm{x} \in \mathcal{X}\right\rangle$,

where $(\mathcal{T})_{\mathfrak{A}}^{\mathrm{P}},(\mathcal{C})_{\mathfrak{A}}^{\mathrm{P}},(\mathcal{U})_{\mathfrak{A}}^{\mathrm{P}},(\mathcal{F})_{\mathfrak{A}}^{\mathrm{P}}: \mathcal{X} \rightarrow[0,1]$ and $(\mathcal{T})_{\mathfrak{A}}^{\mathrm{N}},(\mathcal{C})_{\mathfrak{A}}^{\mathrm{P}},(\mathcal{U})_{\mathfrak{A}}^{\mathrm{N}},(\mathcal{F})_{\mathfrak{A}}^{\mathrm{N}}: \mathcal{X} \rightarrow[-1,0]$. The positive membership degrees $(\mathcal{T})_{\mathfrak{A}}^{\mathrm{P}}(\mathrm{x}),(\mathcal{C})_{\mathfrak{A}}^{\mathrm{P}}(\mathrm{x}),(\mathcal{U})_{\mathfrak{A}}^{\mathrm{P}}(\mathrm{x}),(\mathcal{F})_{\mathfrak{A}}^{\mathrm{P}}(\mathrm{x})$ denote respectively the truthmembership, a contradiction-membership, an ignorance membership degrees and falsity membership of $\mathrm{x} \in \mathcal{X}$ corresponding to a QSVBNS $\mathfrak{A}$. The negative membership degrees $(\mathcal{T})_{\mathfrak{A}}^{\mathrm{N}}(\mathrm{x}),(\mathcal{C})_{\mathfrak{A}}^{\mathrm{N}}(\mathrm{x}),(\mathcal{U})_{\mathfrak{A}}^{\mathrm{N}}(\mathrm{x}),(\mathcal{F})_{\mathfrak{A}}^{\mathrm{N}}(\mathrm{x})$ denote respectively the truth-membership, a contradiction membership, an ignorance membership and falsity membership of $\mathrm{x} \in \mathcal{X}$ to some explicit counter-property corresponding to a QSVBNS $\mathfrak{A}$.

Definition 2.12 Let $\mathfrak{A}$ and $\mathfrak{B}$ be two QSVBNS over $\mathcal{X}$. Then $\mathfrak{A}$ is said to be included in $\mathfrak{B}$, denoted by $\mathfrak{A} \subseteq \mathfrak{B}$, if for each $\mathrm{x} \in \mathcal{X}$,

$(\mathcal{T})_{\mathfrak{A}}^{\mathrm{P}}(\mathrm{x}) \leq(\mathcal{T})_{\mathfrak{B}}^{\mathrm{P}}(\mathrm{x}),(\mathcal{C})_{\mathfrak{A}}^{\mathrm{P}}(\mathrm{x}) \leq(\mathcal{C})_{\mathfrak{B}}^{\mathrm{P}}(\mathrm{x}),(\mathcal{U})_{\mathfrak{A}}^{\mathrm{P}}(\mathrm{x}) \geq(\mathcal{T})_{\mathfrak{B}}^{\mathrm{P}}(\mathrm{x}),(\mathcal{F})_{\mathfrak{A}}^{\mathrm{P}}(\mathrm{x}) \geq(\mathcal{F})_{\mathfrak{B}}^{\mathrm{P}}(\mathrm{x})$, and $(\mathcal{T})_{\mathfrak{A}}^{\mathrm{N}}(\mathrm{x}) \geq$ $(\mathcal{T})_{\mathfrak{B}}^{\mathrm{N}}(\mathrm{x}),(\mathcal{C})_{\mathfrak{A}}^{\mathrm{N}}(\mathrm{x}) \geq(\mathcal{C})_{\mathfrak{B}}^{\mathrm{N}}(\mathrm{x}),(\mathcal{U})_{\mathfrak{A}}^{\mathrm{N}}(\mathrm{x}) \leq(\mathcal{U})_{\mathfrak{B}}^{\mathrm{N}}(\mathrm{x}),(\mathcal{F})_{\mathfrak{A}}^{\mathrm{N}}(\mathrm{x}) \leq(\mathcal{F})_{\mathfrak{B}}^{\mathrm{N}}(\mathrm{x})$

Definition 2.13 Two QSVBNS $\mathfrak{A}$ and $\mathfrak{B}$ are said to be equal if for each $\mathrm{x} \in \mathcal{X}$,

$(\mathcal{T})_{\mathfrak{A}}^{\mathrm{P}}(\mathrm{x})=(\mathcal{T})_{\mathfrak{B}}^{\mathrm{P}}(\mathrm{x}),(\mathcal{C})_{\mathfrak{A}}^{\mathrm{P}}(\mathrm{x})=(\mathcal{C})_{\mathfrak{B}}^{\mathrm{P}}(\mathrm{x}),(\mathcal{U})_{\mathfrak{A}}^{\mathrm{P}}(\mathrm{x})=(\mathcal{U})_{\mathfrak{B}}^{\mathrm{P}}(\mathrm{x}),(\mathcal{F})_{\mathfrak{A}}^{\mathrm{P}}(\mathrm{x})=(\mathcal{F})_{\mathfrak{B}}^{\mathrm{P}}(\mathrm{x})$, and $(\mathcal{T})_{\mathfrak{A}}^{\mathrm{N}}(\mathrm{x})=$ $(\mathcal{T})_{\mathfrak{B}}^{\mathrm{N}}(\mathrm{x}),(\mathcal{C})_{\mathfrak{A}}^{\mathrm{N}}(\mathrm{x})=(\mathcal{C})_{\mathfrak{B}}^{\mathrm{N}}(\mathrm{x}),(\mathcal{U})_{\mathfrak{A}}^{\mathrm{N}}(\mathrm{x})=(\mathcal{U})_{\mathfrak{B}}^{\mathrm{N}}(\mathrm{x}),(\mathcal{F})_{\mathfrak{A}}^{\mathrm{N}}(\mathrm{x})=(\mathcal{F})_{\mathfrak{B}}^{\mathrm{N}}(\mathrm{x})$

\section{Quadripartitioned Bipolar Single Valued Neutrosophic Graphs}

In this section, the QBSVNGs are introduced, and its operations like Cartesian product, cross product, lexicographic product, strong product and composition are developed.

Definition 3.1 A QBSVNG of a crisp graph $G=(V, E)$ is defined to be a pair $\mathcal{G}=(\mathfrak{R}, \mathfrak{S})$ with $\mathfrak{R}=\left(\mathfrak{R}^{\mathrm{P}}, \mathfrak{R}^{\mathrm{N}}\right)$ and $\mathfrak{S}=\left(\mathfrak{S}^{\mathrm{P}}, \mathfrak{S}^{\mathrm{N}}\right)$, where 
(i) the functions $\left(\mathcal{T}_{\mathfrak{R}}\right)^{\mathrm{P}}: \mathrm{V} \rightarrow[0,1],\left(\mathcal{C}_{\mathfrak{R}}\right)^{\mathrm{P}}: \mathrm{V} \rightarrow[0,1],\left(\mathcal{U}_{\mathfrak{R}}\right)^{\mathrm{P}}: \mathrm{V} \rightarrow[0,1],\left(\mathcal{F}_{\mathfrak{R}}\right)^{\mathrm{P}}: \mathrm{V} \rightarrow[0,1]$ and $\left(\mathcal{T}_{\mathfrak{R}}\right)^{\mathrm{N}}: \mathrm{V} \rightarrow[-1,0],\left(\mathcal{C}_{\mathfrak{R}}\right)^{\mathrm{N}}: \mathrm{V} \rightarrow[-1,0],\left(\mathcal{U}_{\mathfrak{R}}\right)^{\mathrm{N}}: \mathrm{V} \rightarrow[-1,0],\left(\mathcal{F}_{\mathfrak{R}}\right)^{\mathrm{N}}: \mathrm{V} \rightarrow[-1,0]$ represent the degree of truth membership, contradiction membership, ignorance membership and false membership of the element $\mathrm{t} \in \mathrm{V}$, respectively, there is no restriction on the sum $0 \leq\left(\mathcal{T}_{\Re}\right)^{\mathrm{P}}(\mathrm{t})+$ $\left(\mathcal{C}_{\mathfrak{R}}\right)^{\mathrm{P}}(\mathrm{t})+\left(\mathcal{U}_{\mathfrak{R}}\right)^{\mathrm{P}}(\mathrm{t})+\left(\mathcal{F}_{\mathfrak{R}}\right)^{\mathrm{P}}(\mathrm{t}) \leq 4,-4 \leq\left(\mathcal{T}_{\mathfrak{R}}\right)^{\mathrm{N}}(\mathrm{t})+\left(\mathcal{C}_{\mathfrak{R}}\right)^{\mathrm{N}}(\mathrm{t})+\left(\mathcal{U}_{\mathfrak{R}}\right)^{\mathrm{N}}(\mathrm{t})+\left(\mathcal{F}_{\mathfrak{R}}\right)^{\mathrm{N}}(\mathrm{t}) \leq 0$ for all $\mathrm{t} \in \mathrm{V}$.

(ii) the functions $\left(\mathcal{T}_{\mathfrak{S}}\right)^{\mathrm{P}}: \mathrm{E} \subseteq \mathrm{V} \times \mathrm{V} \rightarrow[0,1],\left(\mathcal{C}_{\mathfrak{S}}\right)^{\mathrm{P}}: \mathrm{E} \subseteq \mathrm{V} \times \mathrm{V} \rightarrow[0,1],\left(\mathcal{U}_{\mathfrak{S}}\right)^{\mathrm{P}}: \mathrm{E} \subseteq \mathrm{V} \times$ $\mathrm{V} \rightarrow[0,1],\left(\mathcal{F}_{\mathfrak{S}}\right)^{\mathrm{P}}: \mathrm{E} \subseteq \mathrm{V} \times \mathrm{V} \rightarrow[0,1]$ and $\left(\mathcal{T}_{\mathfrak{S}}\right)^{\mathrm{N}}: \mathrm{E} \subseteq \mathrm{V} \times \mathrm{V} \rightarrow[-1,0],\left(\mathcal{C}_{\mathfrak{S}}\right)^{\mathrm{N}}: \mathrm{E} \subseteq \mathrm{V} \times \mathrm{V} \rightarrow$ $[-1,0],\left(\mathcal{U}_{\mathfrak{S}}\right)^{\mathrm{N}}: \mathrm{E} \subseteq \mathrm{V} \times \mathrm{V} \rightarrow[-1,0],\left(\mathcal{F}_{\mathfrak{S}}\right)^{\mathrm{N}}: \mathrm{E} \subseteq \mathrm{V} \times \mathrm{V} \rightarrow[-1,0]$ are defined as

$\left(\mathcal{T}_{\mathfrak{S}}\right)^{\mathrm{P}}(\mathrm{ts}) \leq \min \left\{\left(\mathcal{T}_{\mathfrak{R}}\right)^{\mathrm{P}}(\mathrm{t}),\left(\mathcal{T}_{\mathfrak{R}}\right)^{\mathrm{P}}(\mathrm{s})\right\}$

$\left(\mathcal{C}_{\mathfrak{S}}\right)^{\mathrm{P}}(\mathrm{ts}) \leq \min \left\{\left(\mathcal{C}_{\mathfrak{R}}\right)^{\mathrm{P}}(\mathrm{t}),\left(\mathcal{C}_{\mathfrak{R}}\right)^{\mathrm{P}}(\mathrm{s})\right\}$

$\left(\mathcal{U}_{\mathfrak{S}}\right)^{\mathrm{P}}(\mathrm{ts}) \leq \max \left\{\left(\mathcal{U}_{\mathfrak{R}}\right)^{\mathrm{P}}(\mathrm{t}),\left(\mathcal{U}_{\mathfrak{R}}\right)^{\mathrm{P}}(\mathrm{s})\right\}$

$\left(\mathcal{F}_{\mathfrak{S}}\right)^{\mathrm{P}}(\mathrm{ts}) \leq \max \left\{\left(\mathcal{F}_{\mathfrak{R}}\right)^{\mathrm{P}}(\mathrm{t}),\left(\mathcal{F}_{\mathfrak{R}}\right)^{\mathrm{P}}(\mathrm{s})\right\}, \quad \forall$ ts $\in \mathrm{E}$,

$\left(\mathcal{T}_{\mathfrak{S}}\right)^{\mathrm{N}}(\mathrm{ts}) \geq \max \left\{\left(\mathcal{T}_{\mathfrak{R}}\right)^{\mathrm{N}}(\mathrm{t}),\left(\mathcal{T}_{\mathfrak{R}}\right)^{\mathrm{N}}(\mathrm{s})\right\}$

$\left(\mathcal{C}_{\mathfrak{S}}\right)^{\mathrm{N}}(\mathrm{ts}) \geq \max \left\{\left(\mathcal{C}_{\mathfrak{R}}\right)^{\mathrm{N}}(\mathrm{t}),\left(\mathcal{C}_{\mathfrak{R}}\right)^{\mathrm{N}}(\mathrm{s})\right\}$

$\left(\mathcal{U}_{\mathfrak{S}}\right)^{\mathrm{N}}(\mathrm{ts}) \geq \min \left\{\left(\mathcal{U}_{\mathfrak{R}}\right)^{\mathrm{N}}(\mathrm{t}),\left(\mathcal{U}_{\mathfrak{R}}\right)^{\mathrm{N}}(\mathrm{s})\right\}$

$\left(\mathcal{F}_{\mathfrak{S}}\right)^{\mathrm{N}}(\mathrm{ts}) \geq \min \left\{\left(\mathcal{F}_{\mathfrak{R}}\right)^{\mathrm{N}}(\mathrm{t}),\left(\mathcal{F}_{\mathfrak{R}}\right)^{\mathrm{N}}(\mathrm{s})\right\}, \quad \forall$ ts $\in \mathrm{E}$.

There is no restriction on the sum of $0 \leq\left(\mathcal{T}_{\mathfrak{S}}\right)^{\mathrm{P}}(\mathrm{ts})+\left(\mathcal{C}_{\mathfrak{S}}\right)^{\mathrm{P}}(\mathrm{ts})+\left(\mathcal{U}_{\mathfrak{S}}\right)^{\mathrm{P}}(\mathrm{ts})+\left(\mathcal{F}_{\mathfrak{S}}\right)^{\mathrm{P}}(\mathrm{ts}) \leq 4$, $-4 \leq\left(\mathcal{T}_{\mathfrak{S}}\right)^{\mathrm{N}}(\mathrm{ts})+\left(\mathcal{C}_{\mathfrak{S}}\right)^{\mathrm{N}}(\mathrm{ts})+\left(\mathcal{U}_{\mathfrak{S}}\right)^{\mathrm{N}}(\mathrm{ts})+\left(\mathcal{F}_{\mathfrak{S}}\right)^{\mathrm{N}}(\mathrm{ts}) \leq 0$ for all $\mathrm{t} \in \mathrm{V}$, for all ts $\in$ E. Here $\mathfrak{R}$ is the QBSVN vertex set of $\mathcal{G}$ and $\mathfrak{S}$ is the QBSVN edge set of $\mathcal{G}$.

Example 3.1 Consider a crisp graph $\mathrm{G}=(\mathrm{V}, \mathrm{E})$ such that $\mathrm{V}=\{\mathrm{a}, \mathrm{b}, \mathrm{c}\}$ and $\mathrm{E}=\{\mathrm{ab}, \mathrm{bc}, \mathrm{ca}\}$ the corresponding QBSVNG $\mathcal{G}=(\mathfrak{R}, \mathfrak{S})$ is shown in Fig. 1 .

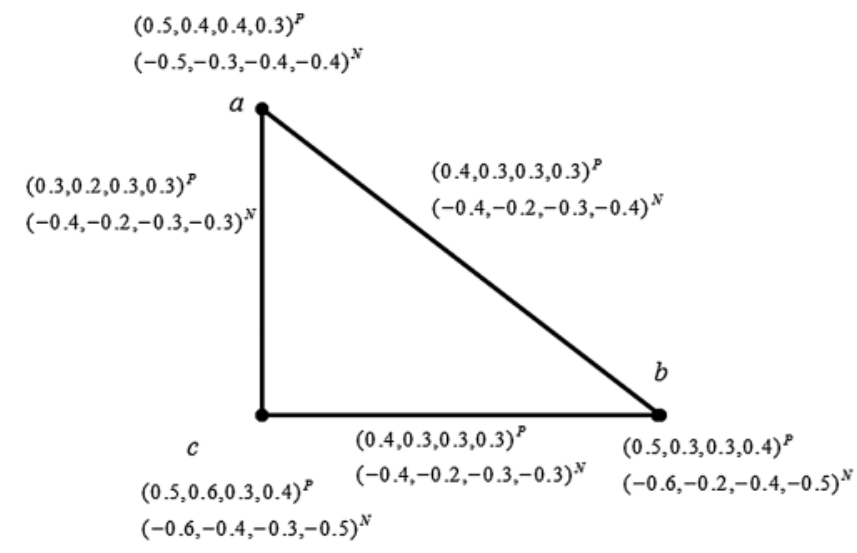

Figure 1: Quadripartitioned bipolar single valued neutrosophic graph 
Definition 3.2 A QBSVNG $\mathcal{G}=(\mathfrak{R}, \mathfrak{S})$ is called complete if the following conditions are satisfied

$$
\begin{aligned}
& \left(\mathcal{T}_{\mathfrak{S}}\right)^{\mathrm{P}}(\mathrm{ts})=\min \left\{\left(\mathcal{T}_{\mathfrak{R}}\right)^{\mathrm{P}}(\mathrm{t}),\left(\mathcal{T}_{\mathfrak{R}}\right)^{\mathrm{P}}(\mathrm{s})\right\} \\
& \left(\mathcal{C}_{\mathfrak{S}}\right)^{\mathrm{P}}(\mathrm{ts})=\min \left\{\left(\mathcal{C}_{\mathfrak{R}}\right)^{\mathrm{P}}(\mathrm{t}),\left(\mathcal{C}_{\mathfrak{R}}\right)^{\mathrm{P}}(\mathrm{s})\right\} \\
& \left(\mathcal{U}_{\mathfrak{S}}\right)^{\mathrm{P}}(\mathrm{ts})=\max \left\{\left(\mathcal{U}_{\mathfrak{R}}\right)^{\mathrm{P}}(\mathrm{t}),\left(\mathcal{U}_{\mathfrak{R}}\right)^{\mathrm{P}}(\mathrm{s})\right\} \\
& \left(\mathcal{F}_{\mathfrak{S}}\right)^{\mathrm{P}}(\mathrm{ts})=\max \left\{\left(\mathcal{F}_{\mathfrak{R}}\right)^{\mathrm{P}}(\mathrm{t}),\left(\mathcal{F}_{\mathfrak{R}}\right)^{\mathrm{P}}(\mathrm{s})\right\}, \quad \forall \mathrm{t}, \mathrm{s} \in \mathrm{V} \text { and } \\
& \left(\mathcal{T}_{\mathfrak{S}}\right)^{\mathrm{N}}(\mathrm{ts})=\max \left\{\left(\mathcal{T}_{\mathfrak{R}}\right)^{\mathrm{N}}(\mathrm{t}),\left(\mathcal{T}_{\mathfrak{R}}\right)^{\mathrm{N}}(\mathrm{s})\right\} \\
& \left(\mathcal{C}_{\mathfrak{S}}\right)^{\mathrm{N}}(\mathrm{ts})=\max \left\{\left(\mathcal{C}_{\mathfrak{R}}\right)^{\mathrm{N}}(\mathrm{t}),\left(\mathcal{C}_{\mathfrak{R}}\right)^{\mathrm{N}}(\mathrm{s})\right\} \\
& \left(\mathcal{U}_{\mathfrak{S}}\right)^{\mathrm{N}}(\mathrm{ts})=\min \left\{\left(\mathcal{U}_{\mathfrak{R}}\right)^{\mathrm{N}}(\mathrm{t}),\left(\mathcal{U}_{\mathfrak{R}}\right)^{\mathrm{N}}(\mathrm{s})\right\} \quad \\
& \left(\mathcal{F}_{\mathfrak{S}}\right)^{\mathrm{N}}(\mathrm{ts})=\min \left\{\left(\mathcal{F}_{\mathfrak{R}}\right)^{\mathrm{N}}(\mathrm{t}),\left(\mathcal{F}_{\mathfrak{R}}\right)^{\mathrm{N}}(\mathrm{s})\right\}, \quad \forall \mathrm{t}, \mathrm{s} \in \mathrm{V} .
\end{aligned}
$$

Example 3.2 Consider a crisp graph $\mathrm{G}=(\mathrm{V}, \mathrm{E})$ such that $\mathrm{V}=\{\mathrm{a}, \mathrm{b}, \mathrm{c}\}$ and $\mathrm{E}=\{\mathrm{ab}, \mathrm{bc}, \mathrm{ca}\}$ the corresponding QBSVNG $\mathcal{G}=(\mathfrak{R}, \mathfrak{S})$ is shown in Fig. 2.

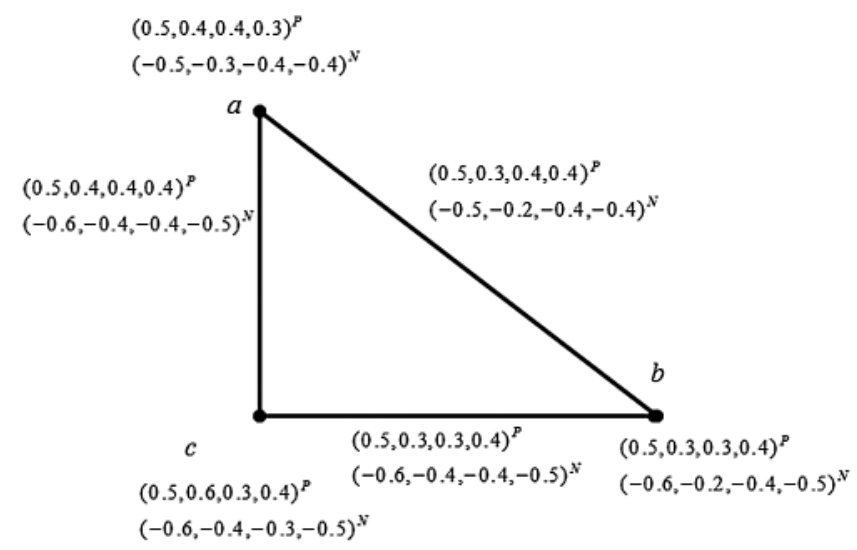

Figure 2: Complete bipolar quadripatitioned single valued neutrosophic graph

Definition 3.3 A QBSVNG $\mathcal{G}=(\mathfrak{R}, \mathfrak{S})$ is called strong if the following conditions are satisfied:

$$
\begin{aligned}
& \left(\mathcal{T}_{\mathfrak{S}}\right)^{\mathrm{P}}(\mathrm{ts})=\min \left\{\left(\mathcal{T}_{\mathfrak{R}}\right)^{\mathrm{P}}(\mathrm{t}),\left(\mathcal{T}_{\mathfrak{R}}\right)^{\mathrm{P}}(\mathrm{s})\right\} \\
& \left(\mathcal{C}_{\mathfrak{S}}\right)^{\mathrm{P}}(\mathrm{ts})=\min \left\{\left(\mathcal{C}_{\mathfrak{R}}\right)^{\mathrm{P}}(\mathrm{t}),\left(\mathcal{C}_{\mathfrak{R}}\right)^{\mathrm{P}}(\mathrm{s})\right\} \\
& \left(\mathcal{U}_{\mathfrak{S}}\right)^{\mathrm{P}}(\mathrm{ts})=\max \left\{\left(\mathcal{U}_{\mathfrak{R}}\right)^{\mathrm{P}}(\mathrm{t}),\left(\mathcal{U}_{\mathfrak{R}}\right)^{\mathrm{P}}(\mathrm{s})\right\} \\
& \left(\mathcal{F}_{\mathfrak{S}}\right)^{\mathrm{P}}(\mathrm{ts})=\max \left\{\left(\mathcal{F}_{\mathfrak{R}}\right)^{\mathrm{P}}(\mathrm{t}),\left(\mathcal{F}_{\mathfrak{R}}\right)^{\mathrm{P}}(\mathrm{s})\right\}, \quad \forall \text { ts } \in \mathrm{E} \text { and } \\
& \left(\mathcal{T}_{\mathfrak{S}}\right)^{\mathrm{N}}(\mathrm{ts})=\max \left\{\left(\mathcal{T}_{\mathfrak{R}}\right)^{\mathrm{N}}(\mathrm{t}),\left(\mathcal{T}_{\mathfrak{R}}\right)^{\mathrm{N}}(\mathrm{s})\right\} \\
& \left(\mathcal{C}_{\mathfrak{S}}\right)^{\mathrm{N}}(\mathrm{ts})=\max \left\{\left(\mathcal{C}_{\mathfrak{R}}\right)^{\mathrm{N}}(\mathrm{t}),\left(\mathcal{C}_{\mathfrak{R}}\right)^{\mathrm{N}}(\mathrm{s})\right\} \\
& \left(\mathcal{U}_{\mathfrak{S}}\right)^{\mathrm{N}}(\mathrm{ts})=\min \left\{\left(\mathcal{U}_{\mathfrak{R}}\right)^{\mathrm{N}}(\mathrm{t}),\left(\mathcal{U}_{\mathfrak{R}}\right)^{\mathrm{N}}(\mathrm{s})\right\}
\end{aligned}
$$


$\left(\mathcal{F}_{\mathfrak{S}}\right)^{\mathrm{N}}(\mathrm{ts})=\min \left\{\left(\mathcal{F}_{\mathfrak{R}}\right)^{\mathrm{N}}(\mathrm{t}),\left(\mathcal{F}_{\mathfrak{R}}\right)^{\mathrm{N}}(\mathrm{s})\right\}, \quad \forall$ ts $\in \mathrm{E}$.

Definition 3.4 The Cartesian product of two QBSVNGs, $\mathcal{G}_{1}$ and $\mathcal{G}_{2}$ is denoted by the pair $\mathcal{G}_{1} \times \mathcal{G}_{2}=\left(\mathfrak{R}_{1} \times \mathfrak{R}_{2}, \mathfrak{S}_{1} \times \mathfrak{S}_{2}\right)$ and defined as

(i) $(\mathcal{T})_{\left(\Re_{1} \times \Re_{2}\right)}^{\mathrm{P}}(\mathrm{kl})=(\mathcal{T})_{\mathfrak{R}_{1}}^{\mathrm{P}}(\mathrm{k}) \wedge(\mathcal{T})_{\mathfrak{R}_{2}}^{\mathrm{P}}(\mathrm{l})$

$(\mathcal{C})_{\left(\Re_{1} \times \Re_{2}\right)}^{\mathrm{P}}(\mathrm{kl})=(\mathcal{C})_{\mathfrak{R}_{1}}^{\mathrm{P}}(\mathrm{k}) \wedge(\mathcal{C})_{\mathfrak{R}_{2}}^{\mathrm{P}}(\mathrm{l})$

$(\mathcal{U})_{\left(\Re_{1} \times \Re_{2}\right)}^{\mathrm{P}}(\mathrm{kl})=(\mathcal{U})_{\mathfrak{R}_{1}}^{\mathrm{P}}(\mathrm{k}) \vee(\mathcal{U})_{\mathfrak{R}_{2}}^{\mathrm{P}}(\mathrm{l})$

$(\mathcal{F})_{\left(\Re_{1} \times \mathfrak{R}_{2}\right)}^{\mathrm{P}}(\mathrm{kl})=(\mathcal{F})_{\mathfrak{R}_{1}}^{\mathrm{P}}(\mathrm{k}) \vee(\mathcal{F})_{\mathfrak{R}_{2}}^{\mathrm{P}}(\mathrm{l})$

$(\mathcal{T})_{\left(\Re_{1} \times \Re_{2}\right)}^{\mathrm{N}}(\mathrm{kl})=(\mathcal{T})_{\mathfrak{R}_{1}}^{\mathrm{N}}(\mathrm{k}) \vee(\mathcal{T})_{\mathfrak{R}_{2}}^{\mathrm{N}}(\mathrm{l})$

$(\mathcal{C})_{\left(\Re_{1} \times \Re_{2}\right)}^{\mathrm{N}}(\mathrm{kl})=(\mathcal{C})_{\mathfrak{R}_{1}}^{\mathrm{N}}(\mathrm{k}) \vee(\mathcal{C})_{\mathfrak{R}_{2}}^{\mathrm{N}}(\mathrm{l})$

$(\mathcal{U})_{\left(\Re_{1} \times \Re_{2}\right)}^{\mathrm{N}}(\mathrm{kl})=(\mathcal{U})_{\mathfrak{R}_{1}}^{\mathrm{N}}(\mathrm{k}) \wedge(\mathcal{U})_{\mathfrak{R}_{2}}^{\mathrm{N}}(\mathrm{l})$

$(\mathcal{F})_{\left(\Re_{1} \times \Re_{2}\right)}^{\mathrm{N}}(\mathrm{kl})=(\mathcal{F})_{\mathfrak{R}_{1}}^{\mathrm{N}}(\mathrm{k}) \wedge(\mathcal{F})_{\mathfrak{R}_{2}}^{\mathrm{N}}(\mathrm{l})$

for all $(\mathrm{k}, 1) \in \mathfrak{R}_{1} \times \mathfrak{R}_{2}$.

The membership value of the edges in $\mathcal{G}_{1} \times \mathcal{G}_{2}$ can be calculated as

(ii) $(\mathcal{T})_{\left(\mathfrak{S}_{1} \times \mathfrak{S}_{2}\right)}^{\mathrm{P}}\left(\mathrm{kl}_{1}\right)\left(\mathrm{kl}_{2}\right)=(\mathcal{T})_{\mathfrak{R}_{1}}^{\mathrm{P}}(\mathrm{k}) \wedge(\mathcal{T})_{\mathfrak{S}_{2}}^{\mathrm{P}}\left(\mathrm{l}_{1} \mathrm{l}_{2}\right)$

$(\mathcal{C})_{\left(\mathfrak{S}_{1} \times \mathfrak{S}_{2}\right)}^{\mathrm{P}}\left(\mathrm{kl}_{1}\right)\left(\mathrm{kl}_{2}\right)=(\mathcal{C})_{\mathfrak{R}_{1}}^{\mathrm{P}}(\mathrm{k}) \wedge(\mathcal{C})_{\mathfrak{S}_{2}}^{\mathrm{P}}\left(l_{1} \mathrm{l}_{2}\right)$

$(\mathcal{U})_{\left(\mathfrak{S}_{1} \times \mathfrak{S}_{2}\right)}^{\mathrm{P}}\left(\mathrm{kl}_{1}\right)\left(\mathrm{kl}_{2}\right)=(\mathcal{U})_{\mathfrak{R}_{1}}^{\mathrm{P}}(\mathrm{k}) \vee(\mathcal{U})_{\mathfrak{S}_{2}}^{\mathrm{P}}\left(l_{1} \mathrm{l}_{2}\right)$

$(\mathcal{F})_{\left(\mathfrak{S}_{1} \times \mathfrak{S}_{2}\right)}^{\mathrm{P}}\left(\mathrm{kl}_{1}\right)\left(\mathrm{kl}_{2}\right)=(\mathcal{F})_{\mathfrak{R}_{1}}^{\mathrm{P}}(\mathrm{k}) \vee(\mathcal{F})_{\mathfrak{S}_{2}}^{\mathrm{P}}\left(1_{1} \mathrm{l}_{2}\right)$

$(\mathcal{T})_{\left(\mathfrak{S}_{1} \times \mathfrak{S}_{2}\right)}^{\mathrm{N}}\left(\mathrm{kl}_{1}\right)\left(\mathrm{kl}_{2}\right)=(\mathcal{T})_{\mathfrak{R}_{1}}^{\mathrm{N}}(\mathrm{k}) \vee(\mathcal{T})_{\mathfrak{S}_{2}}^{\mathrm{N}}\left(1_{1} \mathrm{l}_{2}\right)$

$(\mathcal{C})_{\left(\mathfrak{S}_{1} \times \mathfrak{S}_{2}\right)}^{\mathrm{N}}\left(\mathrm{kl}_{1}\right)\left(\mathrm{kl}_{2}\right)=(\mathcal{C})_{\mathfrak{R}_{1}}^{\mathrm{N}}(\mathrm{k}) \vee(\mathcal{C})_{\mathfrak{S}_{2}}^{\mathrm{N}}\left(l_{1} \mathrm{l}_{2}\right)$

$(\mathcal{U})_{\left(\mathfrak{S}_{1} \times \mathfrak{S}_{2}\right)}^{\mathrm{N}}\left(\mathrm{kl}_{1}\right)\left(\mathrm{kl}_{2}\right)=(\mathcal{U})_{\mathfrak{R}_{1}}^{\mathrm{N}}(\mathrm{k}) \wedge(\mathcal{U})_{\mathfrak{S}_{2}}^{\mathrm{N}}\left(1_{1} l_{2}\right)$

$(\mathcal{F})_{\left(\mathfrak{S}_{1} \times \mathfrak{S}_{2}\right)}^{\mathrm{N}}\left(\mathrm{kl}_{1}\right)\left(\mathrm{kl}_{2}\right)=(\mathcal{F})_{\mathfrak{R}_{1}}^{\mathrm{N}}(\mathrm{k}) \wedge(\mathcal{F})_{\mathfrak{S}_{2}}^{\mathrm{N}}\left(1_{1} \mathrm{l}_{2}\right)$

for all $\mathrm{k} \in \mathfrak{R}_{1}, 1_{1} 1_{2} \in \mathfrak{S}_{2}$.

(iii) $(\mathcal{T})_{\left(\mathfrak{S}_{1} \times \mathfrak{S}_{2}\right)}^{\mathrm{P}}\left(\mathrm{k}_{1} \mathrm{l}\right)\left(\mathrm{k}_{2} \mathrm{l}\right)=(\mathcal{T})_{\mathfrak{R}_{2}}^{\mathrm{P}}(\mathrm{l}) \wedge(\mathcal{T})_{\mathfrak{S}_{1}}^{\mathrm{P}}\left(\mathrm{k}_{1} \mathrm{k}_{2}\right)$

$(\mathcal{C})_{\left(\mathfrak{S}_{1} \times \mathfrak{S}_{2}\right)}^{\mathrm{P}}\left(\mathrm{k}_{1} \mathrm{l}\right)\left(\mathrm{k}_{2} \mathrm{l}\right)=(\mathcal{C})_{\mathfrak{R}_{2}}^{\mathrm{P}}(\mathrm{l}) \wedge(\mathcal{C})_{\mathfrak{S}_{1}}^{\mathrm{P}}\left(\mathrm{k}_{1} \mathrm{k}_{2}\right)$

$(\mathcal{U})_{\left(\mathfrak{S}_{1} \times \mathfrak{S}_{2}\right)}^{\mathrm{P}}\left(\mathrm{k}_{1} \mathrm{l}\right)\left(\mathrm{k}_{2} \mathrm{l}\right)=(\mathcal{U})_{\mathfrak{R}_{2}}^{\mathrm{P}}(\mathrm{l}) \vee(\mathcal{U})_{\mathfrak{S}_{1}}^{\mathrm{P}}\left(\mathrm{k}_{1} \mathrm{k}_{2}\right)$

$(\mathcal{F})_{\left(\mathfrak{S}_{1} \times \mathfrak{S}_{2}\right)}^{\mathrm{P}}\left(\mathrm{k}_{1} \mathrm{l}\right)\left(\mathrm{k}_{2} \mathrm{l}\right)=(\mathcal{F})_{\mathfrak{R}_{2}}^{\mathrm{P}}(\mathrm{l}) \vee(\mathcal{F})_{\mathfrak{S}_{1}}^{\mathrm{P}}\left(\mathrm{k}_{1} \mathrm{k}_{2}\right)$

$(\mathcal{T})_{\left(\mathfrak{S}_{1} \times \mathfrak{S}_{2}\right)}^{\mathrm{N}}\left(\mathrm{k}_{1} \mathrm{l}\right)\left(\mathrm{k}_{2} \mathrm{l}\right)=(\mathcal{T})_{\mathfrak{R}_{2}}^{\mathrm{N}}(\mathrm{l}) \vee(\mathcal{T})_{\mathfrak{S}_{1}}^{\mathrm{N}}\left(\mathrm{k}_{1} \mathrm{k}_{2}\right)$

$(\mathcal{C})_{\left(\mathfrak{S}_{1} \times \mathfrak{S}_{2}\right)}^{\mathrm{N}}\left(\mathrm{k}_{1} \mathrm{l}\right)\left(\mathrm{k}_{2} \mathrm{l}\right)=(\mathcal{C})_{\mathfrak{R}_{2}}^{\mathrm{N}}(\mathrm{l}) \vee(\mathcal{C})_{\mathfrak{S}_{1}}^{\mathrm{N}}\left(\mathrm{k}_{1} \mathrm{k}_{2}\right)$ 
$(\mathcal{U})_{\left(\mathfrak{S}_{1} \times \mathfrak{S}_{2}\right)}^{\mathrm{N}}\left(\mathrm{k}_{1} \mathrm{l}\right)\left(\mathrm{k}_{2} \mathrm{l}\right)=(\mathcal{U})_{\mathfrak{R}_{2}}^{\mathrm{N}}(\mathrm{l}) \wedge(\mathcal{U})_{\mathfrak{S}_{1}}^{\mathrm{N}}\left(\mathrm{k}_{1} \mathrm{k}_{2}\right)$

$(\mathcal{F})_{\left(\mathfrak{S}_{1} \times \mathfrak{S}_{2}\right)}^{\mathrm{N}}\left(\mathrm{k}_{1} \mathrm{l}\right)\left(\mathrm{k}_{2} \mathrm{l}\right)=(\mathcal{F})_{\mathfrak{R}_{2}}^{\mathrm{N}}(\mathrm{l}) \wedge(\mathcal{F})_{\mathfrak{S}_{1}}^{\mathrm{N}}\left(\mathrm{k}_{1} \mathrm{k}_{2}\right)$

for all $1 \in \mathfrak{R}_{2}, \mathrm{k}_{1} \mathrm{k}_{2} \in \mathfrak{S}_{1}$.

Example 3.3 Consider two QBSVNG $\mathcal{G}_{1}$ and $\mathcal{G}_{2}$ as shown in Fig. 3. Then Cartesian product of $\mathcal{G}_{1} \times \mathcal{G}_{2}$ is shown in Fig. 4 .
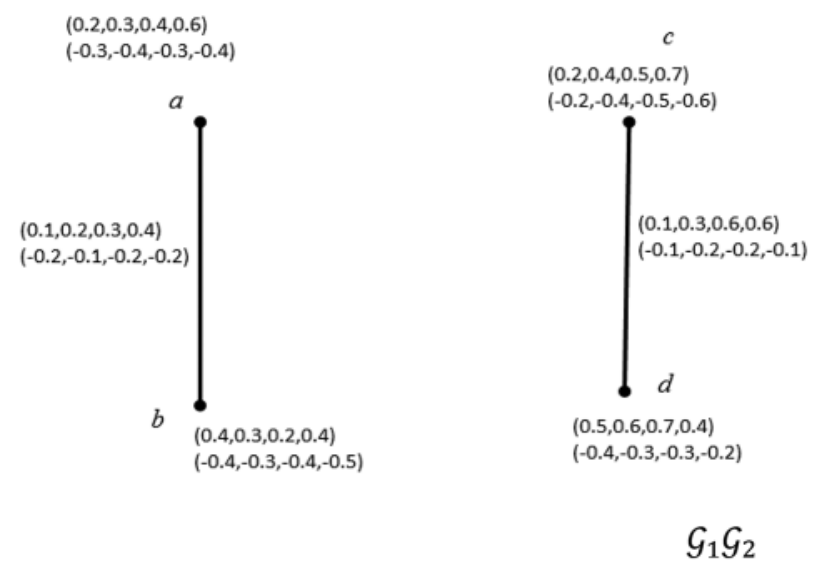

Figure 3: $\mathcal{G}_{1}$ and $\mathcal{G}_{1}$ Two bipolar quadripatitioned single valued neutrosophic graph

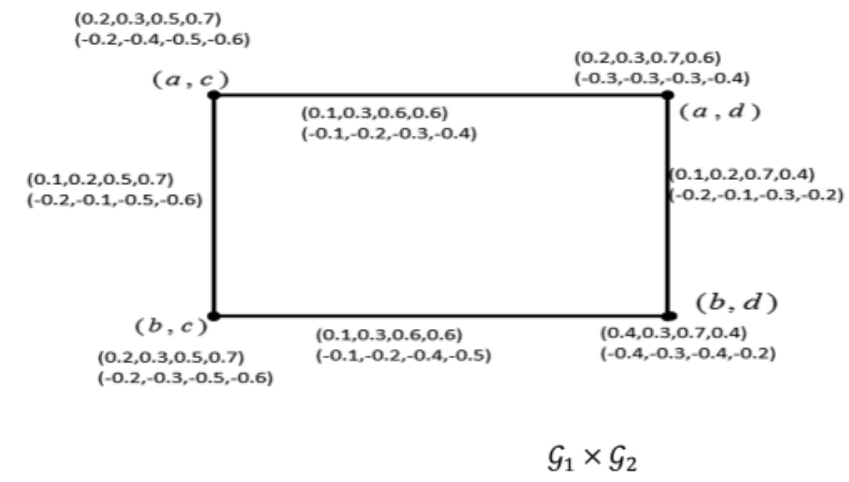

Figure 4: Cartesian product of quadripatitioned single valued neutrosophic graph

Proposition 3.5 The Cartesian product $\mathcal{G}_{1} \times \mathcal{G}_{2}=\left(\mathfrak{R}_{1} \times \mathfrak{R}_{2}, \mathfrak{S}_{1} \times \mathfrak{S}_{2}\right)$ of two QBSVNGs $\mathcal{G}_{1}$ and $\mathcal{G}_{2}$ is also the QBSVNG of $\mathcal{G}_{1} \times \mathcal{G}_{2}$.

Proof. We consider two cases.

Case 1: for $\mathrm{k} \in \mathfrak{R}_{1}, 1_{1} \mathrm{l}_{2} \in \mathfrak{S}_{2}$,

$(\mathcal{T})_{\left(\mathfrak{S}_{1} \times \mathfrak{S}_{2}\right)}^{\mathrm{P}}\left(\left(\mathrm{kl}_{1}\right)\left(\mathrm{kl}_{2}\right)\right)=(\mathcal{T})_{\mathfrak{R}_{1}}^{\mathrm{P}}(\mathrm{k}) \wedge(\mathcal{T})_{\mathfrak{S}_{2}}^{\mathrm{P}}\left(\mathrm{l}_{1} \mathrm{l}_{2}\right)$

$\leq(\mathcal{T})_{\mathfrak{R}_{1}}^{\mathrm{P}}(\mathrm{k}) \wedge\left[(\mathcal{T})_{\mathfrak{R}_{2}}^{\mathrm{P}}\left(l_{1}\right) \wedge(\mathcal{T})_{\mathfrak{R}_{2}}^{\mathrm{P}}\left(l_{2}\right)\right]$ 


$$
\begin{aligned}
& =\left[(\mathcal{T})_{\mathfrak{R}_{1}}^{\mathrm{P}}(\mathrm{k}) \wedge(\mathcal{T})_{\mathfrak{R}_{2}}^{\mathrm{P}}\left(\mathrm{l}_{1}\right)\right] \wedge\left[(\mathcal{T})_{\mathfrak{R}_{1}}^{\mathrm{P}}(\mathrm{k}) \wedge(\mathcal{T})_{\mathfrak{R}_{2}}^{\mathrm{P}}\left(\mathrm{l}_{2}\right)\right] \\
& =(\mathcal{T})_{\left(\Re_{1} \times \Re_{2}\right)}^{\mathrm{P}}\left(\mathrm{k}, 1_{1}\right) \wedge(\mathcal{T})_{\left(\Re_{1} \times \Re_{2}\right)}^{\mathrm{P}}\left(\mathrm{k}, 1_{2}\right) \\
& (\mathcal{C})_{\left(\mathfrak{S}_{1} \times \mathfrak{S}_{2}\right)}^{\mathrm{P}}\left(\left(\mathrm{kl}_{1}\right)\left(\mathrm{kl}_{2}\right)\right)=(\mathcal{C})_{\mathfrak{R}_{1}}^{\mathrm{P}}(\mathrm{k}) \wedge(\mathcal{C})_{\mathfrak{S}_{2}}^{\mathrm{P}}\left(1_{1} \mathrm{l}_{2}\right) \\
& \leq(\mathcal{C})_{\mathfrak{R}_{1}}^{\mathrm{P}}(\mathrm{k}) \wedge\left[(\mathcal{C})_{\mathfrak{R}_{2}}^{\mathrm{P}}\left(\mathrm{l}_{1}\right) \wedge(\mathcal{C})_{\mathfrak{R}_{2}}^{\mathrm{P}}\left(\mathrm{l}_{2}\right)\right] \\
& =\left[(\mathcal{C})_{\mathfrak{R}_{1}}^{\mathrm{P}}(\mathrm{k}) \wedge(\mathcal{C})_{\mathfrak{R}_{2}}^{\mathrm{P}}\left(1_{1}\right)\right] \wedge\left[(\mathcal{C})_{\mathfrak{R}_{1}}^{\mathrm{P}}(\mathrm{k}) \wedge(\mathcal{C})_{\mathfrak{R}_{2}}^{\mathrm{P}}\left(\mathrm{l}_{2}\right)\right] \\
& =(\mathcal{C})_{\left(\Re_{1} \times \Re_{2}\right)}^{\mathrm{P}}\left(\mathrm{k}, 1_{1}\right) \wedge(\mathcal{C})_{\left(\Re_{1} \times \mathfrak{R}_{2}\right)}^{\mathrm{P}}\left(\mathrm{k}, 1_{2}\right) \\
& (\mathcal{U})_{\left(\mathfrak{S}_{1} \times \mathfrak{S}_{2}\right)}^{\mathrm{P}}\left(\left(\mathrm{kl}_{1}\right)\left(\mathrm{kl}_{2}\right)\right)=(\mathcal{U})_{\mathfrak{R}_{1}}^{\mathrm{P}}(\mathrm{k}) \vee(\mathcal{U})_{\mathfrak{S}_{2}}^{\mathrm{P}}\left(l_{1} \mathrm{l}_{2}\right) \\
& \leq(\mathcal{U})_{\mathfrak{R}_{1}}^{\mathrm{P}}(\mathrm{k}) \vee\left[(\mathcal{U})_{\mathfrak{R}_{2}}^{\mathrm{P}}\left(l_{1}\right) \vee(\mathcal{U})_{\mathfrak{R}_{2}}^{\mathrm{P}}\left(l_{2}\right)\right] \\
& =\left[(\mathcal{U})_{\mathfrak{R}_{1}}^{\mathrm{P}}(\mathrm{k}) \vee(\mathcal{U})_{\mathfrak{R}_{2}}^{\mathrm{P}}\left(\mathrm{l}_{1}\right)\right] \vee\left[(\mathcal{U})_{\mathfrak{R}_{1}}^{\mathrm{P}}(\mathrm{k}) \vee(\mathcal{U})_{\mathfrak{R}_{2}}^{\mathrm{P}}\left(l_{2}\right)\right] \\
& =(\mathcal{U})_{\left(\Re_{1} \times \Re_{2}\right)}^{\mathrm{P}}\left(\mathrm{k}, 1_{1}\right) \vee(\mathcal{U})_{\left(\Re_{1} \times \Re_{2}\right)}^{\mathrm{P}}\left(\mathrm{k}, 1_{2}\right) \\
& (\mathcal{F})_{\left(\mathfrak{S}_{1} \times \mathfrak{S}_{2}\right)}^{\mathrm{P}}\left(\left(\mathrm{kl}_{1}\right)\left(\mathrm{kl}_{2}\right)\right)=(\mathcal{F})_{\mathfrak{R}_{1}}^{\mathrm{P}}(\mathrm{k}) \vee(\mathcal{F})_{\mathfrak{S}_{2}}^{\mathrm{P}}\left(\mathrm{l}_{1} \mathrm{l}_{2}\right) \\
& \leq(\mathcal{F})_{\mathfrak{R}_{1}}^{\mathrm{P}}(\mathrm{k}) \vee\left[(\mathcal{F})_{\mathfrak{R}_{2}}^{\mathrm{P}}\left(l_{1}\right) \vee(\mathcal{F})_{\mathfrak{R}_{2}}^{\mathrm{P}}\left(l_{2}\right)\right] \\
& =\left[(\mathcal{F})_{\mathfrak{R}_{1}}^{\mathrm{P}}(\mathrm{k}) \vee(\mathcal{F})_{\mathfrak{R}_{2}}^{\mathrm{P}}\left(\mathrm{l}_{1}\right)\right] \vee\left[(\mathcal{F})_{\mathfrak{R}_{1}}^{\mathrm{P}}(\mathrm{k}) \vee(\mathcal{F})_{\mathfrak{R}_{2}}^{\mathrm{P}}\left(\mathrm{l}_{2}\right)\right] \\
& =(\mathcal{F})_{\left(\Re_{1} \times \Re_{2}\right)}^{\mathrm{P}}\left(\mathrm{k}, 1_{1}\right) \vee(\mathcal{F})_{\left(\Re_{1} \times \Re_{2}\right)}^{\mathrm{P}}\left(\mathrm{k}, 1_{2}\right) \\
& \text { for all }\left(\mathrm{kl}_{1}, \mathrm{kl}_{2}\right) \in \mathcal{G}_{1} \times \mathcal{G}_{2} \text {. }
\end{aligned}
$$

Case 2: for $\mathrm{k} \in \mathfrak{R}_{2}, 1_{1} 1_{2} \in \mathfrak{S}_{1}$.

$$
\begin{aligned}
& (\mathcal{T})_{\left(\mathfrak{S}_{1} \times \mathfrak{S}_{2}\right)}^{\mathrm{P}}\left(\left(\mathrm{l}_{1} \mathrm{k}\right)\left(\mathrm{l}_{2} \mathrm{k}\right)\right)=(\mathcal{T})_{\mathfrak{R}_{2}}^{\mathrm{P}}(\mathrm{k}) \wedge(\mathcal{T})_{\mathfrak{S}_{1}}^{\mathrm{P}}\left(\mathrm{l}_{1} \mathrm{l}_{2}\right) \\
& \leq(\mathcal{T})_{\mathfrak{R}_{2}}^{\mathrm{P}}(\mathrm{k}) \wedge\left[(\mathcal{T})_{\mathfrak{R}_{1}}^{\mathrm{P}}\left(l_{1}\right) \wedge(\mathcal{T})_{\mathfrak{R}_{1}}^{\mathrm{P}}\left(l_{2}\right)\right] \\
& =\left[(\mathcal{T})_{\mathfrak{R}_{2}}^{\mathrm{P}}(\mathrm{k}) \wedge(\mathcal{T})_{\mathfrak{R}_{2}}^{\mathrm{P}}\left(\mathrm{l}_{1}\right)\right] \wedge\left[(\mathcal{T})_{\mathfrak{R}_{2}}^{\mathrm{P}}(\mathrm{k}) \wedge(\mathcal{T})_{\mathfrak{R}_{1}}^{\mathrm{P}}\left(\mathrm{l}_{2}\right)\right] \\
& =(\mathcal{T})_{\left(\Re_{1} \times \Re_{2}\right)}^{\mathrm{P}}\left(\mathrm{l}_{1}, \mathrm{k}\right) \wedge(\mathcal{T})_{\left(\Re_{1} \times \Re_{2}\right)}^{\mathrm{P}}\left(1_{2}, \mathrm{k}\right) \\
& (\mathcal{C})_{\left(\mathfrak{S}_{1} \times \mathfrak{S}_{2}\right)}^{\mathrm{P}}\left(\left(\mathrm{l}_{1} \mathrm{k}\right)\left(\mathrm{l}_{2} \mathrm{k}\right)\right)=(\mathcal{C})_{\mathfrak{R}_{2}}^{\mathrm{P}}(\mathrm{k}) \wedge(\mathcal{C})_{\mathfrak{S}_{1}}^{\mathrm{P}}\left(1_{1} l_{2}\right) \\
& \leq(\mathcal{C})_{\mathfrak{R}_{2}}^{\mathrm{P}}(\mathrm{k}) \wedge\left[(\mathcal{C})_{\mathfrak{R}_{1}}^{\mathrm{P}}\left(\mathrm{l}_{1}\right) \wedge(\mathcal{C})_{\mathfrak{R}_{1}}^{\mathrm{P}}\left(\mathrm{l}_{2}\right)\right] \\
& =\left[(\mathcal{C})_{\mathfrak{R}_{2}}^{\mathrm{P}}(\mathrm{k}) \wedge(\mathcal{C})_{\mathfrak{R}_{2}}^{\mathrm{P}}\left(l_{1}\right)\right] \wedge\left[(\mathcal{C})_{\mathfrak{R}_{2}}^{\mathrm{P}}(\mathrm{k}) \wedge(\mathcal{C})_{\mathfrak{R}_{1}}^{\mathrm{P}}\left(\mathrm{l}_{2}\right)\right] \\
& =(\mathcal{C})_{\left(\Re_{1} \times \Re_{2}\right)}^{\mathrm{P}}\left(\mathrm{l}_{1}, \mathrm{k}\right) \wedge(\mathcal{C})_{\left(\Re_{1} \times \Re_{2}\right)}^{\mathrm{P}}\left(\mathrm{l}_{2}, \mathrm{k}\right) \\
& (\mathcal{U})_{\left(\mathfrak{S}_{1} \times \mathfrak{S}_{2}\right)}^{\mathrm{P}}\left(\left(\mathrm{l}_{1} \mathrm{k}\right)\left(\mathrm{l}_{2} \mathrm{k}\right)\right)=(\mathcal{U})_{\mathfrak{R}_{2}}^{\mathrm{P}}(\mathrm{k}) \vee(\mathcal{U})_{\mathfrak{S}_{1}}^{\mathrm{P}}\left(1_{1} l_{2}\right) \\
& \leq(\mathcal{U})_{\mathfrak{R}_{2}}^{\mathrm{P}}(\mathrm{k}) \vee\left[(\mathcal{U})_{\mathfrak{R}_{1}}^{\mathrm{P}}\left(l_{1}\right) \vee(\mathcal{U})_{\mathfrak{R}_{1}}^{\mathrm{P}}\left(l_{2}\right)\right] \\
& =\left[(\mathcal{U})_{\mathfrak{R}_{2}}^{\mathrm{P}}(\mathrm{k}) \vee(\mathcal{U})_{\mathfrak{R}_{2}}^{\mathrm{P}}\left(1_{1}\right)\right] \vee\left[(\mathcal{U})_{\mathfrak{R}_{2}}^{\mathrm{P}}(\mathrm{k}) \vee(\mathcal{U})_{\mathfrak{R}_{1}}^{\mathrm{P}}\left(l_{2}\right)\right]
\end{aligned}
$$




$$
\begin{aligned}
& =(\mathcal{U})_{\left(\Re_{1} \times \Re_{2}\right)}^{\mathrm{P}}\left(1_{1}, \mathrm{k}\right) \vee(\mathcal{U})_{\left(\Re_{1} \times \Re_{2}\right)}^{\mathrm{P}}\left(\mathrm{l}_{2}, \mathrm{k}\right) \\
& (\mathcal{F})_{\left(\mathfrak{S}_{1} \times \mathfrak{S}_{2}\right)}^{\mathrm{P}}\left(\left(\mathrm{l}_{1} \mathrm{k}\right)\left(\mathrm{l}_{2} \mathrm{k}\right)\right)=(\mathcal{F})_{\mathfrak{R}_{2}}^{\mathrm{P}}(\mathrm{k}) \vee(\mathcal{F})_{\mathfrak{S}_{1}}^{\mathrm{P}}\left(l_{1} l_{2}\right) \\
& \leq(\mathcal{F})_{\mathfrak{R}_{2}}^{\mathrm{P}}(\mathrm{k}) \vee\left[(\mathcal{F})_{\mathfrak{R}_{1}}^{\mathrm{P}}\left(l_{1}\right) \vee(\mathcal{F})_{\mathfrak{R}_{1}}^{\mathrm{P}}\left(l_{2}\right)\right] \\
& =\left[(\mathcal{F})_{\mathfrak{R}_{2}}^{\mathrm{P}}(\mathrm{k}) \vee(\mathcal{F})_{\mathfrak{R}_{2}}^{\mathrm{P}}\left(\mathrm{l}_{1}\right)\right] \vee\left[(\mathcal{F})_{\mathfrak{R}_{2}}^{\mathrm{P}}(\mathrm{k}) \vee(\mathcal{F})_{\mathfrak{R}_{1}}^{\mathrm{P}}\left(\mathrm{l}_{2}\right)\right] \\
& =(\mathcal{F})_{\left(\Re_{1} \times \Re_{2}\right)}^{\mathrm{P}}\left(1_{1}, \mathrm{k}\right) \vee(\mathcal{F})_{\left(\Re_{1} \times \mathfrak{R}_{2}\right)}^{\mathrm{P}}\left(\mathrm{l}_{2}, \mathrm{k}\right),
\end{aligned}
$$

for all $\left(1_{1} \mathrm{k}, 1_{2} \mathrm{k}\right) \in \mathcal{G}_{1} \times \mathcal{G}_{2}$. Likewise, one can prove the negative part.

Definition 3.6 The cross product of two QBSVNGs $\mathcal{G}_{1}$ and $\mathcal{G}_{2}$ is denoted by the pair $\mathcal{G}_{1} \star \mathcal{G}_{2}=$ $\left(\Re_{1} \star \mathfrak{R}_{2}, \mathfrak{S}_{1} \star \mathfrak{S}_{2}\right)$ and defined as:

$$
\begin{aligned}
& \text { (i) }(\mathcal{T})_{\left(\Re_{1} \star \Re_{2}\right)}^{\mathrm{P}}(\mathrm{kl})=(\mathcal{T})_{\mathfrak{R}_{1}}^{\mathrm{P}}(\mathrm{k}) \wedge(\mathcal{T})_{\mathfrak{R}_{2}}^{\mathrm{P}}(\mathrm{l}) \\
& (\mathcal{C})_{\left(\Re_{1} \star \Re_{2}\right)}^{\mathrm{P}}(\mathrm{kl})=(\mathcal{C})_{\mathfrak{R}_{1}}^{\mathrm{P}}(\mathrm{k}) \wedge(\mathcal{C})_{\mathfrak{R}_{2}}^{\mathrm{P}}(\mathrm{l}) \\
& (\mathcal{U})_{\left(\Re_{1} \star \Re_{2}\right)}^{\mathrm{P}}(\mathrm{kl})=(\mathcal{U})_{\mathfrak{R}_{1}}^{\mathrm{P}}(\mathrm{k}) \vee(\mathcal{U})_{\mathfrak{R}_{2}}^{\mathrm{P}}(\mathrm{l}) \\
& (\mathcal{F})_{\left(\Re_{1} \star \Re_{2}\right)}^{\mathrm{P}}(\mathrm{kl})=(\mathcal{F})_{\mathfrak{R}_{1}}^{\mathrm{P}}(\mathrm{k}) \vee(\mathcal{F})_{\mathfrak{R}_{2}}^{\mathrm{P}}(\mathrm{l}) \\
& (\mathcal{T})_{\left(\Re_{1} \star \Re_{2}\right)}^{\mathrm{N}}(\mathrm{kl})=(\mathcal{T})_{\mathfrak{R}_{1}}^{\mathrm{N}}(\mathrm{k}) \vee(\mathcal{T})_{\mathfrak{R}_{2}}^{\mathrm{N}}(\mathrm{l}) \\
& (\mathcal{C})_{\left(\Re_{1} \star \Re_{2}\right)}^{\mathrm{N}}(\mathrm{kl})=(\mathcal{C})_{\mathfrak{R}_{1}}^{\mathrm{N}}(\mathrm{k}) \vee(\mathcal{C})_{\mathfrak{R}_{2}}^{\mathrm{N}}(\mathrm{l}) \\
& (\mathcal{U})_{\left(\Re_{1} \star \Re_{2}\right)}^{\mathrm{N}}(\mathrm{kl})=(\mathcal{U})_{\mathfrak{R}_{1}}^{\mathrm{N}}(\mathrm{k}) \wedge(\mathcal{U})_{\mathfrak{R}_{2}}^{\mathrm{N}}(\mathrm{l}) \\
& (\mathcal{F})_{\left(\Re_{1} \star \Re_{2}\right)}^{\mathrm{N}}(\mathrm{kl})=(\mathcal{F})_{\mathfrak{R}_{1}}^{\mathrm{N}}(\mathrm{k}) \wedge(\mathcal{F})_{\mathfrak{R}_{2}}^{\mathrm{N}}(\mathrm{l})
\end{aligned}
$$

for all $(\mathrm{k}, \mathrm{l}) \in \mathfrak{R}_{1} \star \mathfrak{R}_{2}$.

(ii) $(\mathcal{T})_{\left(\mathfrak{S}_{1} \star \mathfrak{S}_{2}\right)}^{\mathrm{P}}\left(\mathrm{k}_{1} \mathrm{l}_{1}\right)\left(\mathrm{k}_{2} \mathrm{l}_{2}\right)=(\mathcal{T})_{\mathfrak{S}_{1}}^{\mathrm{P}}\left(\mathrm{k}_{1} \mathrm{k}_{2}\right) \wedge(\mathcal{T})_{\mathfrak{S}_{2}}^{\mathrm{P}}\left(\mathrm{l}_{1} \mathrm{l}_{2}\right)$

$(\mathcal{C})_{\left(\mathfrak{S}_{1} \star \mathfrak{S}_{2}\right)}^{\mathrm{P}}\left(\mathrm{k}_{1} \mathrm{l}_{1}\right)\left(\mathrm{k}_{2} \mathrm{l}_{2}\right)=(\mathcal{C})_{\mathfrak{S}_{1}}^{\mathrm{P}}\left(\mathrm{k}_{1} \mathrm{k}_{2}\right) \wedge(\mathcal{C})_{\mathfrak{S}_{2}}^{\mathrm{P}}\left(\mathrm{l}_{1} \mathrm{l}_{2}\right)$

$(\mathcal{U})_{\left(\mathfrak{S}_{1} \star \mathfrak{S}_{2}\right)}^{\mathrm{P}}\left(\mathrm{k}_{1} \mathrm{l}_{1}\right)\left(\mathrm{k}_{2} \mathrm{l}_{2}\right)=(\mathcal{U})_{\mathfrak{S}_{1}}^{\mathrm{P}}\left(\mathrm{k}_{1} \mathrm{k}_{2}\right) \vee(\mathcal{U})_{\mathfrak{S}_{2}}^{\mathrm{P}}\left(\mathrm{l}_{1} \mathrm{l}_{2}\right)$

$(\mathcal{F})_{\left(\mathfrak{S}_{1} \star \mathfrak{S}_{2}\right)}^{\mathrm{P}}\left(\mathrm{k}_{1} \mathrm{l}_{1}\right)\left(\mathrm{k}_{2} \mathrm{l}_{2}\right)=(\mathcal{F})_{\mathfrak{S}_{1}}^{\mathrm{P}}\left(\mathrm{k}_{1} \mathrm{k}_{2}\right) \vee(\mathcal{F})_{\mathfrak{S}_{2}}^{\mathrm{P}}\left(\mathrm{l}_{1} \mathrm{l}_{2}\right)$

$(\mathcal{T})_{\left(\mathfrak{S}_{1} \star \mathfrak{S}_{2}\right)}^{\mathrm{N}}\left(\mathrm{k}_{1} \mathrm{l}_{1}\right)\left(\mathrm{k}_{2} \mathrm{l}_{2}\right)=(\mathcal{T})_{\mathfrak{S}_{1}}^{\mathrm{N}}\left(\mathrm{k}_{1} \mathrm{k}_{2}\right) \vee(\mathcal{T})_{\mathfrak{S}_{2}}^{\mathrm{N}}\left(\mathrm{l}_{1} \mathrm{l}_{2}\right)$

$(\mathcal{C})_{\left(\mathfrak{S}_{1} \star \mathfrak{S}_{2}\right)}^{\mathrm{N}}\left(\mathrm{k}_{1} \mathrm{l}_{1}\right)\left(\mathrm{k}_{2} \mathrm{l}_{2}\right)=(\mathcal{C})_{\mathfrak{S}_{1}}^{\mathrm{N}}\left(\mathrm{k}_{1} \mathrm{k}_{2}\right) \vee(\mathcal{C})_{\mathfrak{S}_{2}}^{\mathrm{N}}\left(\mathrm{l}_{1} \mathrm{l}_{2}\right)$

$(\mathcal{U})_{\left(\mathfrak{S}_{1} \star \mathfrak{S}_{2}\right)}^{\mathrm{N}}\left(\mathrm{k}_{1} \mathrm{l}_{1}\right)\left(\mathrm{k}_{2} \mathrm{l}_{2}\right)=(\mathcal{U})_{\mathfrak{S}_{1}}^{\mathrm{N}}\left(\mathrm{k}_{1} \mathrm{k}_{2}\right) \wedge(\mathcal{U})_{\mathfrak{S}_{2}}^{\mathrm{N}}\left(l_{1} \mathrm{l}_{2}\right)$

$(\mathcal{F})_{\left(\mathfrak{S}_{1} \star \mathfrak{S}_{2}\right)}^{\mathrm{N}}\left(\mathrm{k}_{1} \mathrm{l}_{1}\right)\left(\mathrm{k}_{2} \mathrm{l}_{2}\right)=(\mathcal{F})_{\mathfrak{S}_{1}}^{\mathrm{N}}\left(\mathrm{k}_{1} \mathrm{k}_{2}\right) \wedge(\mathcal{F})_{\mathfrak{S}_{2}}^{\mathrm{N}}\left(\mathrm{l}_{1} \mathrm{l}_{2}\right)$

for all $\mathrm{k}_{1} \mathrm{k}_{2} \in \mathfrak{S}_{1}, 1_{1} \mathrm{l}_{2} \in \mathfrak{S}_{2}$.

Proposition 3.7 The cross product $\mathcal{G}_{1} \star \mathcal{G}_{2}=\left(\mathfrak{R}_{1} \star \mathfrak{R}_{2}, \mathfrak{S}_{1} \star \mathfrak{S}_{2}\right)$ of two QBSVNGs $\mathcal{G}_{1}$ and $\mathcal{G}_{2}$ is also the QBSVNG of $\mathcal{G}_{1} \star \mathcal{G}_{2}$. 
Proof. For all $\left(\mathrm{k}_{1} \mathrm{l}_{1}, \mathrm{k}_{2} \mathrm{l}_{2}\right) \in \mathcal{G}_{1} \star \mathcal{G}_{2}$,

$$
\begin{aligned}
& (\mathcal{T})_{\left(\mathfrak{S}_{1} \star \mathfrak{S}_{2}\right)}^{\mathrm{P}}\left(\left(\mathrm{k}_{1} \mathrm{l}_{1}\right)\left(\mathrm{k}_{2} \mathrm{l}_{2}\right)\right)=(\mathcal{T})_{\mathfrak{S}_{1}}^{\mathrm{P}}\left(\mathrm{k}_{1} \mathrm{k}_{2}\right) \wedge(\mathcal{T})_{\mathfrak{S}_{2}}^{\mathrm{P}}\left(\mathrm{l}_{1} \mathrm{l}_{2}\right) \\
& \leq\left[(\mathcal{T})_{\mathfrak{R}_{1}}^{\mathrm{P}}\left(\mathrm{k}_{1}\right) \wedge(\mathcal{T})_{\mathfrak{R}_{1}}^{\mathrm{P}}\left(\mathrm{k}_{2}\right)\right] \wedge\left[(\mathcal{T})_{\mathfrak{R}_{2}}^{\mathrm{P}}\left(1_{1}\right) \wedge(\mathcal{T})_{\mathfrak{R}_{2}}^{\mathrm{P}}\left(\mathrm{l}_{2}\right)\right] \\
& =\left[(\mathcal{T})_{\mathfrak{R}_{1}}^{\mathrm{P}}\left(\mathrm{k}_{1}\right) \wedge(\mathcal{T})_{\mathfrak{R}_{2}}^{\mathrm{P}}\left(\mathrm{l}_{1}\right)\right] \wedge\left[(\mathcal{T})_{\mathfrak{R}_{1}}^{\mathrm{P}}\left(\mathrm{k}_{2}\right) \wedge(\mathcal{T})_{\mathfrak{R}_{2}}^{\mathrm{P}}\left(\mathrm{l}_{2}\right)\right] \\
& =(\mathcal{T})_{\left(\Re_{1} \star \Re_{2}\right)}^{\mathrm{P}}\left(\mathrm{k}_{1}, 1_{1}\right) \wedge(\mathcal{T})_{\left(\Re_{1} \star \Re_{2}\right)}^{\mathrm{P}}\left(\mathrm{k}_{2}, \mathrm{l}_{2}\right) \\
& (\mathcal{C})_{\left(\mathfrak{S}_{1} \star \mathfrak{S}_{2}\right)}^{\mathrm{P}}\left(\left(\mathrm{k}_{1} \mathrm{l}_{1}\right)\left(\mathrm{k}_{2} \mathrm{l}_{2}\right)\right)=(\mathcal{C})_{\mathfrak{S}_{1}}^{\mathrm{P}}\left(\mathrm{k}_{1} \mathrm{k}_{2}\right) \wedge(\mathcal{C})_{\mathfrak{S}_{2}}^{\mathrm{P}}\left(\mathrm{l}_{1} \mathrm{l}_{2}\right) \\
& \leq\left[(\mathcal{C})_{\mathfrak{R}_{1}}^{\mathrm{P}}\left(\mathrm{k}_{1}\right) \wedge(\mathcal{C})_{\mathfrak{R}_{1}}^{\mathrm{P}}\left(\mathrm{k}_{2}\right)\right] \wedge\left[(\mathcal{C})_{\mathfrak{R}_{2}}^{\mathrm{P}}\left(1_{1}\right) \wedge(\mathcal{C})_{\mathfrak{R}_{2}}^{\mathrm{P}}\left(\mathrm{l}_{2}\right)\right] \\
& =\left[(\mathcal{C})_{\mathfrak{R}_{1}}^{\mathrm{P}}\left(\mathrm{k}_{1}\right) \wedge(\mathcal{C})_{\mathfrak{R}_{2}}^{\mathrm{P}}\left(\mathrm{l}_{1}\right)\right] \wedge\left[(\mathcal{C})_{\mathfrak{R}_{1}}^{\mathrm{P}}\left(\mathrm{k}_{2}\right) \wedge(\mathcal{C})_{\mathfrak{R}_{2}}^{\mathrm{P}}\left(1_{2}\right)\right] \\
& =(\mathcal{C})_{\left(\Re_{1} \star \Re_{2}\right)}^{\mathrm{P}}\left(\mathrm{k}_{1}, 1_{1}\right) \wedge(\mathcal{C})_{\left(\Re_{1} \star \Re_{2}\right)}^{\mathrm{P}}\left(\mathrm{k}_{2}, \mathrm{l}_{2}\right) \\
& (\mathcal{U})_{\left(\mathfrak{S}_{1} \star \mathfrak{S}_{2}\right)}^{\mathrm{P}}\left(\left(\mathrm{k}_{1} \mathrm{l}_{1}\right)\left(\mathrm{k}_{2} \mathrm{l}_{2}\right)\right)=(\mathcal{U})_{\mathfrak{S}_{1}}^{\mathrm{P}}\left(\mathrm{k}_{1} \mathrm{k}_{2}\right) \vee(\mathcal{U})_{\mathfrak{S}_{2}}^{\mathrm{P}}\left(l_{1} l_{2}\right) \\
& \leq\left[(\mathcal{U})_{\mathfrak{R}_{1}}^{\mathrm{P}}\left(\mathrm{k}_{1}\right) \vee(\mathcal{U})_{\mathfrak{R}_{1}}^{\mathrm{P}}\left(\mathrm{k}_{2}\right)\right] \vee\left[(\mathcal{U})_{\mathfrak{R}_{2}}^{\mathrm{P}}\left(\mathrm{l}_{1}\right) \vee(\mathcal{U})_{\mathfrak{R}_{2}}^{\mathrm{P}}\left(\mathrm{l}_{2}\right)\right] \\
& =\left[(\mathcal{U})_{\mathfrak{R}_{1}}^{\mathrm{P}}\left(\mathrm{k}_{1}\right) \vee(\mathcal{U})_{\mathfrak{R}_{2}}^{\mathrm{P}}\left(\mathrm{l}_{1}\right)\right] \vee\left[(\mathcal{U})_{\mathfrak{R}_{1}}^{\mathrm{P}}\left(\mathrm{k}_{2}\right) \vee(\mathcal{U})_{\mathfrak{R}_{2}}^{\mathrm{P}}\left(1_{2}\right)\right] \\
& =(\mathcal{U})_{\left(\Re_{1} \star \Re_{2}\right)}^{\mathrm{P}}\left(\mathrm{k}_{1}, 1_{1}\right) \vee(\mathcal{U})_{\left(\Re_{1} \star \Re_{2}\right)}^{\mathrm{P}}\left(\mathrm{k}_{2}, 1_{2}\right) \\
& (\mathcal{F})_{\left(\mathfrak{S}_{1} \star \mathfrak{S}_{2}\right)}^{\mathrm{P}}\left(\left(\mathrm{k}_{1} \mathrm{l}_{1}\right)\left(\mathrm{k}_{2} \mathrm{l}_{2}\right)\right)=(\mathcal{F})_{\mathfrak{S}_{1}}^{\mathrm{P}}\left(\mathrm{k}_{1} \mathrm{k}_{2}\right) \vee(\mathcal{F})_{\mathfrak{S}_{2}}^{\mathrm{P}}\left(\mathrm{l}_{1} \mathrm{l}_{2}\right) \\
& \leq\left[(\mathcal{F})_{\mathfrak{R}_{1}}^{\mathrm{P}}\left(\mathrm{k}_{1}\right) \vee(\mathcal{F})_{\mathfrak{R}_{1}}^{\mathrm{P}}\left(\mathrm{k}_{2}\right)\right] \vee\left[(\mathcal{F})_{\mathfrak{R}_{2}}^{\mathrm{P}}\left(\mathrm{l}_{1}\right) \vee(\mathcal{F})_{\mathfrak{R}_{2}}^{\mathrm{P}}\left(\mathrm{l}_{2}\right)\right] \\
& =\left[(\mathcal{F})_{\mathfrak{R}_{1}}^{\mathrm{P}}\left(\mathrm{k}_{1}\right) \vee(\mathcal{F})_{\mathfrak{R}_{2}}^{\mathrm{P}}\left(\mathrm{l}_{1}\right)\right] \vee\left[(\mathcal{F})_{\mathfrak{R}_{1}}^{\mathrm{P}}\left(\mathrm{k}_{2}\right) \vee(\mathcal{F})_{\mathfrak{R}_{2}}^{\mathrm{P}}\left(\mathrm{l}_{2}\right)\right] \\
& =(\mathcal{F})_{\left(\Re_{1} \star \Re_{2}\right)}^{\mathrm{P}}\left(\mathrm{k}_{1}, 1_{1}\right) \vee(\mathcal{F})_{\left(\Re_{1} \star \Re_{2}\right)}^{\mathrm{P}}\left(\mathrm{k}_{2}, 1_{2}\right) \text {. }
\end{aligned}
$$

Similarly, one can prove the negative part. This completes the proof.

Definition 3.8 The lexicographic product of two QBVNGs $\mathcal{G}_{1}$ and $\mathcal{G}_{2}$ is denoted by the pair $\mathcal{G}_{1} \cdot \mathcal{G}_{2}=\left(\Re_{1} \cdot \mathfrak{R}_{2}, \mathfrak{S}_{1} \cdot \mathfrak{S}_{2}\right)$ and defined as

$$
\begin{aligned}
& (\mathrm{i})(\mathcal{T})_{\left(\Re_{1} \cdot \Re_{2}\right)}^{\mathrm{P}}(\mathrm{kl})=(\mathcal{T})_{\mathfrak{R}_{1}}^{\mathrm{P}}(\mathrm{k}) \wedge(\mathcal{T})_{\mathfrak{R}_{2}}^{\mathrm{P}}(\mathrm{l}) \\
& (\mathcal{C})_{\left(\Re_{1} \cdot \Re_{2}\right)}^{\mathrm{P}}(\mathrm{kl})=(\mathcal{C})_{\mathfrak{R}_{1}}^{\mathrm{P}}(\mathrm{k}) \wedge(\mathcal{C})_{\mathfrak{R}_{2}}^{\mathrm{P}}(\mathrm{l}) \\
& (\mathcal{U})_{\left(\Re_{1} \cdot \mathfrak{R}_{2}\right)}^{\mathrm{P}}(\mathrm{kl})=(\mathcal{U})_{\mathfrak{R}_{1}}^{\mathrm{P}}(\mathrm{k}) \vee(\mathcal{U})_{\mathfrak{R}_{2}}^{\mathrm{P}}(\mathrm{l}) \\
& (\mathcal{F})_{\left(\Re_{1} \cdot \Re_{2}\right)}^{\mathrm{P}}(\mathrm{kl})=(\mathcal{F})_{\mathfrak{R}_{1}}^{\mathrm{P}}(\mathrm{k}) \vee(\mathcal{F})_{\mathfrak{R}_{2}}^{\mathrm{P}}(\mathrm{l}) \\
& (\mathcal{T})_{\left(\Re_{1} \cdot \mathfrak{R}_{2}\right)}^{\mathrm{N}}(\mathrm{kl})=(\mathcal{T})_{\mathfrak{R}_{1}}^{\mathrm{N}}(\mathrm{k}) \vee(\mathcal{T})_{\mathfrak{R}_{2}}^{\mathrm{N}}(\mathrm{l}) \\
& (\mathcal{C})_{\left(\Re_{1} \cdot \Re_{2}\right)}^{\mathrm{N}}(\mathrm{kl})=(\mathcal{C})_{\mathfrak{R}_{1}}^{\mathrm{N}}(\mathrm{k}) \vee(\mathcal{C})_{\mathfrak{R}_{2}}^{\mathrm{N}}(\mathrm{l}) \\
& (\mathcal{U})_{\left(\Re_{1} \cdot \Re_{2}\right)}^{\mathrm{N}}(\mathrm{kl})=(\mathcal{U})_{\mathfrak{R}_{1}}^{\mathrm{N}}(\mathrm{k}) \wedge(\mathcal{U})_{\mathfrak{R}_{2}}^{\mathrm{N}}(\mathrm{l})
\end{aligned}
$$


$(\mathcal{F})_{\left(\Re_{1} \cdot \Re_{2}\right)}^{\mathrm{N}}(\mathrm{kl})=(\mathcal{F})_{\mathfrak{R}_{1}}^{\mathrm{N}}(\mathrm{k}) \wedge(\mathcal{F})_{\mathfrak{R}_{2}}^{\mathrm{N}}(\mathrm{l})$

for all $(\mathrm{k}, 1) \in \mathfrak{R}_{1} \cdot \mathfrak{R}_{2}$.

(ii) $(\mathcal{T})_{\left(\mathfrak{S}_{1} \cdot \mathfrak{S}_{2}\right)}^{\mathrm{P}}\left(\mathrm{kl}_{1}\right)\left(\mathrm{kl}_{2}\right)=(\mathcal{T})_{\mathfrak{R}_{1}}^{\mathrm{P}}(\mathrm{k}) \wedge(\mathcal{T})_{\mathfrak{S}_{2}}^{\mathrm{P}}\left(\mathrm{l}_{1} \mathrm{l}_{2}\right)$

$(\mathcal{C})_{\left(\mathfrak{S}_{1} \cdot \mathfrak{S}_{2}\right)}^{\mathrm{P}}\left(\mathrm{kl}_{1}\right)\left(\mathrm{kl}_{2}\right)=(\mathcal{C})_{\mathfrak{R}_{1}}^{\mathrm{P}}(\mathrm{k}) \wedge(\mathcal{C})_{\mathfrak{S}_{2}}^{\mathrm{P}}\left(l_{1} \mathrm{l}_{2}\right)$

$(\mathcal{U})_{\left(\mathfrak{S}_{1} \cdot \mathfrak{S}_{2}\right)}^{\mathrm{P}}\left(\mathrm{kl}_{1}\right)\left(\mathrm{kl} \mathrm{l}_{2}\right)=(\mathcal{U})_{\mathfrak{R}_{1}}^{\mathrm{P}}(\mathrm{k}) \vee(\mathcal{U})_{\mathfrak{S}_{2}}^{\mathrm{P}}\left(l_{1} l_{2}\right)$

$(\mathcal{F})_{\left(\mathfrak{S}_{1} \cdot \mathfrak{S}_{2}\right)}^{\mathrm{P}}\left(\mathrm{kl}_{1}\right)\left(\mathrm{kl}_{2}\right)=(\mathcal{F})_{\mathfrak{R}_{1}}^{\mathrm{P}}(\mathrm{k}) \vee(\mathcal{F})_{\mathfrak{S}_{2}}^{\mathrm{P}}\left(\mathrm{l}_{1} \mathrm{l}_{2}\right)$,

$(\mathcal{T})_{\left(\mathfrak{S}_{1} \cdot \mathfrak{S}_{2}\right)}^{\mathrm{N}}\left(\mathrm{kl}_{1}\right)\left(\mathrm{kl}_{2}\right)=(\mathcal{T})_{\mathfrak{R}_{1}}^{\mathrm{N}}(\mathrm{k}) \wedge(\mathcal{T})_{\mathfrak{S}_{2}}^{\mathrm{N}}\left(1_{1} 1_{2}\right)$

$(\mathcal{C})_{\left(\mathfrak{S}_{1} \cdot \mathfrak{S}_{2}\right)}^{\mathrm{N}}\left(\mathrm{kl}_{1}\right)\left(\mathrm{kl}_{2}\right)=(\mathcal{C})_{\mathfrak{R}_{1}}^{\mathrm{N}}(\mathrm{k}) \wedge(\mathcal{C})_{\mathfrak{S}_{2}}^{\mathrm{N}}\left(l_{1} \mathrm{l}_{2}\right)$

$(\mathcal{U})_{\left(\mathfrak{S}_{1} \cdot \mathfrak{S}_{2}\right)}^{\mathrm{N}}\left(\mathrm{kl}_{1}\right)\left(\mathrm{k} l_{2}\right)=(\mathcal{U})_{\mathfrak{R}_{1}}^{\mathrm{N}}(\mathrm{k}) \vee(\mathcal{U})_{\mathfrak{S}_{2}}^{\mathrm{N}}\left(l_{1} l_{2}\right)$

$(\mathcal{F})_{\left(\mathfrak{S}_{1} \cdot \mathfrak{S}_{2}\right)}^{\mathrm{N}}\left(\mathrm{kl}_{1}\right)\left(\mathrm{kl}_{2}\right)=(\mathcal{F})_{\mathfrak{R}_{1}}^{\mathrm{N}}(\mathrm{k}) \vee(\mathcal{F})_{\mathfrak{S}_{2}}^{\mathrm{N}}\left(l_{1} l_{2}\right)$

for all $\mathrm{k} \in \mathfrak{R}_{1}, 1_{1} \mathrm{l}_{2} \in \mathfrak{S}_{2}$.

(iii) $(\mathcal{T})_{\left(\mathfrak{S}_{1} \cdot \mathfrak{S}_{2}\right)}^{\mathrm{P}}\left(\mathrm{k}_{1} \mathrm{l}_{1}\right)\left(\mathrm{k}_{2} \mathrm{l}_{2}\right)=(\mathcal{T})_{\mathfrak{S}_{1}}^{\mathrm{P}}\left(\mathrm{k}_{1} \mathrm{k}_{2}\right) \wedge(\mathcal{T})_{\mathfrak{S}_{2}}^{\mathrm{P}}\left(\mathrm{l}_{1} \mathrm{l}_{2}\right)$

$(\mathcal{C})_{\left(\mathfrak{S}_{1} \cdot \mathfrak{S}_{2}\right)}^{\mathrm{P}}\left(\mathrm{k}_{1} \mathrm{l}_{1}\right)\left(\mathrm{k}_{2} \mathrm{l}_{2}\right)=(\mathcal{C})_{\mathfrak{S}_{1}}^{\mathrm{P}}\left(\mathrm{k}_{1} \mathrm{k}_{2}\right) \wedge(\mathcal{C})_{\mathfrak{S}_{2}}\left(1_{1} \mathrm{l}_{2}\right)$

$(\mathcal{U})_{\left(\mathfrak{S}_{1} \cdot \mathfrak{S}_{2}\right)}^{\mathrm{P}}\left(\mathrm{k}_{1} \mathrm{l}_{1}\right)\left(\mathrm{k}_{2} \mathrm{l}_{2}\right)=(\mathcal{U})_{\mathfrak{S}_{1}}^{\mathrm{P}}\left(\mathrm{k}_{1} \mathrm{k}_{2}\right) \vee(\mathcal{U})_{\mathfrak{S}_{2}}^{\mathrm{P}}\left(\mathrm{l}_{1} \mathrm{l}_{2}\right)$

$(\mathcal{F})_{\left(\mathfrak{S}_{1} \cdot \mathfrak{S}_{2}\right)}^{\mathrm{P}}\left(\mathrm{k}_{1} \mathrm{l}_{1}\right)\left(\mathrm{k}_{2} \mathrm{l}_{2}\right)=(\mathcal{F})_{\mathfrak{S}_{1}}^{\mathrm{P}}\left(\mathrm{k}_{1} \mathrm{k}_{2}\right) \vee(\mathcal{F})_{\mathfrak{S}_{2}}^{\mathrm{P}}\left(l_{1} \mathrm{l}_{2}\right)$,

$(\mathcal{T})_{\left(\mathfrak{S}_{1} \cdot \mathfrak{S}_{2}\right)}^{\mathrm{N}}\left(\mathrm{k}_{1} \mathrm{l}_{1}\right)\left(\mathrm{k}_{2} \mathrm{l}_{2}\right)=(\mathcal{T})_{\mathfrak{S}_{1}}^{\mathrm{N}}\left(\mathrm{k}_{1} \mathrm{k}_{2}\right) \vee(\mathcal{T})_{\mathfrak{S}_{2}}^{\mathrm{N}}\left(\mathrm{l}_{1} \mathrm{l}_{2}\right)$

$(\mathcal{C})_{\left(\mathfrak{S}_{1} \cdot \mathfrak{S}_{2}\right)}^{\mathrm{N}}\left(\mathrm{k}_{1} \mathrm{l}_{1}\right)\left(\mathrm{k}_{2} \mathrm{l}_{2}\right)=(\mathcal{C})_{\mathfrak{S}_{1}}^{\mathrm{N}}\left(\mathrm{k}_{1} \mathrm{k}_{2}\right) \vee(\mathcal{C})_{\mathfrak{S}_{2}}^{\mathrm{N}}\left(l_{1} \mathrm{l}_{2}\right)$

$(\mathcal{U})_{\left(\mathfrak{S}_{1} \cdot \mathfrak{S}_{2}\right)}^{\mathrm{N}}\left(\mathrm{k}_{1} \mathrm{l}_{1}\right)\left(\mathrm{k}_{2} \mathrm{l}_{2}\right)=(\mathcal{U})_{\mathfrak{S}_{1}}^{\mathrm{N}}\left(\mathrm{k}_{1} \mathrm{k}_{2}\right) \wedge(\mathcal{U})_{\mathfrak{S}_{2}}^{\mathrm{N}}\left(\mathrm{l}_{1} \mathrm{l}_{2}\right)$

$(\mathcal{F})_{\left(\mathfrak{S}_{1} \cdot \mathfrak{S}_{2}\right)}^{\mathrm{N}}\left(\mathrm{k}_{1} \mathrm{l}_{1}\right)\left(\mathrm{k}_{2} \mathrm{l}_{2}\right)=(\mathcal{F})_{\mathfrak{S}_{1}}^{\mathrm{N}}\left(\mathrm{k}_{1} \mathrm{k}_{2}\right) \wedge(\mathcal{F})_{\mathfrak{S}_{2}}^{\mathrm{N}}\left(l_{1} \mathrm{l}_{2}\right)$,

for all $\mathrm{k}_{1} \mathrm{k}_{2} \in \mathfrak{S}_{1}, 1_{1} \mathrm{l}_{2} \in \mathfrak{S}_{2}$.

Proposition 3.9 The lexicographic product $\mathcal{G}_{1} \cdot \mathcal{G}_{2}=\left(\mathfrak{R}_{1} \cdot \mathfrak{R}_{2}, \mathfrak{S}_{1} \cdot \mathfrak{S}_{2}\right)$ of two QBSVNGs $\mathcal{G}_{1}$ and $\mathcal{G}_{2}$ is also the QBSVNG of $\mathcal{G}_{1} \cdot \mathcal{G}_{2}$.

Proof. We have two cases.

Case 1: For $\mathrm{k} \in \mathfrak{R}_{1}, \mathrm{l}_{1} \mathrm{l}_{2} \in \mathfrak{S}_{2}$,

$(\mathcal{T})_{\left(\mathfrak{S}_{1} \cdot \mathfrak{S}_{2}\right)}^{\mathrm{P}}\left(\left(\mathrm{kl}_{1}\right)\left(\mathrm{kl}_{2}\right)\right)=(\mathcal{T})_{\mathfrak{R}_{1}}^{\mathrm{P}}(\mathrm{k}) \wedge(\mathcal{T})_{\mathfrak{S}_{2}}^{\mathrm{P}}\left(\mathrm{l}_{1} \mathrm{l}_{2}\right)$

$\leq(\mathcal{T})_{\mathfrak{R}_{1}}^{\mathrm{P}}(\mathrm{k}) \wedge\left[(\mathcal{T})_{\mathfrak{R}_{2}}^{\mathrm{P}}\left(1_{1}\right) \wedge(\mathcal{T})_{\mathfrak{R}_{2}}^{\mathrm{P}}\left(\mathrm{l}_{2}\right)\right]$

$=\left[(\mathcal{T})_{\mathfrak{R}_{1}}^{\mathrm{P}}(\mathrm{k}) \wedge(\mathcal{T})_{\mathfrak{R}_{2}}^{\mathrm{P}}\left(\mathrm{l}_{1}\right)\right] \wedge\left[(\mathcal{T})_{\mathfrak{R}_{1}}^{\mathrm{P}}(\mathrm{k}) \wedge(\mathcal{T})_{\mathfrak{R}_{2}}^{\mathrm{P}}\left(l_{2}\right)\right]$

$=(\mathcal{T})_{\left(\Re_{1} \cdot \Re_{2}\right)}^{\mathrm{P}}\left(\mathrm{k}, 1_{1}\right) \wedge(\mathcal{T})_{\left(\Re_{1} \cdot \Re_{2}\right)}^{\mathrm{P}}\left(\mathrm{k}, 1_{2}\right)$ 


$$
\begin{aligned}
& (\mathcal{C})_{\left(\mathfrak{S}_{1} \cdot \mathfrak{S}_{2}\right)}^{\mathrm{P}}\left(\left(\mathrm{kl}_{1}\right)\left(\mathrm{kl}_{2}\right)\right)=(\mathcal{C})_{\mathfrak{R}_{1}}^{\mathrm{P}}(\mathrm{k}) \wedge(\mathcal{C})_{\mathfrak{S}_{2}}^{\mathrm{P}}\left(\mathrm{l}_{1} \mathrm{l}_{2}\right) \\
& \leq(\mathcal{C})_{\mathfrak{R}_{1}}^{\mathrm{P}}(\mathrm{k}) \wedge\left[(\mathcal{C})_{\mathfrak{R}_{2}}^{\mathrm{P}}\left(\mathrm{l}_{1}\right) \wedge(\mathcal{C})_{\mathfrak{R}_{2}}^{\mathrm{P}}\left(\mathrm{l}_{2}\right)\right] \\
& =\left[(\mathcal{C})_{\mathfrak{R}_{1}}^{\mathrm{P}}(\mathrm{k}) \wedge(\mathcal{C})_{\mathfrak{R}_{2}}^{\mathrm{P}}\left(\mathrm{l}_{1}\right)\right] \wedge\left[(\mathcal{C})_{\mathfrak{R}_{1}}^{\mathrm{P}}(\mathrm{k}) \wedge(\mathcal{C})_{\mathfrak{R}_{2}}^{\mathrm{P}}\left(\mathrm{l}_{2}\right)\right] \\
& =(\mathcal{C})_{\left(\Re_{1} \cdot \Re_{2}\right)}^{\mathrm{P}}\left(\mathrm{k}, 1_{1}\right) \wedge(\mathcal{C})_{\left(\Re_{1} \cdot \Re_{2}\right)}^{\mathrm{P}}\left(\mathrm{k}, 1_{2}\right) \\
& (\mathcal{U})_{\left(\mathfrak{S}_{1} \cdot \mathfrak{S}_{2}\right)}^{\mathrm{P}}\left(\left(\mathrm{kl}_{1}\right)\left(\mathrm{kl}_{2}\right)\right)=(\mathcal{U})_{\mathfrak{R}_{1}}^{\mathrm{P}}(\mathrm{k}) \vee(\mathcal{U})_{\mathfrak{S}_{2}}^{\mathrm{P}}\left(\mathrm{l}_{1} \mathrm{l}_{2}\right) \\
& \leq(\mathcal{U})_{\mathfrak{R}_{1}}^{\mathrm{P}}(\mathrm{k}) \vee\left[(\mathcal{U})_{\mathfrak{R}_{2}}^{\mathrm{P}}\left(l_{1}\right) \vee(\mathcal{U})_{\mathfrak{R}_{2}}^{\mathrm{P}}\left(l_{2}\right)\right] \\
& =\left[(\mathcal{U})_{\mathfrak{R}_{1}}^{\mathrm{P}}(\mathrm{k}) \vee(\mathcal{U})_{\mathfrak{R}_{2}}^{\mathrm{P}}\left(\mathrm{l}_{1}\right)\right] \vee\left[(\mathcal{U})_{\mathfrak{R}_{1}}^{\mathrm{P}}(\mathrm{k}) \vee(\mathcal{U})_{\mathfrak{R}_{2}}^{\mathrm{P}}\left(\mathrm{l}_{2}\right)\right] \\
& =(\mathcal{U})_{\left(\Re_{1} \cdot \Re_{2}\right)}^{\mathrm{P}}\left(\mathrm{k}, 1_{1}\right) \vee(\mathcal{U})_{\left(\Re_{1} \cdot \Re_{2}\right)}^{\mathrm{P}}\left(\mathrm{k}, 1_{2}\right) \\
& (\mathcal{F})_{\left(\mathfrak{S}_{1} \cdot \mathfrak{S}_{2}\right)}^{\mathrm{P}}\left(\left(\mathrm{kl}_{1}\right)\left(\mathrm{kl}_{2}\right)\right)=(\mathcal{F})_{\mathfrak{R}_{1}}^{\mathrm{P}}(\mathrm{k}) \vee(\mathcal{F})_{\mathfrak{S}_{2}}^{\mathrm{P}}\left(\mathrm{l}_{1} \mathrm{l}_{2}\right) \\
& \leq(\mathcal{F})_{\mathfrak{R}_{1}}^{\mathrm{P}}(\mathrm{k}) \vee\left[(\mathcal{F})_{\mathfrak{R}_{2}}^{\mathrm{P}}\left(\mathrm{l}_{1}\right) \vee(\mathcal{F})_{\mathfrak{R}_{2}}^{\mathrm{P}}\left(l_{2}\right)\right] \\
& =\left[(\mathcal{F})_{\mathfrak{R}_{1}}^{\mathrm{P}}(\mathrm{k}) \vee(\mathcal{F})_{\mathfrak{R}_{2}}^{\mathrm{P}}\left(\mathrm{l}_{1}\right)\right] \vee\left[(\mathcal{F})_{\mathfrak{R}_{1}}^{\mathrm{P}}(\mathrm{k}) \vee(\mathcal{F})_{\mathfrak{R}_{2}}^{\mathrm{P}}\left(\mathrm{l}_{2}\right)\right] \\
& =(\mathcal{F})_{\left(\Re_{1} \cdot \Re_{2}\right)}^{\mathrm{P}}\left(\mathrm{k}, 1_{1}\right) \vee(\mathcal{F})_{\left(\Re_{1} \cdot \Re_{2}\right)}^{\mathrm{P}}\left(\mathrm{k}, \mathrm{l}_{2}\right) \text {, }
\end{aligned}
$$

for all $\left(\mathrm{kl}_{1}, \mathrm{kl}_{2}\right) \in \mathfrak{S}_{1} \cdot \mathfrak{S}_{2}$.

Case 2: For all $\mathrm{k}_{1} \mathrm{l}_{1} \in \mathfrak{S}_{1}, \mathrm{k}_{2} \mathrm{l}_{2} \in \mathfrak{S}_{2}$,

$$
\begin{aligned}
& (\mathcal{T})_{\left(\mathfrak{S}_{1} \cdot \mathfrak{S}_{2}\right)}^{\mathrm{P}}\left(\left(\mathrm{k}_{1} \mathrm{l}_{1}\right)\left(\mathrm{k}_{2} \mathrm{l}_{2}\right)\right)=(\mathcal{T})_{\mathfrak{S}_{1}}^{\mathrm{P}}\left(\mathrm{k}_{1} \mathrm{k}_{2}\right) \wedge(\mathcal{T})_{\mathfrak{S}_{2}}^{\mathrm{P}}\left(\mathrm{l}_{1} \mathrm{l}_{2}\right) \\
& \leq\left[(\mathcal{T})_{\mathfrak{R}_{1}}^{\mathrm{P}}\left(\mathrm{k}_{1}\right) \wedge(\mathcal{T})_{\mathfrak{R}_{1}}^{\mathrm{P}}\left(\mathrm{k}_{2}\right)\right] \wedge\left[(\mathcal{T})_{\mathfrak{R}_{2}}^{\mathrm{P}}\left(\mathrm{l}_{1}\right) \wedge(\mathcal{T})_{\mathfrak{R}_{2}}^{\mathrm{P}}\left(\mathrm{l}_{2}\right)\right] \\
& =\left[(\mathcal{T})_{\mathfrak{R}_{1}}^{\mathrm{P}}\left(\mathrm{k}_{1}\right) \wedge(\mathcal{T})_{\mathfrak{R}_{2}}^{\mathrm{P}}\left(\mathrm{l}_{1}\right)\right] \wedge\left[(\mathcal{T})_{\mathfrak{R}_{1}}^{\mathrm{P}}\left(\mathrm{k}_{2}\right) \wedge(\mathcal{T})_{\mathfrak{R}_{2}}^{\mathrm{P}}\left(\mathrm{l}_{2}\right)\right] \\
& =(\mathcal{T})_{\left(\Re_{1} \cdot \Re_{2}\right)}^{\mathrm{P}}\left(\mathrm{k}_{1}, 1_{1}\right) \wedge(\mathcal{T})_{\left(\Re_{1} \cdot \Re_{2}\right)}^{\mathrm{P}}\left(\mathrm{k}_{2}, 1_{2}\right) \\
& (\mathcal{C})_{\left(\mathfrak{S}_{1} \cdot \mathfrak{S}_{2}\right)}^{\mathrm{P}}\left(\left(\mathrm{k}_{1} \mathrm{l}_{1}\right)\left(\mathrm{k}_{2} \mathrm{l}_{2}\right)\right)=(\mathcal{C})_{\mathfrak{S}_{1}}^{\mathrm{P}}\left(\mathrm{k}_{1} \mathrm{k}_{2}\right) \wedge(\mathcal{C})_{\mathfrak{S}_{2}}^{\mathrm{P}}\left(\mathrm{l}_{1} \mathrm{l}_{2}\right) \\
& \leq\left[(\mathcal{C})_{\mathfrak{R}_{1}}^{\mathrm{P}}\left(\mathrm{k}_{1}\right) \wedge(\mathcal{C})_{\mathfrak{R}_{1}}^{\mathrm{P}}\left(\mathrm{k}_{2}\right)\right] \wedge\left[(\mathcal{C})_{\mathfrak{R}_{2}}^{\mathrm{P}}\left(\mathrm{l}_{1}\right) \wedge(\mathcal{C})_{\mathfrak{R}_{2}}^{\mathrm{P}}\left(\mathrm{l}_{2}\right)\right] \\
& =\left[(\mathcal{C})_{\mathfrak{R}_{1}}^{\mathrm{P}}\left(\mathrm{k}_{1}\right) \wedge(\mathcal{C})_{\mathfrak{R}_{2}}^{\mathrm{P}}\left(\mathrm{l}_{1}\right)\right] \wedge\left[(\mathcal{C})_{\mathfrak{R}_{1}}^{\mathrm{P}}\left(\mathrm{k}_{2}\right) \wedge(\mathcal{C})_{\mathfrak{R}_{2}}^{\mathrm{P}}\left(l_{2}\right)\right] \\
& =(\mathcal{C})_{\left(\Re_{1} \cdot \Re_{2}\right)}^{\mathrm{P}}\left(\mathrm{k}_{1}, 1_{1}\right) \wedge(\mathcal{C})_{\left(\Re_{1} \cdot \Re_{2}\right)}^{\mathrm{P}}\left(\mathrm{k}_{2}, \mathrm{l}_{2}\right) \\
& (\mathcal{U})_{\left(\mathfrak{S}_{1} \cdot \mathfrak{S}_{2}\right)}^{\mathrm{P}}\left(\left(\mathrm{k}_{1} \mathrm{l}_{1}\right)\left(\mathrm{k}_{2} \mathrm{l}_{2}\right)\right)=(\mathcal{U})_{\mathfrak{S}_{1}}^{\mathrm{P}}\left(\mathrm{k}_{1} \mathrm{k}_{2}\right) \vee(\mathcal{U})_{\mathfrak{S}_{2}}^{\mathrm{P}}\left(l_{1} \mathrm{l}_{2}\right) \\
& \leq\left[(\mathcal{U})_{\mathfrak{R}_{1}}^{\mathrm{P}}\left(\mathrm{k}_{1}\right) \vee(\mathcal{U})_{\mathfrak{R}_{1}}^{\mathrm{P}}\left(\mathrm{k}_{2}\right)\right] \vee\left[(\mathcal{U})_{\mathfrak{R}_{2}}^{\mathrm{P}}\left(l_{1}\right) \vee(\mathcal{U})_{\mathfrak{R}_{2}}^{\mathrm{P}}\left(\mathrm{l}_{2}\right)\right] \\
& =\left[(\mathcal{U})_{\mathfrak{R}_{1}}^{\mathrm{P}}\left(\mathrm{k}_{1}\right) \vee(\mathcal{U})_{\mathfrak{R}_{2}}^{\mathrm{P}}\left(\mathrm{l}_{1}\right)\right] \vee\left[(\mathcal{U})_{\mathfrak{R}_{1}}^{\mathrm{P}}\left(\mathrm{k}_{2}\right) \vee(\mathcal{U})_{\mathfrak{R}_{2}}^{\mathrm{P}}\left(1_{2}\right)\right] \\
& =(\mathcal{U})_{\left(\Re_{1} \cdot \Re_{2}\right)}^{\mathrm{P}}\left(\mathrm{k}_{1}, 1_{1}\right) \vee(\mathcal{U})_{\left(\Re_{1} \cdot \Re_{2}\right)}^{\mathrm{P}}\left(\mathrm{k}_{2}, 1_{2}\right) \\
& (\mathcal{F})_{\left(\mathfrak{S}_{1} \cdot \mathfrak{S}_{2}\right)}^{\mathrm{P}}\left(\left(\mathrm{k}_{1} \mathrm{l}_{1}\right)\left(\mathrm{k}_{2} \mathrm{l}_{2}\right)\right)=(\mathcal{F})_{\mathfrak{S}_{1}}^{\mathrm{P}}\left(\mathrm{k}_{1} \mathrm{k}_{2}\right) \vee(\mathcal{F})_{\mathfrak{S}_{2}}^{\mathrm{P}}\left(l_{1} \mathrm{l}_{2}\right)
\end{aligned}
$$




$$
\begin{aligned}
& \leq\left[(\mathcal{F})_{\mathfrak{R}_{1}}^{\mathrm{P}}\left(\mathrm{k}_{1}\right) \vee(\mathcal{F})_{\mathfrak{R}_{1}}^{\mathrm{P}}\left(\mathrm{k}_{2}\right)\right] \vee\left[(\mathcal{F})_{\mathfrak{R}_{2}}^{\mathrm{P}}\left(\mathrm{l}_{1}\right) \vee(\mathcal{F})_{\mathfrak{R}_{2}}^{\mathrm{P}}\left(\mathrm{l}_{2}\right)\right] \\
& =\left[(\mathcal{F})_{\mathfrak{R}_{1}}^{\mathrm{P}}\left(\mathrm{k}_{1}\right) \vee(\mathcal{F})_{\mathfrak{R}_{2}}^{\mathrm{P}}\left(\mathrm{l}_{1}\right)\right] \vee\left[(\mathcal{F})_{\mathfrak{R}_{1}}^{\mathrm{P}}\left(\mathrm{k}_{2}\right) \vee(\mathcal{F})_{\mathfrak{R}_{2}}^{\mathrm{P}}\left(\mathrm{l}_{2}\right)\right] \\
& =(\mathcal{F})_{\left(\mathfrak{R}_{1} \cdot \mathfrak{R}_{2}\right)}^{\mathrm{P}}\left(\mathrm{k}_{1}, 1_{1}\right) \vee(\mathcal{F})_{\left(\mathfrak{R}_{1} \cdot \mathfrak{R}_{2}\right)}^{\mathrm{P}}\left(\mathrm{k}_{2}, \mathrm{l}_{2}\right),
\end{aligned}
$$

for all $\left(\mathrm{k}_{1} \mathrm{l}_{1}, \mathrm{k}_{2} \mathrm{l}_{2}\right) \in \mathfrak{R}_{1} \cdot \mathfrak{R}_{2}$. Likewise, one can prove the negative part. This completes the proof.

Definition 3.10 The strong product of two QBSVNGs $\mathcal{G}_{1}$ and $\mathcal{G}_{2}$ is denoted by the pair $\mathcal{G}_{1} \otimes$ $\mathcal{G}_{2}=\left(\mathfrak{R}_{1} \otimes \mathfrak{R}_{2}, \mathfrak{S}_{1} \otimes \mathfrak{S}_{2}\right)$ and defined as

$$
\begin{aligned}
& \text { (i) }(\mathcal{T})_{\left(\Re_{1} \otimes \mathfrak{R}_{2}\right)}^{\mathrm{P}}(\mathrm{kl})=(\mathcal{T})_{\mathfrak{R}_{1}}^{\mathrm{P}}(\mathrm{k}) \wedge(\mathcal{T})_{\mathfrak{R}_{2}}^{\mathrm{P}}(\mathrm{l}) \\
& (\mathcal{C})_{\left(\Re_{1} \otimes \mathfrak{R}_{2}\right)}^{\mathrm{P}}(\mathrm{kl})=(\mathcal{C})_{\mathfrak{R}_{1}}^{\mathrm{P}}(\mathrm{k}) \wedge(\mathcal{C})_{\mathfrak{R}_{2}}^{\mathrm{P}}(\mathrm{l}) \\
& (\mathcal{U})_{\left(\Re_{1} \otimes \Re_{2}\right)}^{\mathrm{P}}(\mathrm{kl})=(\mathcal{U})_{\mathfrak{R}_{1}}^{\mathrm{P}}(\mathrm{k}) \vee(\mathcal{U})_{\mathfrak{R}_{2}}^{\mathrm{P}}(\mathrm{l}) \\
& (\mathcal{F})_{\left(\Re_{1} \bowtie \Re_{2}\right)}^{\mathrm{P}}(\mathrm{kl})=(\mathcal{F})_{\mathfrak{R}_{1}}^{\mathrm{P}}(\mathrm{k}) \vee(\mathcal{F})_{\mathfrak{R}_{2}}^{\mathrm{P}}(\mathrm{l}) \\
& (\mathcal{T})_{\left(\Re_{1} \otimes \Re_{2}\right)}^{\mathrm{N}}(\mathrm{kl})=(\mathcal{T})_{\mathfrak{R}_{1}}^{\mathrm{N}}(\mathrm{k}) \vee(\mathcal{T})_{\mathfrak{R}_{2}}^{\mathrm{N}}(\mathrm{l}) \\
& (\mathcal{C})_{\left(\Re_{1} \otimes \mathfrak{R}_{2}\right)}^{\mathrm{N}}(\mathrm{kl})=(\mathcal{C})_{\mathfrak{R}_{1}}^{\mathrm{N}}(\mathrm{k}) \vee(\mathcal{C})_{\mathfrak{R}_{2}}^{\mathrm{N}}(\mathrm{l}) \\
& (\mathcal{U})_{\left(\Re_{1} \otimes \Re_{2}\right)}^{\mathrm{N}}(\mathrm{kl})=(\mathcal{U})_{\mathfrak{R}_{1}}^{\mathrm{N}}(\mathrm{k}) \wedge(\mathcal{U})_{\mathfrak{R}_{2}}^{\mathrm{N}}(\mathrm{l}) \\
& (\mathcal{F})_{\left(\Re_{1} \otimes \Re_{2}\right)}^{\mathrm{N}}(\mathrm{kl})=(\mathcal{F})_{\mathfrak{R}_{1}}^{\mathrm{N}}(\mathrm{k}) \wedge(\mathcal{F})_{\mathfrak{R}_{2}}^{\mathrm{N}}(\mathrm{l}),
\end{aligned}
$$

for all $(\mathrm{k}, \mathrm{l}) \in \mathfrak{R}_{1} \otimes \mathfrak{R}_{2}$.

(ii) $(\mathcal{T})_{\left(\mathfrak{S}_{1} \otimes \mathfrak{S}_{2}\right)}^{\mathrm{P}}\left(\mathrm{kl}_{1}\right)\left(\mathrm{kl}_{2}\right)=(\mathcal{T})_{\mathfrak{R}_{1}}^{\mathrm{P}}(\mathrm{k}) \wedge(\mathcal{T})_{\mathfrak{S}_{2}}^{\mathrm{P}}\left(\mathrm{l}_{1} \mathrm{l}_{2}\right)$

$(\mathcal{C})_{\left(\mathfrak{S}_{1} \otimes \mathfrak{S}_{2}\right)}^{\mathrm{P}}\left(\mathrm{kl}_{1}\right)\left(\mathrm{kl}_{2}\right)=(\mathcal{C})_{\mathfrak{R}_{1}}^{\mathrm{P}}(\mathrm{k}) \wedge(\mathcal{C})_{\mathfrak{S}_{2}}^{\mathrm{P}}\left(\mathrm{l}_{1} \mathrm{l}_{2}\right)$

$(\mathcal{U})_{\left(\mathfrak{S}_{1} \otimes \mathfrak{S}_{2}\right)}^{\mathrm{P}}\left(\mathrm{kl}_{1}\right)\left(\mathrm{kl}_{2}\right)=(\mathcal{U})_{\mathfrak{R}_{1}}^{\mathrm{P}}(\mathrm{k}) \vee(\mathcal{U})_{\mathfrak{S}_{2}}^{\mathrm{P}}\left(1_{1} 1_{2}\right)$

$(\mathcal{F})_{\left(\mathfrak{S}_{1} \otimes \mathfrak{S}_{2}\right)}^{\mathrm{P}}\left(\mathrm{kl}_{1}\right)\left(\mathrm{kl}_{2}\right)=(\mathcal{F})_{\mathfrak{R}_{1}}^{\mathrm{P}}(\mathrm{k}) \vee(\mathcal{F})_{\mathfrak{S}_{2}}^{\mathrm{P}}\left(\mathrm{l}_{1} \mathrm{l}_{2}\right)$

$(\mathcal{T})_{\left(\mathfrak{S}_{1} \otimes \mathfrak{S}_{2}\right)}^{\mathrm{N}}\left(\mathrm{kl}_{1}\right)\left(\mathrm{kl}_{2}\right)=(\mathcal{T})_{\mathfrak{R}_{1}}^{\mathrm{N}}(\mathrm{k}) \vee(\mathcal{T})_{\mathfrak{S}_{2}}^{\mathrm{N}}\left(\mathrm{l}_{1} \mathrm{l}_{2}\right)$

$(\mathcal{C})_{\left(\mathfrak{S}_{1} \otimes \mathfrak{S}_{2}\right)}^{\mathrm{N}}\left(\mathrm{kl}_{1}\right)\left(\mathrm{kl}_{2}\right)=(\mathcal{C})_{\mathfrak{R}_{1}}^{\mathrm{N}}(\mathrm{k}) \vee(\mathcal{C})_{\mathfrak{S}_{2}}^{\mathrm{N}}\left(\mathrm{l}_{1} \mathrm{l}_{2}\right)$

$(\mathcal{U})_{\left(\mathfrak{S}_{1} \otimes \mathfrak{S}_{2}\right)}^{\mathrm{N}}\left(\mathrm{kl}_{1}\right)\left(\mathrm{kl}_{2}\right)=(\mathcal{U})_{\mathfrak{R}_{1}}^{\mathrm{N}}(\mathrm{k}) \wedge(\mathcal{U})_{\mathfrak{S}_{2}}^{\mathrm{P}}\left(1_{1} \mathrm{l}_{2}\right)$

$(\mathcal{F})_{\left(\mathfrak{S}_{1} \otimes \mathfrak{S}_{2}\right)}^{\mathrm{N}}\left(\mathrm{kl}_{1}\right)\left(\mathrm{kl}_{2}\right)=(\mathcal{F})_{\mathfrak{R}_{1}}^{\mathrm{N}}(\mathrm{k}) \wedge(\mathcal{F})_{\mathfrak{S}_{2}}^{\mathrm{N}}\left(l_{1} \mathrm{l}_{2}\right)$,

for all $\mathrm{k} \in \mathfrak{R}_{1}, \mathrm{l}_{1} \mathrm{l}_{2} \in \mathfrak{S}_{2}$.

(iii) $(\mathcal{T})_{\left(\mathfrak{S}_{1} \otimes \mathfrak{S}_{2}\right)}^{\mathrm{P}}\left(\mathrm{k}_{1} \mathrm{l}\right)\left(\mathrm{k}_{2} \mathrm{l}\right)=(\mathcal{T})_{\mathfrak{R}_{2}}^{\mathrm{P}}(\mathrm{l}) \wedge(\mathcal{T})_{\mathfrak{S}_{1}}^{\mathrm{P}}\left(\mathrm{k}_{1} \mathrm{k}_{2}\right)$

$(\mathcal{C})_{\left(\mathfrak{S}_{1} \otimes \mathfrak{S}_{2}\right)}^{\mathrm{P}}\left(\mathrm{k}_{1} \mathrm{l}\right)\left(\mathrm{k}_{2} \mathrm{l}\right)=(\mathcal{C})_{\mathfrak{R}_{2}}^{\mathrm{P}}(\mathrm{l}) \wedge(\mathcal{C})_{\mathfrak{S}_{1}}^{\mathrm{P}}\left(\mathrm{k}_{1} \mathrm{k}_{2}\right)$

$(\mathcal{U})_{\left(\mathfrak{S}_{1} \otimes \mathfrak{S}_{2}\right)}^{\mathrm{P}}\left(\mathrm{k}_{1} \mathrm{l}\right)\left(\mathrm{k}_{2} \mathrm{l}\right)=(\mathcal{U})_{\mathfrak{R}_{2}}^{\mathrm{P}}(\mathrm{l}) \vee(\mathcal{U})_{\mathfrak{S}_{1}}^{\mathrm{P}}\left(\mathrm{k}_{1} \mathrm{k}_{2}\right)$

$(\mathcal{F})_{\left(\mathfrak{S}_{1} \otimes \mathfrak{S}_{2}\right)}^{\mathrm{P}}\left(\mathrm{k}_{1} \mathrm{l}\right)\left(\mathrm{k}_{2} \mathrm{l}\right)=(\mathcal{F})_{\mathfrak{R}_{2}}^{\mathrm{P}}(\mathrm{l}) \vee(\mathcal{F})_{\mathfrak{S}_{1}}^{\mathrm{P}}\left(\mathrm{k}_{1} \mathrm{k}_{2}\right)$ 
$(\mathcal{T})_{\left(\mathfrak{S}_{1} \otimes \mathfrak{S}_{2}\right)}^{\mathrm{N}}\left(\mathrm{k}_{1} \mathrm{l}\right)\left(\mathrm{k}_{2} \mathrm{l}\right)=(\mathcal{T})_{\mathfrak{R}_{2}}^{\mathrm{N}}(\mathrm{l}) \vee(\mathcal{T})_{\mathfrak{S}_{1}}^{\mathrm{N}}\left(\mathrm{k}_{1} \mathrm{k}_{2}\right)$

$(\mathcal{C})_{\left(\mathfrak{S}_{1} \otimes \mathfrak{S}_{2}\right)}^{\mathrm{N}}\left(\mathrm{k}_{1} \mathrm{l}\right)\left(\mathrm{k}_{2} \mathrm{l}\right)=(\mathcal{C})_{\mathfrak{R}_{2}}^{\mathrm{N}}(\mathrm{l}) \vee(\mathcal{C})_{\mathfrak{S}_{1}}^{\mathrm{N}}\left(\mathrm{k}_{1} \mathrm{k}_{2}\right)$

$(\mathcal{U})_{\left(\mathfrak{S}_{1} \otimes \mathfrak{S}_{2}\right)}^{\mathrm{N}}\left(\mathrm{k}_{1} \mathrm{l}\right)\left(\mathrm{k}_{2} \mathrm{l}\right)=(\mathcal{U})_{\mathfrak{R}_{2}}^{\mathrm{N}}(\mathrm{l}) \wedge(\mathcal{U})_{\mathfrak{S}_{1}}^{\mathrm{N}}\left(\mathrm{k}_{1} \mathrm{k}_{2}\right)$

$(\mathcal{F})_{\left(\mathfrak{S}_{1} \bowtie \mathfrak{S}_{2}\right)}^{\mathrm{N}}\left(\mathrm{k}_{1} \mathrm{l}\right)\left(\mathrm{k}_{2} \mathrm{l}\right)=(\mathcal{F})_{\mathfrak{R}_{2}}^{\mathrm{N}}(\mathrm{l}) \wedge(\mathcal{F})_{\mathfrak{S}_{1}}^{\mathrm{N}}\left(\mathrm{k}_{1} \mathrm{k}_{2}\right)$,

for all $1 \in \mathfrak{R}_{2}, \mathrm{k}_{1} \mathrm{k}_{2} \in \mathfrak{S}_{1}$.

$(\mathrm{iv})(\mathcal{T})_{\left(\mathfrak{S}_{1} \otimes \mathfrak{S}_{2}\right)}^{\mathrm{P}}\left(\mathrm{k}_{1} \mathrm{l}_{1}\right)\left(\mathrm{k}_{2} \mathrm{l}_{2}\right)=(\mathcal{T})_{\mathfrak{S}_{1}}^{\mathrm{P}}\left(\mathrm{k}_{1} \mathrm{k}_{2}\right) \wedge(\mathcal{T})_{\mathfrak{S}_{2}}^{\mathrm{P}}\left(\mathrm{l}_{1} \mathrm{l}_{2}\right)$

$(\mathcal{C})_{\left(\mathfrak{S}_{1} \otimes \mathfrak{S}_{2}\right)}^{\mathrm{P}}\left(\mathrm{k}_{1} \mathrm{l}_{1}\right)\left(\mathrm{k}_{2} \mathrm{l}_{2}\right)=(\mathcal{C})_{\mathfrak{S}_{1}}^{\mathrm{P}}\left(\mathrm{k}_{1} \mathrm{k}_{2}\right) \wedge(\mathcal{C})_{\mathfrak{S}_{2}}^{\mathrm{P}}\left(\mathrm{l}_{1} \mathrm{l}_{2}\right)$

$(\mathcal{U})_{\left(\mathfrak{S}_{1} \otimes \mathfrak{S}_{2}\right)}^{\mathrm{P}}\left(\mathrm{k}_{1} \mathrm{l}_{1}\right)\left(\mathrm{k}_{2} \mathrm{l}_{2}\right)=(\mathcal{U})_{\mathfrak{S}_{1}}^{\mathrm{P}}\left(\mathrm{k}_{1} \mathrm{k}_{2}\right) \vee(\mathcal{U})_{\mathfrak{S}_{2}}^{\mathrm{P}}\left(\mathrm{l}_{1} \mathrm{l}_{2}\right)$

$(\mathcal{F})_{\left(\mathfrak{S}_{1} \otimes \mathfrak{S}_{2}\right)}^{\mathrm{P}}\left(\mathrm{k}_{1} \mathrm{l}_{1}\right)\left(\mathrm{k}_{2} \mathrm{l}_{2}\right)=(\mathcal{F})_{\mathfrak{S}_{1}}^{\mathrm{P}}\left(\mathrm{k}_{1} \mathrm{k}_{2}\right) \vee(\mathcal{F})_{\mathfrak{S}_{2}}^{\mathrm{P}}\left(\mathrm{l}_{1} \mathrm{l}_{2}\right)$

$(\mathcal{T})_{\left(\mathfrak{S}_{1} \otimes \mathfrak{S}_{2}\right)}^{\mathrm{N}}\left(\mathrm{k}_{1} \mathrm{l}_{1}\right)\left(\mathrm{k}_{2} \mathrm{l}_{2}\right)=(\mathcal{T})_{\mathfrak{S}_{1}}^{\mathrm{N}}\left(\mathrm{k}_{1} \mathrm{k}_{2}\right) \vee(\mathcal{T})_{\mathfrak{S}_{2}}^{\mathrm{N}}\left(\mathrm{l}_{1} \mathrm{l}_{2}\right)$

$(\mathcal{C})_{\left(\mathfrak{S}_{1} \otimes \mathfrak{S}_{2}\right)}^{\mathrm{N}}\left(\mathrm{k}_{1} \mathrm{l}_{1}\right)\left(\mathrm{k}_{2} \mathrm{l}_{2}\right)=(\mathcal{C})_{\mathfrak{S}_{1}}^{\mathrm{N}}\left(\mathrm{k}_{1} \mathrm{k}_{2}\right) \vee(\mathcal{C})_{\mathfrak{S}_{2}}^{\mathrm{N}}\left(\mathrm{l}_{1} \mathrm{l}_{2}\right)$

$(\mathcal{U})_{\left(\mathfrak{S}_{1} \otimes \mathfrak{S}_{2}\right)}^{\mathrm{N}}\left(\mathrm{k}_{1} \mathrm{l}_{1}\right)\left(\mathrm{k}_{2} \mathrm{l}_{2}\right)=(\mathcal{U})_{\mathfrak{S}_{1}}^{\mathrm{N}}\left(\mathrm{k}_{1} \mathrm{k}_{2}\right) \wedge(\mathcal{U})_{\mathfrak{S}_{2}}^{\mathrm{N}}\left(\mathrm{l}_{1} \mathrm{l}_{2}\right)$

$(\mathcal{F})_{\left(\mathfrak{S}_{1} \otimes \mathfrak{S}_{2}\right)}^{\mathrm{N}}\left(\mathrm{k}_{1} \mathrm{l}_{1}\right)\left(\mathrm{k}_{2} \mathrm{l}_{2}\right)=(\mathcal{F})_{\mathfrak{S}_{1}}^{\mathrm{N}}\left(\mathrm{k}_{1} \mathrm{k}_{2}\right) \wedge(\mathcal{F})_{\mathfrak{S}_{2}}^{\mathrm{N}}\left(1_{1} \mathrm{l}_{2}\right)$

for all $\mathrm{k}_{1} \mathrm{k}_{2} \in \mathfrak{S}_{1}, \mathrm{l}_{1} \mathrm{l}_{2} \in \mathfrak{S}_{2}$.

Proposition 3.11 The strong product $\mathcal{G}_{1} \otimes \mathcal{G}_{2}=\left(\mathfrak{R}_{1} \otimes \mathfrak{R}_{2}, \mathfrak{S}_{1} \otimes \mathfrak{S}_{2}\right)$ of two QBSVNG $\mathcal{G}_{1}$ and $\mathcal{G}_{2}$ is also the QBSVNG of $\mathcal{G}_{1} \otimes \mathcal{G}_{2}$.

Proof. We have three cases.

Case 1: For $\mathrm{k} \in \mathfrak{R}_{1}, \mathrm{l}_{1} \mathrm{l}_{2} \in \mathfrak{S}_{2}$,

$(\mathcal{T})_{\left(\mathfrak{S}_{1} \otimes \mathfrak{S}_{2}\right)}^{\mathrm{P}}\left(\left(\mathrm{kl}_{1}\right)\left(\mathrm{kl}_{2}\right)\right)=(\mathcal{T})_{\mathfrak{R}_{1}}^{\mathrm{P}}(\mathrm{k}) \wedge(\mathcal{T})_{\mathfrak{S}_{2}}^{\mathrm{P}}\left(\mathrm{l}_{1} \mathrm{l}_{2}\right)$

$\leq(\mathcal{T})_{\mathfrak{R}_{1}}^{\mathrm{P}}(\mathrm{k}) \wedge\left[(\mathcal{T})_{\mathfrak{R}_{2}}^{\mathrm{P}}\left(\mathrm{l}_{1}\right) \wedge(\mathcal{T})_{\mathfrak{R}_{2}}^{\mathrm{P}}\left(1_{2}\right)\right]$

$=\left[(\mathcal{T})_{\mathfrak{R}_{1}}^{\mathrm{P}}(\mathrm{k}) \wedge(\mathcal{T})_{\mathfrak{R}_{2}}^{\mathrm{P}}\left(\mathrm{l}_{1}\right)\right] \wedge\left[(\mathcal{T})_{\mathfrak{R}_{1}}^{\mathrm{P}}(\mathrm{k}) \wedge(\mathcal{T})_{\mathfrak{R}_{2}}^{\mathrm{P}}\left(\mathrm{l}_{2}\right)\right]$

$=(\mathcal{T})_{\left(\Re_{1} \bowtie \Re_{2}\right)}^{\mathrm{P}}\left(\mathrm{k}, 1_{1}\right) \wedge(\mathcal{T})_{\left(\Re_{1} \bowtie \Re_{2}\right)}^{\mathrm{P}}\left(\mathrm{k}, 1_{2}\right)$

$(\mathcal{C})_{\left(\mathfrak{S}_{1} \otimes \mathfrak{S}_{2}\right)}^{\mathrm{P}}\left(\left(\mathrm{kl}_{1}\right)\left(\mathrm{kl}_{2}\right)\right)=(\mathcal{C})_{\mathfrak{R}_{1}}^{\mathrm{P}}(\mathrm{k}) \wedge(\mathcal{C})_{\mathfrak{S}_{2}}^{\mathrm{P}}\left(\mathrm{l}_{1} \mathrm{l}_{2}\right)$

$\leq(\mathcal{C})_{\mathfrak{R}_{1}}^{\mathrm{P}}(\mathrm{k}) \wedge\left[(\mathcal{C})_{\mathfrak{R}_{2}}^{\mathrm{P}}\left(\mathrm{l}_{1}\right) \wedge(\mathcal{C})_{\mathfrak{R}_{2}}^{\mathrm{P}}\left(\mathrm{l}_{2}\right)\right]$

$=\left[(\mathcal{C})_{\mathfrak{R}_{1}}^{\mathrm{P}}(\mathrm{k}) \wedge(\mathcal{C})_{\mathfrak{R}_{2}}^{\mathrm{P}}\left(\mathrm{l}_{1}\right)\right] \wedge\left[(\mathcal{C})_{\mathfrak{R}_{1}}^{\mathrm{P}}(\mathrm{k}) \wedge(\mathcal{C})_{\mathfrak{R}_{2}}^{\mathrm{P}}\left(\mathrm{l}_{2}\right)\right]$

$=(\mathcal{C})_{\left(\Re_{1} \otimes \Re_{2}\right)}^{\mathrm{P}}\left(\mathrm{k}, 1_{1}\right) \wedge(\mathcal{C})_{\left(\Re_{1} \otimes \Re_{2}\right)}^{\mathrm{P}}\left(\mathrm{k}, 1_{2}\right)$

$(\mathcal{U})_{\left(\mathfrak{S}_{1} \bowtie \mathfrak{S}_{2}\right)}^{\mathrm{P}}\left(\left(\mathrm{kl}_{1}\right)\left(\mathrm{kl}_{2}\right)\right)=(\mathcal{U})_{\mathfrak{R}_{1}}^{\mathrm{P}}(\mathrm{k}) \vee(\mathcal{U})_{\mathfrak{S}_{2}}^{\mathrm{P}}\left(l_{1} \mathrm{l}_{2}\right)$

$\leq(\mathcal{U})_{\mathfrak{R}_{1}}^{\mathrm{P}}(\mathrm{k}) \vee\left[(\mathcal{U})_{\mathfrak{R}_{2}}^{\mathrm{P}}\left(1_{1}\right) \vee(\mathcal{U})_{\mathfrak{R}_{2}}^{\mathrm{P}}\left(1_{2}\right)\right]$ 


$$
\begin{aligned}
& =\left[(\mathcal{U})_{\mathfrak{R}_{1}}^{\mathrm{P}}(\mathrm{k}) \vee(\mathcal{U})_{\mathfrak{R}_{2}}^{\mathrm{P}}\left(1_{1}\right)\right] \vee\left[(\mathcal{U})_{\mathfrak{R}_{1}}^{\mathrm{P}}(\mathrm{k}) \vee(\mathcal{U})_{\mathfrak{R}_{2}}^{\mathrm{P}}\left(l_{2}\right)\right] \\
& =(\mathcal{U})_{\left(\Re_{1} \otimes \Re_{2}\right)}^{\mathrm{P}}\left(\mathrm{k}, 1_{1}\right) \vee(\mathcal{U})_{\left(\Re_{1} \otimes \Re_{2}\right)}^{\mathrm{P}}\left(\mathrm{k}, 1_{2}\right) \\
& (\mathcal{F})_{\left(\mathfrak{S}_{1} \otimes \mathfrak{S}_{2}\right)}^{\mathrm{P}}\left(\left(\mathrm{kl}_{1}\right)\left(\mathrm{kl}_{2}\right)\right)=(\mathcal{F})_{\mathfrak{R}_{1}}^{\mathrm{P}}(\mathrm{k}) \vee(\mathcal{F})_{\mathfrak{S}_{2}}^{\mathrm{P}}\left(\mathrm{l}_{1} \mathrm{l}_{2}\right) \\
& \leq(\mathcal{F})_{\mathfrak{R}_{1}}^{\mathrm{P}}(\mathrm{k}) \vee\left[(\mathcal{F})_{\mathfrak{R}_{2}}^{\mathrm{P}}\left(l_{1}\right) \vee(\mathcal{F})_{\mathfrak{R}_{2}}^{\mathrm{P}}\left(l_{2}\right)\right] \\
& =\left[(\mathcal{F})_{\mathfrak{R}_{1}}^{\mathrm{P}}(\mathrm{k}) \vee(\mathcal{F})_{\mathfrak{R}_{2}}^{\mathrm{P}}\left(\mathrm{l}_{1}\right)\right] \vee\left[(\mathcal{F})_{\mathfrak{R}_{1}}^{\mathrm{P}}(\mathrm{k}) \vee(\mathcal{F})_{\mathfrak{R}_{2}}^{\mathrm{P}}\left(1_{2}\right)\right] \\
& =(\mathcal{F})_{\left(\Re_{1} \otimes \Re_{2}\right)}^{\mathrm{P}}\left(\mathrm{k}, 1_{1}\right) \vee(\mathcal{F})_{\left(\Re_{1} \otimes \Re_{2}\right)}^{\mathrm{P}}\left(\mathrm{k}, 1_{2}\right),
\end{aligned}
$$

for all $\left(\mathrm{kl}_{1}, \mathrm{kl}_{2}\right) \in \mathfrak{R}_{1} \otimes \mathfrak{R}_{2}$.

Case 2: For $\mathrm{k} \in \mathfrak{R}_{2}, 1_{1} \mathrm{l}_{2} \in \mathfrak{S}_{1}$.

$$
\begin{aligned}
& (\mathcal{T})_{\left(\mathfrak{S}_{1} \otimes \mathfrak{S}_{2}\right)}^{\mathrm{P}}\left(\left(\mathrm{l}_{1} \mathrm{k}\right)\left(\mathrm{l}_{2} \mathrm{k}\right)\right)=(\mathcal{T})_{\mathfrak{R}_{2}}^{\mathrm{P}}(\mathrm{k}) \wedge(\mathcal{T})_{\mathfrak{S}_{1}}^{\mathrm{P}}\left(1_{1} l_{2}\right) \\
& \leq(\mathcal{T})_{\mathfrak{R}_{2}}^{\mathrm{P}}(\mathrm{k}) \wedge\left[(\mathcal{T})_{\mathfrak{R}_{1}}^{\mathrm{P}}\left(l_{1}\right) \wedge(\mathcal{T})_{\mathfrak{R}_{1}}^{\mathrm{P}}\left(l_{2}\right)\right] \\
& =\left[(\mathcal{T})_{\mathfrak{R}_{2}}^{\mathrm{P}}(\mathrm{k}) \wedge(\mathcal{T})_{\mathfrak{R}_{2}}^{\mathrm{P}}\left(\mathrm{l}_{1}\right)\right] \wedge\left[(\mathcal{T})_{\mathfrak{R}_{2}}^{\mathrm{P}}(\mathrm{k}) \wedge(\mathcal{T})_{\mathfrak{R}_{1}}^{\mathrm{P}}\left(l_{2}\right)\right] \\
& =(\mathcal{T})_{\left(\Re_{1} \otimes \Re_{2}\right)}^{\mathrm{P}}\left(l_{1}, \mathrm{k}\right) \wedge(\mathcal{T})_{\left(\Re_{1} \otimes \mathfrak{R}_{2}\right)}^{\mathrm{P}}\left(\mathrm{l}_{2}, \mathrm{k}\right) \\
& (\mathcal{C})_{\left(\mathfrak{S}_{1} \otimes \mathfrak{S}_{2}\right)}^{\mathrm{P}}\left(\left(\mathrm{l}_{1} \mathrm{k}\right)\left(\mathrm{l}_{2} \mathrm{k}\right)\right)=(\mathcal{C})_{\mathfrak{R}_{2}}^{\mathrm{P}}(\mathrm{k}) \wedge(\mathcal{C})_{\mathfrak{S}_{1}}^{\mathrm{P}}\left(1_{1} \mathrm{l}_{2}\right) \\
& \leq(\mathcal{C})_{\mathfrak{R}_{2}}^{\mathrm{P}}(\mathrm{k}) \wedge\left[(\mathcal{C})_{\mathfrak{R}_{1}}^{\mathrm{P}}\left(\mathrm{l}_{1}\right) \wedge(\mathcal{C})_{\mathfrak{R}_{1}}^{\mathrm{P}}\left(\mathrm{l}_{2}\right)\right] \\
& =\left[(\mathcal{C})_{\mathfrak{R}_{2}}^{\mathrm{P}}(\mathrm{k}) \wedge(\mathcal{C})_{\mathfrak{R}_{2}}^{\mathrm{P}}\left(\mathrm{l}_{1}\right)\right] \wedge\left[(\mathcal{C})_{\mathfrak{R}_{2}}^{\mathrm{P}}(\mathrm{k}) \wedge(\mathcal{C})_{\mathfrak{R}_{1}}^{\mathrm{P}}\left(\mathrm{l}_{2}\right)\right] \\
& =(\mathcal{C})_{\left(\Re_{1} \otimes \Re_{2}\right)}^{\mathrm{P}}\left(l_{1}, \mathrm{k}\right) \wedge(\mathcal{C})_{\left(\Re_{1} \otimes \Re_{2}\right)}^{\mathrm{P}}\left(\mathrm{l}_{2}, \mathrm{k}\right) \\
& (\mathcal{U})_{\left(\mathfrak{S}_{1} \otimes \mathfrak{S}_{2}\right)}^{\mathrm{P}}\left(\left(1_{1} \mathrm{k}\right)\left(\mathrm{l}_{2} \mathrm{k}\right)\right)=(\mathcal{U})_{\mathfrak{R}_{2}}^{\mathrm{P}}(\mathrm{k}) \vee(\mathcal{U})_{\mathfrak{S}_{1}}^{\mathrm{P}}\left(1_{1} l_{2}\right) \\
& \leq(\mathcal{U})_{\mathfrak{R}_{2}}^{\mathrm{P}}(\mathrm{k}) \vee\left[(\mathcal{U})_{\mathfrak{R}_{1}}^{\mathrm{P}}\left(1_{1}\right) \vee(\mathcal{U})_{\mathfrak{R}_{1}}^{\mathrm{P}}\left(l_{2}\right)\right] \\
& =\left[(\mathcal{U})_{\mathfrak{R}_{2}}^{\mathrm{P}}(\mathrm{k}) \vee(\mathcal{U})_{\mathfrak{R}_{2}}^{\mathrm{P}}\left(1_{1}\right)\right] \vee\left[(\mathcal{U})_{\mathfrak{R}_{2}}^{\mathrm{P}}(\mathrm{k}) \vee(\mathcal{U})_{\mathfrak{R}_{1}}^{\mathrm{P}}\left(1_{2}\right)\right] \\
& =(\mathcal{U})_{\left(\Re_{1} \varangle \Re_{2}\right)}^{\mathrm{P}}\left(1_{1}, \mathrm{k}\right) \vee(\mathcal{U})_{\left(\Re_{1} \otimes \Re_{2}\right)}^{\mathrm{P}}\left(\mathrm{l}_{2}, \mathrm{k}\right) \\
& (\mathcal{F})_{\left(\mathfrak{S}_{1} \otimes \mathfrak{S}_{2}\right)}^{\mathrm{P}}\left(\left(\mathrm{l}_{1} \mathrm{k}\right)\left(\mathrm{l}_{2} \mathrm{k}\right)\right)=(\mathcal{F})_{\mathfrak{R}_{2}}^{\mathrm{P}}(\mathrm{k}) \vee(\mathcal{F})_{\mathfrak{S}_{1}}^{\mathrm{P}}\left(l_{1} l_{2}\right) \\
& \leq(\mathcal{F})_{\mathfrak{R}_{2}}^{\mathrm{P}}(\mathrm{k}) \vee\left[(\mathcal{F})_{\mathfrak{R}_{1}}^{\mathrm{P}}\left(l_{1}\right) \vee(\mathcal{F})_{\mathfrak{R}_{1}}^{\mathrm{P}}\left(l_{2}\right)\right] \\
& =\left[(\mathcal{F})_{\mathfrak{R}_{2}}^{\mathrm{P}}(\mathrm{k}) \vee(\mathcal{F})_{\mathfrak{R}_{2}}^{\mathrm{P}}\left(\mathrm{l}_{1}\right)\right] \vee\left[(\mathcal{F})_{\mathfrak{R}_{2}}^{\mathrm{P}}(\mathrm{k}) \vee(\mathcal{F})_{\mathfrak{R}_{1}}^{\mathrm{P}}\left(\mathrm{l}_{2}\right)\right] \\
& =(\mathcal{F})_{\left(\Re_{1} \otimes \mathfrak{R}_{2}\right)}^{\mathrm{P}}\left(\mathrm{l}_{1}, \mathrm{k}\right) \vee(\mathcal{F})_{\left(\Re_{1} \otimes \mathfrak{R}_{2}\right)}^{\mathrm{P}}\left(l_{2}, \mathrm{k}\right),
\end{aligned}
$$

for all $\left(1_{1} \mathrm{k}, 1_{2} \mathrm{k}\right) \in \mathcal{G}_{1} \otimes \mathcal{G}_{2}$. 
Case 3: For all $\mathrm{k}_{1} \mathrm{l}_{1} \in \mathfrak{S}_{1}, \mathrm{k}_{2} \mathrm{l}_{2} \in \mathfrak{S}_{2}$,

$$
\begin{aligned}
& (\mathcal{T})_{\left(\mathfrak{S}_{1} \otimes \mathfrak{S}_{2}\right)}^{\mathrm{P}}\left(\left(\mathrm{k}_{1} \mathrm{l}_{1}\right)\left(\mathrm{k}_{2} \mathrm{l}_{2}\right)\right)=(\mathcal{T})_{\mathfrak{S}_{1}}^{\mathrm{P}}\left(\mathrm{k}_{1} \mathrm{k}_{2}\right) \wedge(\mathcal{T})_{\mathfrak{S}_{2}}^{\mathrm{P}}\left(\mathrm{l}_{1} \mathrm{l}_{2}\right) \\
& \leq\left[(\mathcal{T})_{\mathfrak{R}_{1}}^{\mathrm{P}}\left(\mathrm{k}_{1}\right) \wedge(\mathcal{T})_{\mathfrak{R}_{1}}^{\mathrm{P}}\left(\mathrm{k}_{2}\right)\right] \wedge\left[(\mathcal{T})_{\mathfrak{R}_{2}}^{\mathrm{P}}\left(1_{1}\right) \wedge(\mathcal{T})_{\mathfrak{R}_{2}}^{\mathrm{P}}\left(\mathrm{l}_{2}\right)\right] \\
& =\left[(\mathcal{T})_{\mathfrak{R}_{1}}^{\mathrm{P}}\left(\mathrm{k}_{1}\right) \wedge(\mathcal{T})_{\mathfrak{R}_{2}}^{\mathrm{P}}\left(\mathrm{l}_{1}\right)\right] \wedge\left[(\mathcal{T})_{\mathfrak{R}_{1}}^{\mathrm{P}}\left(\mathrm{k}_{2}\right) \wedge(\mathcal{T})_{\mathfrak{R}_{2}}^{\mathrm{P}}\left(\mathrm{l}_{2}\right)\right] \\
& =(\mathcal{T})_{\left(\Re_{1} \otimes \Re_{2}\right)}^{\mathrm{P}}\left(\mathrm{k}_{1}, 1_{1}\right) \wedge(\mathcal{T})_{\left(\Re_{1} \otimes \mathfrak{R}_{2}\right)}^{\mathrm{P}}\left(\mathrm{k}_{2}, \mathrm{l}_{2}\right) \\
& (\mathcal{C})_{\left(\mathfrak{S}_{1} \otimes \mathfrak{S}_{2}\right)}^{\mathrm{P}}\left(\left(\mathrm{k}_{1} \mathrm{l}_{1}\right)\left(\mathrm{k}_{2} \mathrm{l}_{2}\right)\right)=(\mathcal{C})_{\mathfrak{S}_{1}}^{\mathrm{P}}\left(\mathrm{k}_{1} \mathrm{k}_{2}\right) \wedge(\mathcal{C})_{\mathfrak{S}_{2}}^{\mathrm{P}}\left(\mathrm{l}_{1} \mathrm{l}_{2}\right) \\
& \leq\left[(\mathcal{C})_{\mathfrak{R}_{1}}^{\mathrm{P}}\left(\mathrm{k}_{1}\right) \wedge(\mathcal{C})_{\mathfrak{R}_{1}}^{\mathrm{P}}\left(\mathrm{k}_{2}\right)\right] \wedge\left[(\mathcal{C})_{\mathfrak{R}_{2}}^{\mathrm{P}}\left(\mathrm{l}_{1}\right) \wedge(\mathcal{C})_{\mathfrak{R}_{2}}^{\mathrm{P}}\left(\mathrm{l}_{2}\right)\right] \\
& =\left[(\mathcal{C})_{\mathfrak{R}_{1}}^{\mathrm{P}}\left(\mathrm{k}_{1}\right) \wedge(\mathcal{C})_{\mathfrak{R}_{2}}^{\mathrm{P}}\left(\mathrm{l}_{1}\right)\right] \wedge\left[(\mathcal{C})_{\mathfrak{R}_{1}}^{\mathrm{P}}\left(\mathrm{k}_{2}\right) \wedge(\mathcal{C})_{\mathfrak{R}_{2}}^{\mathrm{P}}\left(1_{2}\right)\right] \\
& =(\mathcal{C})_{\left(\Re_{1} \otimes \Re_{2}\right)}^{\mathrm{P}}\left(\mathrm{k}_{1}, 1_{1}\right) \wedge(\mathcal{C})_{\left(\Re_{1} \otimes \mathfrak{R}_{2}\right)}^{\mathrm{P}}\left(\mathrm{k}_{2}, 1_{2}\right) \\
& (\mathcal{U})_{\left(\mathfrak{S}_{1} \otimes \mathfrak{S}_{2}\right)}^{\mathrm{P}}\left(\left(\mathrm{k}_{1} \mathrm{l}_{1}\right)\left(\mathrm{k}_{2} \mathrm{l}_{2}\right)\right)=(\mathcal{U})_{\mathfrak{S}_{1}}^{\mathrm{P}}\left(\mathrm{k}_{1} \mathrm{k}_{2}\right) \vee(\mathcal{U})_{\mathfrak{S}_{2}}^{\mathrm{P}}\left(\mathrm{l}_{1} \mathrm{l}_{2}\right) \\
& \leq\left[(\mathcal{U})_{\mathfrak{R}_{1}}^{\mathrm{P}}\left(\mathrm{k}_{1}\right) \vee(\mathcal{U})_{\mathfrak{R}_{1}}^{\mathrm{P}}\left(\mathrm{k}_{2}\right)\right] \vee\left[(\mathcal{U})_{\mathfrak{R}_{2}}^{\mathrm{P}}\left(\mathrm{l}_{1}\right) \vee(\mathcal{U})_{\mathfrak{R}_{2}}^{\mathrm{P}}\left(\mathrm{l}_{2}\right)\right] \\
& =\left[(\mathcal{U})_{\mathfrak{R}_{1}}^{\mathrm{P}}\left(\mathrm{k}_{1}\right) \vee(\mathcal{U})_{\mathfrak{R}_{2}}^{\mathrm{P}}\left(\mathrm{l}_{1}\right)\right] \vee\left[(\mathcal{U})_{\mathfrak{R}_{1}}^{\mathrm{P}}\left(\mathrm{k}_{2}\right) \vee(\mathcal{U})_{\mathfrak{R}_{2}}^{\mathrm{P}}\left(1_{2}\right)\right] \\
& =(\mathcal{U})_{\left(\Re_{1} \otimes \Re_{2}\right)}^{\mathrm{P}}\left(\mathrm{k}_{1}, 1_{1}\right) \vee(\mathcal{U})_{\left(\Re_{1} \otimes \Re_{2}\right)}^{\mathrm{P}}\left(\mathrm{k}_{2}, 1_{2}\right) \\
& (\mathcal{F})_{\left(\mathfrak{S}_{1} \otimes \mathfrak{S}_{2}\right)}^{\mathrm{P}}\left(\left(\mathrm{k}_{1} \mathrm{l}_{1}\right)\left(\mathrm{k}_{2} \mathrm{l}_{2}\right)\right)=(\mathcal{F})_{\mathfrak{S}_{1}}^{\mathrm{P}}\left(\mathrm{k}_{1} \mathrm{k}_{2}\right) \vee(\mathcal{F})_{\mathfrak{S}_{2}}^{\mathrm{P}}\left(\mathrm{l}_{1} \mathrm{l}_{2}\right) \\
& \leq\left[(\mathcal{F})_{\mathfrak{R}_{1}}^{\mathrm{P}}\left(\mathrm{k}_{1}\right) \vee(\mathcal{F})_{\mathfrak{R}_{1}}^{\mathrm{P}}\left(\mathrm{k}_{2}\right)\right] \vee\left[(\mathcal{F})_{\mathfrak{R}_{2}}^{\mathrm{P}}\left(\mathrm{l}_{1}\right) \vee(\mathcal{F})_{\mathfrak{R}_{2}}^{\mathrm{P}}\left(\mathrm{l}_{2}\right)\right] \\
& =\left[(\mathcal{F})_{\mathfrak{R}_{1}}^{\mathrm{P}}\left(\mathrm{k}_{1}\right) \vee(\mathcal{F})_{\mathfrak{R}_{2}}^{\mathrm{P}}\left(\mathrm{l}_{1}\right)\right] \vee\left[(\mathcal{F})_{\mathfrak{R}_{1}}^{\mathrm{P}}\left(\mathrm{k}_{2}\right) \vee(\mathcal{F})_{\mathfrak{R}_{2}}^{\mathrm{P}}\left(\mathrm{l}_{2}\right)\right] \\
& =(\mathcal{F})_{\left(\Re_{1} \otimes \Re_{2}\right)}^{\mathrm{P}}\left(\mathrm{k}_{1}, 1_{1}\right) \vee(\mathcal{F})_{\left(\Re_{1} \otimes \Re_{2}\right)}^{\mathrm{P}}\left(\mathrm{k}_{2}, 1_{2}\right) \text {, }
\end{aligned}
$$

for all $\left(\mathrm{k}_{1} \mathrm{l}_{1}, \mathrm{k}_{2} \mathrm{l}_{2}\right) \in \mathfrak{R}_{1} \otimes \mathfrak{R}_{2}$. Likewise, one can prove the negative part. This completes the proof.

Definition 3.12 The composition of two QBSVNGs $\mathcal{G}_{1}$ and $\mathcal{G}_{2}$ is denoted by the pair $\mathcal{G}_{1} \circ \mathcal{G}_{2}=$ $\left(\mathfrak{R}_{1} \circ \mathfrak{R}_{2}, \mathfrak{S}_{1} \circ \mathfrak{S}_{2}\right)$ and defined as

(i) $(\mathcal{T})_{\left(\Re_{1} \circ \Re_{2}\right)}^{\mathrm{P}}(\mathrm{kl})=(\mathcal{T})_{\mathfrak{R}_{1}}^{\mathrm{P}}(\mathrm{k}) \wedge(\mathcal{T})_{\mathfrak{R}_{2}}^{\mathrm{P}}(\mathrm{l})$

$(\mathcal{C})_{\left(\Re_{1} \circ \Re_{2}\right)}^{\mathrm{P}}(\mathrm{kl})=(\mathcal{C})_{\mathfrak{R}_{1}}^{\mathrm{P}}(\mathrm{k}) \wedge(\mathcal{C})_{\mathfrak{R}_{2}}^{\mathrm{P}}(\mathrm{l})$

$(\mathcal{U})_{\left(\Re_{1} \circ \Re_{2}\right)}^{\mathrm{P}}(\mathrm{kl})=(\mathcal{U})_{\mathfrak{R}_{1}}^{\mathrm{P}}(\mathrm{k}) \vee(\mathcal{U})_{\mathfrak{R}_{2}}^{\mathrm{P}}(\mathrm{l})$

$(\mathcal{F})_{\left(\Re_{1} \circ \Re_{2}\right)}^{\mathrm{P}}(\mathrm{kl})=(\mathcal{F})_{\mathfrak{R}_{1}}^{\mathrm{P}}(\mathrm{k}) \vee(\mathcal{F})_{\mathfrak{R}_{2}}^{\mathrm{P}}(\mathrm{l})$

$(\mathcal{T})_{\left(\Re_{1} \circ \Re_{2}\right)}^{\mathrm{N}}(\mathrm{kl})=(\mathcal{T})_{\mathfrak{R}_{1}}^{\mathrm{N}}(\mathrm{k}) \vee(\mathcal{T})_{\mathfrak{R}_{2}}^{\mathrm{N}}(\mathrm{l})$

$(\mathcal{C})_{\left(\Re_{1} \circ \Re_{2}\right)}^{\mathrm{N}}(\mathrm{kl})=(\mathcal{C})_{\Re_{1}}^{\mathrm{N}}(\mathrm{k}) \vee(\mathcal{C})_{\mathfrak{R}_{2}}^{\mathrm{N}}(\mathrm{l})$

$(\mathcal{U})_{\left(\Re_{1} \circ \Re_{2}\right)}^{\mathrm{N}}(\mathrm{kl})=(\mathcal{U})_{\mathfrak{R}_{1}}^{\mathrm{N}}(\mathrm{k}) \wedge(\mathcal{U})_{\mathfrak{R}_{2}}^{\mathrm{N}}(\mathrm{l})$

$(\mathcal{F})_{\left(\Re_{1} \circ \Re_{2}\right)}^{\mathrm{N}}(\mathrm{kl})=(\mathcal{F})_{\mathfrak{R}_{1}}^{\mathrm{N}}(\mathrm{k}) \wedge(\mathcal{F})_{\mathfrak{R}_{2}}^{\mathrm{N}}(\mathrm{l})$, 
for all $(\mathrm{k}, 1) \in \mathfrak{R}_{1} \circ \mathfrak{R}_{2}$.

(ii) $(\mathcal{T})_{\left(\mathfrak{S}_{1} \circ \mathfrak{S}_{2}\right)}^{\mathrm{P}}\left(\mathrm{kl}_{1}\right)\left(\mathrm{kl}_{2}\right)=(\mathcal{T})_{\mathfrak{R}_{1}}^{\mathrm{P}}(\mathrm{k}) \wedge(\mathcal{T})_{\mathfrak{S}_{2}}^{\mathrm{P}}\left(l_{1} \mathrm{l}_{2}\right)$

$(\mathcal{C})_{\left(\mathfrak{S}_{1} \circ \mathfrak{S}_{2}\right)}^{\mathrm{P}}\left(\mathrm{kl}_{1}\right)\left(\mathrm{kl}_{2}\right)=(\mathcal{C})_{\mathfrak{R}_{1}}^{\mathrm{P}}(\mathrm{k}) \wedge(\mathcal{C})_{\mathfrak{S}_{2}}^{\mathrm{P}}\left(\mathrm{l}_{1} \mathrm{l}_{2}\right)$

$(\mathcal{U})_{\left(\mathfrak{S}_{1} \circ \mathfrak{S}_{2}\right)}^{\mathrm{P}}\left(\mathrm{kl}_{1}\right)\left(\mathrm{kl}_{2}\right)=(\mathcal{U})_{\mathfrak{R}_{1}}^{\mathrm{P}}(\mathrm{k}) \vee(\mathcal{U})_{\mathfrak{S}_{2}}^{\mathrm{P}}\left(\mathrm{l}_{1} \mathrm{l}_{2}\right)$

$(\mathcal{F})_{\left(\mathfrak{S}_{1} \circ \mathfrak{S}_{2}\right)}^{\mathrm{P}}\left(\mathrm{kl}_{1}\right)\left(\mathrm{kl}_{2}\right)=(\mathcal{F})_{\mathfrak{R}_{1}}^{\mathrm{P}}(\mathrm{k}) \vee(\mathcal{F})_{\mathfrak{S}_{2}}^{\mathrm{P}}\left(\mathrm{l}_{1} \mathrm{l}_{2}\right)$

$(\mathcal{T})_{\left(\mathfrak{S}_{1} \circ \mathfrak{S}_{2}\right)}^{\mathrm{N}}\left(\mathrm{kl}_{1}\right)\left(\mathrm{kl}_{2}\right)=(\mathcal{T})_{\mathfrak{R}_{1}}^{\mathrm{N}}(\mathrm{k}) \vee(\mathcal{T})_{\mathfrak{S}_{2}}^{\mathrm{N}}\left(l_{1} l_{2}\right)$

$(\mathcal{C})_{\left(\mathfrak{S}_{1} \circ \mathfrak{S}_{2}\right)}^{\mathrm{N}}\left(\mathrm{kl}_{1}\right)\left(\mathrm{kl}_{2}\right)=(\mathcal{C})_{\mathfrak{R}_{1}}^{\mathrm{N}}(\mathrm{k}) \vee(\mathcal{C})_{\mathfrak{S}_{2}}^{\mathrm{N}}\left(l_{1} l_{2}\right)$

$(\mathcal{U})_{\left(\mathfrak{S}_{1} \circ \mathfrak{S}_{2}\right)}^{\mathrm{N}}\left(\mathrm{kl}_{1}\right)\left(\mathrm{kl}_{2}\right)=(\mathcal{U})_{\mathfrak{R}_{1}}^{\mathrm{N}}(\mathrm{k}) \wedge(\mathcal{U})_{\mathfrak{S}_{2}}^{\mathrm{N}}\left(l_{1} \mathrm{l}_{2}\right)$

$(\mathcal{F})_{\left(\mathfrak{S}_{1} \circ \mathfrak{S}_{2}\right)}^{\mathrm{N}}\left(\mathrm{kl}_{1}\right)\left(\mathrm{kl}_{2}\right)=(\mathcal{F})_{\mathfrak{R}_{1}}^{\mathrm{N}}(\mathrm{k}) \wedge(\mathcal{F})_{\mathfrak{S}_{2}}^{\mathrm{N}}\left(1_{1} 1_{2}\right)$,

for all $\mathrm{k} \in \mathfrak{R}_{1}, 1_{1} 1_{2} \in \mathfrak{S}_{2}$.

(iii) $(\mathcal{T})_{\left(\mathfrak{S}_{1} \circ \mathfrak{S}_{2}\right)}^{\mathrm{P}}\left(\mathrm{k}_{1} \mathrm{l}\right)\left(\mathrm{k}_{2} \mathrm{l}\right)=(\mathcal{T})_{\mathfrak{R}_{2}}^{\mathrm{P}}(\mathrm{l}) \wedge(\mathcal{T})_{\mathfrak{S}_{1}}^{\mathrm{P}}\left(\mathrm{k}_{1} \mathrm{k}_{2}\right)$

$(\mathcal{C})_{\left(\mathfrak{S}_{1} \circ \mathfrak{S}_{2}\right)}^{\mathrm{P}}\left(\mathrm{k}_{1} \mathrm{l}\right)\left(\mathrm{k}_{2} \mathrm{l}\right)=(\mathcal{C})_{\mathfrak{R}_{2}}^{\mathrm{P}}(\mathrm{l}) \wedge(\mathcal{C})_{\mathfrak{S}_{1}}^{\mathrm{P}}\left(\mathrm{k}_{1} \mathrm{k}_{2}\right)$

$(\mathcal{U})_{\left(\mathfrak{S}_{1} \circ \mathfrak{S}_{2}\right)}^{\mathrm{P}}\left(\mathrm{k}_{1} \mathrm{l}\right)\left(\mathrm{k}_{2} \mathrm{l}\right)=(\mathcal{U})_{\mathfrak{R}_{2}}^{\mathrm{P}}(\mathrm{l}) \vee(\mathcal{U})_{\mathfrak{S}_{1}}^{\mathrm{P}}\left(\mathrm{k}_{1} \mathrm{k}_{2}\right)$

$(\mathcal{F})_{\left(\mathfrak{S}_{1} \circ \mathfrak{S}_{2}\right)}^{\mathrm{P}}\left(\mathrm{k}_{1} \mathrm{l}\right)\left(\mathrm{k}_{2} \mathrm{l}\right)=(\mathcal{F})_{\mathfrak{R}_{2}}^{\mathrm{P}}(\mathrm{l}) \vee(\mathcal{F})_{\mathfrak{S}_{1}}^{\mathrm{P}}\left(\mathrm{k}_{1} \mathrm{k}_{2}\right)$

$(\mathcal{T})_{\left(\mathfrak{S}_{1} \circ \mathfrak{S}_{2}\right)}^{\mathrm{N}}\left(\mathrm{k}_{1} \mathrm{l}\right)\left(\mathrm{k}_{2} \mathrm{l}\right)=(\mathcal{T})_{\mathfrak{R}_{2}}^{\mathrm{N}}(\mathrm{l}) \vee(\mathcal{T})_{\mathfrak{S}_{1}}^{\mathrm{N}}\left(\mathrm{k}_{1} \mathrm{k}_{2}\right)$

$(\mathcal{C})_{\left(\mathfrak{S}_{1} \circ \mathfrak{S}_{1}\right)}^{\mathrm{N}}\left(\mathrm{k}_{1} \mathrm{l}\right)\left(\mathrm{k}_{2} \mathrm{l}\right)=(\mathcal{C})_{\mathfrak{R}_{2}}^{\mathrm{N}}(\mathrm{l}) \vee(\mathcal{C})_{\mathfrak{S}_{1}}^{\mathrm{N}}\left(\mathrm{k}_{1} \mathrm{k}_{2}\right)$

$(\mathcal{U})_{\left(\mathfrak{S}_{1} \circ \mathfrak{S}_{2}\right)}^{\mathrm{N}}\left(\mathrm{k}_{1} \mathrm{l}\right)\left(\mathrm{k}_{2} \mathrm{l}\right)=(\mathcal{U})_{\mathfrak{R}_{2}}^{\mathrm{N}}(\mathrm{l}) \wedge(\mathcal{U})_{\mathfrak{S}_{1}}^{\mathrm{N}}\left(\mathrm{k}_{1} \mathrm{k}_{2}\right)$

$(\mathcal{F})_{\left(\mathfrak{S}_{1} \circ \mathfrak{S}_{2}\right)}^{\mathrm{N}}\left(\mathrm{k}_{1} \mathrm{l}\right)\left(\mathrm{k}_{2} \mathrm{l}\right)=(\mathcal{F})_{\mathfrak{R}_{2}}^{\mathrm{N}}(\mathrm{l}) \wedge(\mathcal{F})_{\mathfrak{S}_{1}}^{\mathrm{N}}\left(\mathrm{k}_{1} \mathrm{k}_{2}\right)$,

for all $1 \in \mathfrak{R}_{2}, \mathrm{k}_{1} \mathrm{k}_{2} \in \mathfrak{S}_{1}$.

$(\mathrm{iv})(\mathcal{T})_{\left(\mathfrak{S}_{1} \circ \mathfrak{S}_{2}\right)}^{\mathrm{P}}\left(\mathrm{k}_{1} \mathrm{l}_{1}\right)\left(\mathrm{k}_{2} \mathrm{l}_{2}\right)=(\mathcal{T})_{\mathfrak{S}_{1}}^{\mathrm{P}}\left(\mathrm{k}_{1} \mathrm{k}_{2}\right) \wedge(\mathcal{T})_{\mathfrak{R}_{2}}^{\mathrm{P}}\left(\mathrm{l}_{1}\right) \wedge(\mathcal{T})_{\mathfrak{R}_{2}}^{\mathrm{P}}\left(\mathrm{l}_{2}\right)$

$(\mathcal{C})_{\left(\mathfrak{S}_{1} \circ \mathfrak{S}_{2}\right)}^{\mathrm{P}}\left(\mathrm{k}_{1} \mathrm{l}_{1}\right)\left(\mathrm{k}_{2} \mathrm{l}_{2}\right)=(\mathcal{C})_{\mathfrak{S}_{1}}^{\mathrm{P}}\left(\mathrm{k}_{1} \mathrm{k}_{2}\right) \wedge(\mathcal{C})_{\mathfrak{R}_{2}}^{\mathrm{P}}\left(\mathrm{l}_{1}\right) \wedge(\mathcal{C})_{\mathfrak{R}_{2}}^{\mathrm{P}}\left(\mathrm{l}_{2}\right)$

$(\mathcal{U})_{\left(\mathfrak{S}_{1} \circ \mathfrak{S}_{2}\right)}^{\mathrm{P}}\left(\mathrm{k}_{1} \mathrm{l}_{1}\right)\left(\mathrm{k}_{2} \mathrm{l}_{2}\right)=(\mathcal{U})_{\mathfrak{S}_{1}}^{\mathrm{P}}\left(\mathrm{k}_{1} \mathrm{k}_{2}\right) \vee(\mathcal{U})_{\mathfrak{R}_{2}}^{\mathrm{P}}\left(1_{1}\right) \vee(\mathcal{U})_{\mathfrak{R}_{2}}^{\mathrm{P}}\left(l_{2}\right)$

$(\mathcal{F})_{\left(\mathfrak{S}_{1} \circ \mathfrak{S}_{2}\right)}^{\mathrm{P}}\left(\mathrm{k}_{1} \mathrm{l}_{1}\right)\left(\mathrm{k}_{2} \mathrm{l}_{2}\right)=(\mathcal{F})_{\mathfrak{S}_{1}}^{\mathrm{P}}\left(\mathrm{k}_{1} \mathrm{k}_{2}\right) \vee(\mathcal{F})_{\mathfrak{R}_{2}}^{\mathrm{P}}\left(\mathrm{l}_{1}\right) \vee(\mathcal{F})_{\mathfrak{R}_{2}}^{\mathrm{P}}\left(\mathrm{l}_{2}\right)$

$(\mathcal{T})_{\left(\mathfrak{S}_{1} \circ \mathfrak{S}_{2}\right)}^{\mathrm{N}}\left(\mathrm{k}_{1} \mathrm{l}_{1}\right)\left(\mathrm{k}_{2} \mathrm{l}_{2}\right)=(\mathcal{T})_{\mathfrak{S}_{1}}^{\mathrm{N}}\left(\mathrm{k}_{1} \mathrm{k}_{2}\right) \vee(\mathcal{T})_{\mathfrak{R}_{2}}^{\mathrm{N}}\left(\mathrm{l}_{1}\right) \vee(\mathcal{T})_{\mathfrak{R}_{2}}^{\mathrm{N}}\left(\mathrm{l}_{2}\right)$

$(\mathcal{C})_{\left(\mathfrak{S}_{1} \circ \mathfrak{S}_{2}\right)}^{\mathrm{N}}\left(\mathrm{k}_{1} \mathrm{l}_{1}\right)\left(\mathrm{k}_{2} \mathrm{l}_{2}\right)=(\mathcal{C})_{\mathfrak{S}_{1}}^{\mathrm{N}}\left(\mathrm{k}_{1} \mathrm{k}_{2}\right) \vee(\mathcal{C})_{\mathfrak{R}_{2}}^{\mathrm{N}}\left(\mathrm{l}_{1}\right) \vee(\mathcal{C})_{\mathfrak{R}_{2}}^{\mathrm{N}}\left(\mathrm{l}_{2}\right)$

$(\mathcal{U})_{\left(\mathfrak{S}_{1} \circ \mathfrak{S}_{2}\right)}^{\mathrm{N}}\left(\mathrm{k}_{1} \mathrm{l}_{1}\right)\left(\mathrm{k}_{2} \mathrm{l}_{2}\right)=(\mathcal{U})_{\mathfrak{S}_{1}}^{\mathrm{N}}\left(\mathrm{k}_{1} \mathrm{k}_{2}\right) \wedge(\mathcal{U})_{\mathfrak{R}_{2}}^{\mathrm{N}}\left(l_{1}\right) \wedge(\mathcal{U})_{\mathfrak{R}_{2}}^{\mathrm{N}}\left(l_{2}\right)$

$(\mathcal{F})_{\left(\mathfrak{S}_{1} \circ \mathfrak{S}_{2}\right)}^{\mathrm{N}}\left(\mathrm{k}_{1} \mathrm{l}_{1}\right)\left(\mathrm{k}_{2} \mathrm{l}_{2}\right)=(\mathcal{F})_{\mathfrak{S}_{1}}^{\mathrm{N}}\left(\mathrm{k}_{1} \mathrm{k}_{2}\right) \wedge(\mathcal{F})_{\mathfrak{R}_{2}}^{\mathrm{N}}\left(\mathrm{l}_{1}\right) \wedge(\mathcal{F})_{\mathfrak{R}_{2}}^{\mathrm{N}}\left(1_{2}\right)$,

for all $\mathrm{k}_{1} \mathrm{k}_{2} \in \mathfrak{S}_{1}, 1_{1} \mathrm{l}_{2} \in \mathfrak{S}_{2}$. 
Proposition 3.13 The composition $\mathcal{G}_{1} \circ \mathcal{G}_{2}=\left(\Re_{1} \circ \mathfrak{R}_{2}, \mathfrak{S}_{1} \circ \mathfrak{S}_{2}\right)$ of two QBSVNG, $\mathcal{G}_{1}$ and $\mathcal{G}_{2}$ is also the QBSVNG of $\mathcal{G}_{1} \circ \mathcal{G}_{2}$.

Proof. We divide the proof into three cases:

Case 1: For $\mathrm{k} \in \mathfrak{R}_{1}, \mathrm{l}_{1} \mathrm{l}_{2} \in \mathfrak{S}_{2}$,

$$
\begin{aligned}
& (\mathcal{T})_{\left(\mathfrak{S}_{1} \circ \mathfrak{S}_{2}\right)}^{\mathrm{P}}\left(\left(\mathrm{kl}_{1}\right)\left(\mathrm{kl}_{2}\right)\right)=(\mathcal{T})_{\mathfrak{R}_{1}}^{\mathrm{P}}(\mathrm{k}) \wedge(\mathcal{T})_{\mathfrak{S}_{2}}^{\mathrm{P}}\left(\mathrm{l}_{1} \mathrm{l}_{2}\right) \\
& \leq(\mathcal{T})_{\mathfrak{R}_{1}}^{\mathrm{P}}(\mathrm{k}) \wedge\left[(\mathcal{T})_{\mathfrak{R}_{2}}^{\mathrm{P}}\left(1_{1}\right) \wedge(\mathcal{T})_{\mathfrak{R}_{2}}^{\mathrm{P}}\left(\mathrm{l}_{2}\right)\right] \\
& =\left[(\mathcal{T})_{\mathfrak{R}_{1}}^{\mathrm{P}}(\mathrm{k}) \wedge(\mathcal{T})_{\mathfrak{R}_{2}}^{\mathrm{P}}\left(\mathrm{l}_{1}\right)\right] \wedge\left[(\mathcal{T})_{\mathfrak{R}_{1}}^{\mathrm{P}}(\mathrm{k}) \wedge(\mathcal{T})_{\mathfrak{R}_{2}}^{\mathrm{P}}\left(\mathrm{l}_{2}\right)\right] \\
& =(\mathcal{T})_{\left(\Re_{1} \circ \Re_{2}\right)}^{\mathrm{P}}\left(\mathrm{k}, 1_{1}\right) \wedge(\mathcal{T})_{\left(\Re_{1} \circ \Re_{2}\right)}^{\mathrm{P}}\left(\mathrm{k}, 1_{2}\right) \\
& (\mathcal{C})_{\left(\mathfrak{S}_{1} \circ \mathfrak{S}_{2}\right)}^{\mathrm{P}}\left(\left(\mathrm{kl}_{1}\right)\left(\mathrm{kl}_{2}\right)\right)=(\mathcal{C})_{\mathfrak{R}_{1}}^{\mathrm{P}}(\mathrm{k}) \wedge(\mathcal{C})_{\mathfrak{S}_{2}}^{\mathrm{P}}\left(\mathrm{l}_{1} \mathrm{l}_{2}\right) \\
& \leq(\mathcal{C})_{\mathfrak{R}_{1}}^{\mathrm{P}}(\mathrm{k}) \wedge\left[(\mathcal{C})_{\mathfrak{R}_{2}}^{\mathrm{P}}\left(\mathrm{l}_{1}\right) \wedge(\mathcal{C})_{\mathfrak{R}_{2}}^{\mathrm{P}}\left(\mathrm{l}_{2}\right)\right] \\
& =\left[(\mathcal{C})_{\mathfrak{R}_{1}}^{\mathrm{P}}(\mathrm{k}) \wedge(\mathcal{C})_{\mathfrak{R}_{2}}^{\mathrm{P}}\left(\mathrm{l}_{1}\right)\right] \wedge\left[(\mathcal{C})_{\mathfrak{R}_{1}}^{\mathrm{P}}(\mathrm{k}) \wedge(\mathcal{C})_{\mathfrak{R}_{2}}^{\mathrm{P}}\left(\mathrm{l}_{2}\right)\right] \\
& =(\mathcal{C})_{\left(\Re_{1} \circ \Re_{2}\right)}^{\mathrm{P}}\left(\mathrm{k}, 1_{1}\right) \wedge(\mathcal{C})_{\left(\Re_{1} \circ \Re_{2}\right)}^{\mathrm{P}}\left(\mathrm{k}, \mathrm{l}_{2}\right) \\
& (\mathcal{U})_{\left(\mathfrak{S}_{1} \circ \mathfrak{S}_{2}\right)}^{\mathrm{P}}\left(\left(\mathrm{kl}_{1}\right)\left(\mathrm{kl}_{2}\right)\right)=(\mathcal{U})_{\mathfrak{R}_{1}}^{\mathrm{P}}(\mathrm{k}) \vee(\mathcal{U})_{\mathfrak{S}_{2}}^{\mathrm{P}}\left(1_{1} l_{2}\right) \\
& \leq(\mathcal{U})_{\mathfrak{R}_{1}}^{\mathrm{P}}(\mathrm{k}) \vee\left[(\mathcal{U})_{\mathfrak{R}_{2}}^{\mathrm{P}}\left(l_{1}\right) \vee(\mathcal{U})_{\mathfrak{R}_{2}}^{\mathrm{P}}\left(1_{2}\right)\right] \\
& =\left[(\mathcal{U})_{\mathfrak{R}_{1}}^{\mathrm{P}}(\mathrm{k}) \vee(\mathcal{U})_{\mathfrak{R}_{2}}^{\mathrm{P}}\left(\mathrm{l}_{1}\right)\right] \vee\left[(\mathcal{U})_{\mathfrak{R}_{1}}^{\mathrm{P}}(\mathrm{k}) \vee(\mathcal{U})_{\mathfrak{R}_{2}}^{\mathrm{P}}\left(\mathrm{l}_{2}\right)\right] \\
& =(\mathcal{U})_{\left(\Re_{1} \circ \Re_{2}\right)}^{\mathrm{P}}\left(\mathrm{k}, 1_{1}\right) \vee(\mathcal{U})_{\left(\Re_{1} \circ \Re_{2}\right)}^{\mathrm{P}}\left(\mathrm{k}, \mathrm{l}_{2}\right) \\
& (\mathcal{F})_{\left(\mathfrak{S}_{1} \circ \mathfrak{S}_{2}\right)}^{\mathrm{P}}\left(\left(\mathrm{kl}_{1}\right)\left(\mathrm{kl}_{2}\right)\right)=(\mathcal{F})_{\mathfrak{R}_{1}}^{\mathrm{P}}(\mathrm{k}) \vee(\mathcal{F})_{\mathfrak{S}_{2}}^{\mathrm{P}}\left(\mathrm{l}_{1} \mathrm{l}_{2}\right) \\
& \leq(\mathcal{F})_{\mathfrak{R}_{1}}^{\mathrm{P}}(\mathrm{k}) \vee\left[(\mathcal{F})_{\mathfrak{R}_{2}}^{\mathrm{P}}\left(1_{1}\right) \vee(\mathcal{F})_{\mathfrak{R}_{2}}^{\mathrm{P}}\left(\mathrm{l}_{2}\right)\right] \\
& =\left[(\mathcal{F})_{\mathfrak{R}_{1}}^{\mathrm{P}}(\mathrm{k}) \vee(\mathcal{F})_{\mathfrak{R}_{2}}^{\mathrm{P}}\left(\mathrm{l}_{1}\right)\right] \vee\left[(\mathcal{F})_{\mathfrak{R}_{1}}^{\mathrm{P}}(\mathrm{k}) \vee(\mathcal{F})_{\mathfrak{R}_{2}}^{\mathrm{P}}\left(\mathrm{l}_{2}\right)\right] \\
& =(\mathcal{F})_{\left(\Re_{1} \circ \Re_{2}\right)}^{\mathrm{P}}\left(\mathrm{k}, 1_{1}\right) \vee(\mathcal{F})_{\left(\Re_{1} \circ \Re_{2}\right)}^{\mathrm{P}}\left(\mathrm{k}, 1_{2}\right),
\end{aligned}
$$

for all $\left(\mathrm{kl}_{1}, \mathrm{kl}_{2}\right) \in \mathfrak{R}_{1} \circ \mathfrak{R}_{2}$.

Case 2: for $\mathrm{k} \in \mathfrak{R}_{2}, 1_{1} \mathrm{l}_{2} \in \mathfrak{S}_{1}$.

$$
\begin{aligned}
& (\mathcal{T})_{\left(\mathfrak{S}_{1} \circ \mathfrak{S}_{2}\right)}^{\mathrm{P}}\left(\left(1_{1} \mathrm{k}\right)\left(1_{2} \mathrm{k}\right)\right)=(\mathcal{T})_{\mathfrak{R}_{2}}^{\mathrm{P}}(\mathrm{k}) \wedge(\mathcal{T})_{\mathfrak{S}_{1}}^{\mathrm{P}}\left(1_{1} 1_{2}\right) \\
& \leq(\mathcal{T})_{\mathfrak{R}_{2}}^{\mathrm{P}}(\mathrm{k}) \wedge\left[(\mathcal{T})_{\mathfrak{R}_{1}}^{\mathrm{P}}\left(\mathrm{l}_{1}\right) \wedge(\mathcal{T})_{\mathfrak{R}_{1}}^{\mathrm{P}}\left(l_{2}\right)\right] \\
& =\left[(\mathcal{T})_{\mathfrak{R}_{2}}^{\mathrm{P}}(\mathrm{k}) \wedge(\mathcal{T})_{\mathfrak{R}_{2}}^{\mathrm{P}}\left(1_{1}\right)\right] \wedge\left[(\mathcal{T})_{\mathfrak{R}_{2}}^{\mathrm{P}}(\mathrm{k}) \wedge(\mathcal{T})_{\mathfrak{R}_{1}}^{\mathrm{P}}\left(l_{2}\right)\right] \\
& =(\mathcal{T})_{\left(\mathfrak{R}_{1} \circ \mathfrak{R}_{2}\right)}^{\mathrm{P}}\left(1_{1}, \mathrm{k}\right) \wedge(\mathcal{T})_{\left(\Re_{1} \circ \mathfrak{R}_{2}\right)}^{\mathrm{P}}\left(\mathrm{l}_{2}, \mathrm{k}\right) \\
& (\mathcal{C})_{\left(\mathfrak{S}_{1} \circ \mathfrak{S}_{2}\right)}^{\mathrm{P}}\left(\left(1_{1} \mathrm{k}\right)\left(1_{2} \mathrm{k}\right)\right)=(\mathcal{C})_{\mathfrak{R}_{2}}^{\mathrm{P}}(\mathrm{k}) \wedge(\mathcal{C})_{\mathfrak{S}_{1}}^{\mathrm{P}}\left(1_{1} 1_{2}\right) \\
& \leq(\mathcal{C})_{\mathfrak{R}_{2}}^{\mathrm{P}}(\mathrm{k}) \wedge\left[(\mathcal{C})_{\mathfrak{R}_{1}}^{\mathrm{P}}\left(1_{1}\right) \wedge(\mathcal{C})_{\mathfrak{R}_{1}}^{\mathrm{P}}\left(\mathrm{l}_{2}\right)\right]
\end{aligned}
$$




$$
\begin{aligned}
& =\left[(\mathcal{C})_{\mathfrak{R}_{2}}^{\mathrm{P}}(\mathrm{k}) \wedge(\mathcal{C})_{\mathfrak{R}_{2}}^{\mathrm{P}}\left(\mathrm{l}_{1}\right)\right] \wedge\left[(\mathcal{C})_{\mathfrak{R}_{2}}^{\mathrm{P}}(\mathrm{k}) \wedge(\mathcal{C})_{\mathfrak{R}_{1}}^{\mathrm{P}}\left(\mathrm{l}_{2}\right)\right] \\
& =(\mathcal{C})_{\left(\Re_{1} \circ \Re_{2}\right)}^{\mathrm{P}}\left(1_{1}, \mathrm{k}\right) \wedge(\mathcal{C})_{\left(\Re_{1} \circ \Re_{2}\right)}^{\mathrm{P}}\left(\mathrm{l}_{2}, \mathrm{k}\right) \\
& (\mathcal{U})_{\left(\mathfrak{S}_{1} \circ \mathfrak{S}_{2}\right)}^{\mathrm{P}}\left(\left(1_{1} \mathrm{k}\right)\left(\mathrm{l}_{2} \mathrm{k}\right)\right)=(\mathcal{U})_{\mathfrak{R}_{2}}^{\mathrm{P}}(\mathrm{k}) \vee(\mathcal{U})_{\mathfrak{S}_{1}}^{\mathrm{P}}\left(1_{1} 1_{2}\right) \\
& \leq(\mathcal{U})_{\mathfrak{R}_{2}}^{\mathrm{P}}(\mathrm{k}) \vee\left[(\mathcal{U})_{\mathfrak{R}_{1}}^{\mathrm{P}}\left(l_{1}\right) \vee(\mathcal{U})_{\mathfrak{R}_{1}}^{\mathrm{P}}\left(l_{2}\right)\right] \\
& =\left[(\mathcal{U})_{\mathfrak{R}_{2}}^{\mathrm{P}}(\mathrm{k}) \vee(\mathcal{U})_{\mathfrak{R}_{2}}^{\mathrm{P}}\left(\mathrm{l}_{1}\right)\right] \vee\left[(\mathcal{U})_{\mathfrak{R}_{2}}^{\mathrm{P}}(\mathrm{k}) \vee(\mathcal{U})_{\mathfrak{R}_{1}}^{\mathrm{P}}\left(1_{2}\right)\right] \\
& =(\mathcal{U})_{\left(\Re_{1} \circ \Re_{2}\right)}^{\mathrm{P}}\left(l_{1}, \mathrm{k}\right) \vee(\mathcal{U})_{\left(\Re_{1} \circ \Re_{2}\right)}^{\mathrm{P}}\left(\mathrm{l}_{2}, \mathrm{k}\right) \\
& (\mathcal{F})_{\left(\mathfrak{S}_{1} \circ \mathfrak{S}_{2}\right)}^{\mathrm{P}}\left(\left(\mathrm{l}_{1} \mathrm{k}\right)\left(\mathrm{l}_{2} \mathrm{k}\right)\right)=(\mathcal{F})_{\mathfrak{R}_{2}}^{\mathrm{P}}(\mathrm{k}) \vee(\mathcal{F})_{\mathfrak{S}_{1}}^{\mathrm{P}}\left(l_{1} \mathrm{l}_{2}\right) \\
& \leq(\mathcal{F})_{\mathfrak{R}_{2}}^{\mathrm{P}}(\mathrm{k}) \vee\left[(\mathcal{F})_{\mathfrak{R}_{1}}^{\mathrm{P}}\left(1_{1}\right) \vee(\mathcal{F})_{\mathfrak{R}_{1}}^{\mathrm{P}}\left(1_{2}\right)\right] \\
& =\left[(\mathcal{F})_{\mathfrak{R}_{2}}^{\mathrm{P}}(\mathrm{k}) \vee(\mathcal{F})_{\mathfrak{R}_{2}}^{\mathrm{P}}\left(\mathrm{l}_{1}\right)\right] \vee\left[(\mathcal{F})_{\mathfrak{R}_{2}}^{\mathrm{P}}(\mathrm{k}) \vee(\mathcal{F})_{\mathfrak{R}_{1}}^{\mathrm{P}}\left(1_{2}\right)\right] \\
& =(\mathcal{F})_{\left(\Re_{1} \circ \Re_{2}\right)}^{\mathrm{P}}\left(\mathrm{l}_{1}, \mathrm{k}\right) \vee(\mathcal{F})_{\left(\Re_{1} \circ \Re_{2}\right)}^{\mathrm{P}}\left(\mathrm{l}_{2}, \mathrm{k}\right),
\end{aligned}
$$

for all $\left(1_{1} \mathrm{k}, 1_{2} \mathrm{k}\right) \in \mathcal{G}_{1} \circ \mathcal{G}_{2}$.

Case 3: For all $\mathrm{k}_{1} \mathrm{l}_{1} \in \mathfrak{S}_{1}, \mathrm{k}_{2} \mathrm{l}_{2} \in \mathfrak{S}_{2} \mathrm{l}_{1} \neq \mathrm{l}_{2}$,

$$
\begin{aligned}
& (\mathcal{T})_{\left(\mathfrak{S}_{1} \circ \mathfrak{S}_{2}\right)}^{\mathrm{P}}\left(\left(\mathrm{k}_{1} \mathrm{l}_{1}\right)\left(\mathrm{k}_{2} \mathrm{l}_{2}\right)\right)=(\mathcal{T})_{\mathfrak{S}_{1}}^{\mathrm{P}}\left(\mathrm{k}_{1} \mathrm{k}_{2}\right) \wedge(\mathcal{T})_{\mathfrak{R}_{2}}^{\mathrm{P}}\left(\mathrm{l}_{1}\right) \wedge(\mathcal{T})_{\mathfrak{R}_{2}}^{\mathrm{P}}\left(\mathrm{l}_{2}\right) \\
& \leq\left[(\mathcal{T})_{\mathfrak{R}_{1}}^{\mathrm{P}}\left(\mathrm{k}_{1}\right) \wedge(\mathcal{T})_{\mathfrak{R}_{1}}^{\mathrm{P}}\left(\mathrm{k}_{2}\right)\right] \wedge\left[(\mathcal{T})_{\mathfrak{R}_{2}}^{\mathrm{P}}\left(\mathrm{l}_{1}\right) \wedge(\mathcal{T})_{\mathfrak{R}_{2}}^{\mathrm{P}}\left(\mathrm{l}_{2}\right)\right] \\
& =\left[(\mathcal{T})_{\mathfrak{R}_{1}}^{\mathrm{P}}\left(\mathrm{k}_{1}\right) \wedge(\mathcal{T})_{\mathfrak{R}_{2}}^{\mathrm{P}}\left(\mathrm{l}_{1}\right)\right] \wedge\left[(\mathcal{T})_{\mathfrak{R}_{1}}^{\mathrm{P}}\left(\mathrm{k}_{2}\right) \wedge(\mathcal{T})_{\mathfrak{R}_{2}}^{\mathrm{P}}\left(\mathrm{l}_{2}\right)\right] \\
& =(\mathcal{T})_{\left(\Re_{1} \circ \Re_{2}\right)}^{\mathrm{P}}\left(\mathrm{k}_{1}, 1_{1}\right) \wedge(\mathcal{T})_{\left(\Re_{1} \circ \Re_{2}\right)}^{\mathrm{P}}\left(\mathrm{k}_{2}, 1_{2}\right) \\
& (\mathcal{C})_{\left(\mathfrak{S}_{1} \circ \mathfrak{S}_{2}\right)}^{\mathrm{P}}\left(\left(\mathrm{k}_{1} \mathrm{l}_{1}\right)\left(\mathrm{k}_{2} \mathrm{l}_{2}\right)\right)=(\mathcal{C})_{\mathfrak{S}_{1}}^{\mathrm{P}}\left(\mathrm{k}_{1} \mathrm{k}_{2}\right) \wedge(\mathcal{C})_{\mathfrak{R}_{2}}^{\mathrm{P}}\left(\mathrm{l}_{1}\right) \wedge(\mathcal{C})_{\mathfrak{R}_{2}}^{\mathrm{P}}\left(\mathrm{l}_{2}\right) \\
& \leq\left[(\mathcal{C})_{\mathfrak{R}_{1}}^{\mathrm{P}}\left(\mathrm{k}_{1}\right) \wedge(\mathcal{C})_{\mathfrak{R}_{1}}^{\mathrm{P}}\left(\mathrm{k}_{2}\right)\right] \wedge\left[(\mathcal{C})_{\mathfrak{R}_{2}}^{\mathrm{P}}\left(\mathrm{l}_{1}\right) \wedge(\mathcal{C})_{\mathfrak{R}_{2}}^{\mathrm{P}}\left(\mathrm{l}_{2}\right)\right] \\
& =\left[(\mathcal{C})_{\mathfrak{R}_{1}}^{\mathrm{P}}\left(\mathrm{k}_{1}\right) \wedge(\mathcal{C})_{\mathfrak{R}_{2}}^{\mathrm{P}}\left(\mathrm{l}_{1}\right)\right] \wedge\left[(\mathcal{C})_{\mathfrak{R}_{1}}^{\mathrm{P}}\left(\mathrm{k}_{2}\right) \wedge(\mathcal{C})_{\mathfrak{R}_{2}}^{\mathrm{P}}\left(\mathrm{l}_{2}\right)\right] \\
& =(\mathcal{C})_{\left(\Re_{1} \circ \Re_{2}\right)}^{\mathrm{P}}\left(\mathrm{k}_{1}, 1_{1}\right) \wedge(\mathcal{C})_{\left(\Re_{1} \circ \Re_{2}\right)}^{\mathrm{P}}\left(\mathrm{k}_{2}, 1_{2}\right) \\
& (\mathcal{U})_{\left(\mathfrak{S}_{1} \circ \mathfrak{S}_{2}\right)}^{\mathrm{P}}\left(\left(\mathrm{k}_{1} \mathrm{l}_{1}\right)\left(\mathrm{k}_{2} \mathrm{l}_{2}\right)\right)=(\mathcal{U})_{\mathfrak{S}_{1}}^{\mathrm{P}}\left(\mathrm{k}_{1} \mathrm{k}_{2}\right) \vee(\mathcal{U})_{\mathfrak{R}_{2}}^{\mathrm{P}}\left(\mathrm{l}_{1}\right) \vee(\mathcal{U})_{\mathfrak{R}_{2}}^{\mathrm{P}}\left(\mathrm{l}_{2}\right) \\
& \leq\left[(\mathcal{U})_{\mathfrak{R}_{1}}^{\mathrm{P}}\left(\mathrm{k}_{1}\right) \vee(\mathcal{U})_{\mathfrak{R}_{1}}^{\mathrm{P}}\left(\mathrm{k}_{2}\right)\right] \vee\left[(\mathcal{U})_{\mathfrak{R}_{2}}^{\mathrm{P}}\left(1_{1}\right) \vee(\mathcal{U})_{\mathfrak{R}_{2}}^{\mathrm{P}}\left(\mathrm{l}_{2}\right)\right] \\
& =\left[(\mathcal{U})_{\mathfrak{R}_{1}}^{\mathrm{P}}\left(\mathrm{k}_{1}\right) \vee(\mathcal{U})_{\mathfrak{R}_{2}}^{\mathrm{P}}\left(l_{1}\right)\right] \vee\left[(\mathcal{U})_{\mathfrak{R}_{1}}^{\mathrm{P}}\left(\mathrm{k}_{2}\right) \vee(\mathcal{U})_{\mathfrak{R}_{2}}^{\mathrm{P}}\left(\mathrm{l}_{2}\right)\right] \\
& =(\mathcal{U})_{\left(\Re_{1} \circ \Re_{2}\right)}^{\mathrm{P}}\left(\mathrm{k}_{1}, 1_{1}\right) \vee(\mathcal{U})_{\left(\Re_{1} \circ \Re_{2}\right)}^{\mathrm{P}}\left(\mathrm{k}_{2}, 1_{2}\right) \\
& (\mathcal{F})_{\left(\mathfrak{S}_{1} \circ \mathfrak{S}_{2}\right)}^{\mathrm{P}}\left(\left(\mathrm{k}_{1} \mathrm{l}_{1}\right)\left(\mathrm{k}_{2} \mathrm{l}_{2}\right)\right)=(\mathcal{F})_{\mathfrak{S}_{1}}^{\mathrm{P}}\left(\mathrm{k}_{1} \mathrm{k}_{2}\right) \vee(\mathcal{F})_{\mathfrak{R}_{2}}^{\mathrm{P}}\left(\mathrm{l}_{1}\right) \vee(\mathcal{F})_{\mathfrak{R}_{2}}^{\mathrm{P}}\left(\mathrm{l}_{2}\right) \\
& \leq\left[(\mathcal{F})_{\mathfrak{R}_{1}}^{\mathrm{P}}\left(\mathrm{k}_{1}\right) \vee(\mathcal{F})_{\mathfrak{R}_{1}}^{\mathrm{P}}\left(\mathrm{k}_{2}\right)\right] \vee\left[(\mathcal{F})_{\mathfrak{R}_{2}}^{\mathrm{P}}\left(\mathrm{l}_{1}\right) \vee(\mathcal{F})_{\mathfrak{R}_{2}}^{\mathrm{P}}\left(\mathrm{l}_{2}\right)\right] \\
& =\left[(\mathcal{F})_{\mathfrak{R}_{1}}^{\mathrm{P}}\left(\mathrm{k}_{1}\right) \vee(\mathcal{F})_{\mathfrak{R}_{2}}^{\mathrm{P}}\left(\mathrm{l}_{1}\right)\right] \vee\left[(\mathcal{F})_{\mathfrak{R}_{1}}^{\mathrm{P}}\left(\mathrm{k}_{2}\right) \vee(\mathcal{F})_{\mathfrak{R}_{2}}^{\mathrm{P}}\left(\mathrm{l}_{2}\right)\right]
\end{aligned}
$$


$=(\mathcal{F})_{\left(\Re_{1} \circ \Re_{2}\right)}^{\mathrm{P}}\left(\mathrm{k}_{1}, 1_{1}\right) \vee(\mathcal{F})_{\left(\Re_{1} \circ \Re_{2}\right)}^{\mathrm{P}}\left(\mathrm{k}_{2}, 1_{2}\right)$,

for all $\left(\mathrm{k}_{1} \mathrm{l}_{1}, \mathrm{k}_{2} \mathrm{l}_{2}\right) \in \mathfrak{R}_{1} \circ \mathfrak{R}_{2}$. Similarly, one can prove the negative part. This completes the proof.

\section{Conclusion}

This manuscript dealt with the new concept of quadripartitioned bipolar single valued neutrosophic graph. The Cartesian product, cross product, lexicographic product, strong product and composition of QBSVNGs are discussed. The proposed concepts are illustrated with examples. The proposed sets, graphs, and operations offer sufficient capability to overcome the related reliance on imprecise data. In future, one can study the developed concepts in the quadripartitioned neutrosophic soft graphs, interval valued quadripartitioned neutrosophic graphs, etc. Also, one can extend the developed concepts into isomorphic properties and regularity properties in the proposed graph structures.

Acknowledgement: The authors are grateful to the anonymous referees for a careful checking of the details and for helpful comments that improved this paper.

Funding Statement: This work was supported by the Taif University Researchers Supporting Project (TURSP-2020/246), Taif University, Taif, Saudi Arabia.

Conflicts of Interest: The authors declare that they have no conflict of interest.

\section{References}

1. Lee, K. M. (2000). Bipolar-valued fuzzy sets and their operations. Proceedings International Conference on Intelligent Technologies, pp. 307-312. Bangkok, Thailand.

2. Mahmood, T. (2020). A novel approach towards bipolar soft sets and their applications. Journal of Mathematics, 2020(5), 1-11. DOI 10.1155/2020/4690808.

3. Akram, M., Sitara, M. (2017). Bipolar neutrosophic graph structures. Journal of the Indonesian Mathematical Society, 23(1), 55-80. DOI 10.22342/jims.23.1.442.55-80.

4. Akram, M. (2011). Bipolar fuzzy graphs. Information Sciences, 181(24), 5548-5564. DOI 10.1016/j.ins. 2011.07.037.

5. Wang, H., Smarandache, F., Zhang, Y., Sunderraman, R. (2010). Single-valued neutrosophic sets. Multispace and Multistructure, 4, 410-413.

6. Smarandache, F. (1999). A unifying field in logics: Neutrosophic logic. In Philosophy. USA: American Research Press.

7. Bhattacharya, S. (2005). Neutrosophic informationfusion applied to the options market. Investment Management and Financial 855 Innovations, 1, 139-145.

8. Aggarwal, S., Biswas, R., Ansari, A. Q. (2010). Neutrosophic modeling and control. International Conference on Computer and Communication Technology, pp. 718-723. Switzerland: ETH Zurich.

9. Guo, Y., Cheng, H. D. (2009). New neutrosophic approach to image segmentation. Pattern Recognition, 42(5), 587-595. DOI 10.1016/j.patcog.2008.10.002.

10. Ye, J., Fu, J. (2016). Multi-period medical diagnosis method using a single valued neutrosophic similarity measure based on tangent function. Computer Methods and Programs in Biomedicine, 123(2), 142-149. DOI 10.1016/j.cmpb.2015.10.002.

11. Ye, J. (2013). Multicriteria decision-making method using the correlation coefficient under single valued neutrosophic environment. International Journal of General Systems, 42(4), 386-394. DOI 10.1080/03081079.2012.761609.

12. Jana, C., Pal, M. (2019). A robust single-valued neutrosophic soft aggregation operators in multi-criteria decision making. Symmetry, 11(1), 110. DOI 10.3390/sym11010110. 
13. Akram, M., Shahzadi, S. (2017). Neutrosophic soft graphs with application. Journal of Intelligent \& Fuzzy Systems, 32(1), 841-858. DOI 10.3233/JIFS-16090.

14. Ali, M., Smarandache, F. (2017). Complex neutrosophic set. Neural Computing \& Application, 28(7), 18171834. DOI 10.1007/s00521-015-2154-y.

15. Hussain, S. S., Hussain, R. J., Jun, Y. B., Smarandache, F. (2019). Neutrosophic bipolar vague set and its application to neutrosophic bipolar vague graphs. Neutrosophic Sets and Systems, 28, 69-86. DOI 10.5281/zenodo.3387802.

16. Hussain, S. S., Hussain, R. J., Smarandache, F. (2019). On neutrosophic vague graphs. Neutrosophic Sets and Systems, 28, 245-258. DOI 10.5281/zenodo.3382550.

17. Hussain, S. S., Broumi, S., Jun, Y. B., Durga, N. (2019). Intuitionistic bipolar neutrosophic set and its application to intuitionistic bipolar neutrosophic graphs. Annals of Communication in Mathematics, 2(2), 121-140.

18. Hussain, S. S., Hussain, R. J., Smarandache, F. (2019). Domination number in neutrosophic soft graphs. Neutrosophic Sets and Systems, 28(1), 228-244. DOI 10.5281/zenodo.3382548.

19. Liang, R. X., Wang, J. Q., Li, L. (2018). Multi-criteria group decision-making method based on interdependent inputs of single-valued trapezoidal neutrosophic information. Neural Computing and Application, 30(1), 241-260. DOI 10.1007/s00521-016-2672-2.

20. Sahin, R., Liu, P. (2017). Correlation coefficient of single-valued neutrosophic hesitant fuzzy sets and its applications in decision making. Neural Computing and Application, 28(6), 1387-1395. DOI 10.1007/s00521-015-2163-x.

21. Chatterjee, R., Majumdar, P., Samanta, S. K. (2016). On some similarity measures and entropy on quadripartitioned single valued neutrosophic sets. Journal of Intelligent \& Fuzzy Systems, 30(4), 2475-2485. DOI 10.3233/IFS-152017.

22. Belnap, N. D. (1977). A useful four-valued logic. Modern uses of multiple-valued logic. Dordrecht: Springer.

23. Smarandache, F. (2013). n-valued refined neutrosophic logic and its applications to physics. Progress in Physics, 4, 143-146. DOI 10.5281/zenodo.49149.

24. Akram, M., Shahzadi, G. (2017). Operations on single-valued neutrosophic graphs. Journal of Uncertain Systems, 11(1), 1-26.

25. Sinha, K., Majumdar, P. (2020). Bipolar quadripartitioned single valued neutrosophic sets. Proyecciones (Antofagasta), 39(6), 1597-1614. DOI 10.22199/issn.0717-6279-2020-06-0095.

26. Akram, M., Shahzadi, G. (2019). Bipolar neutrosophic graphs. In: Kahraman, C., Tay, O. (Eds.), Fuzzy multi-criteria decision-making using neutrosophic sets. Studies in fuzziness and soft computing, pp. 382-394, Cham: Springer.

27. Broumi, S., Talea, M., Bakali, A., Smarandache, F. (2016). On bipolar single valued neutrosophic graphs. Journal of New Theory, (11), 84-102.

28. Muhiuddin, G. (2021). p-ideals of BCI-algebras based on neutrosophic N-structures. Journal of Intelligent \& Fuzzy Systems, 40(1), 1097-1105. DOI 10.3233/JIFS-201309.

29. Hussain, S. S., Hussain, R. J., Muhiuddin, G. (2020). Neutrosophic vague line graphs. Neutrosophic Sets and Systems, 36, 121-130. DOI 10.5281/zenodo.4065406.

30. Muhiuddin, G., Al-Kenani, A. N., Roh, E. H., Jun, Y. B. (2019). Implicative neutrosophic quadruple BCKalgebras and ideals. Symmetry, 11(2), 277. DOI 10.3390/sym11020277.

31. Muhiuddin, G., Kim, S. J., Jun, Y. B. (2019). Implicative N-ideals of BCK-algebras based on neutrosophic N-structures. Discrete Mathematics, Algorithms and Applications, 11(1), 1950011. DOI 10.1142/S1793830919500113.

32. Roy, S., Lee, J. G., Pal, A., Samanta, S. K. (2020). Similarity measures of quadripartitioned single valued bipolar neutrosophic sets and its application in multi-criteria decision making problems. Symmetry, 12(6), 1012. DOI 10.3390/sym12061012. 